\title{
ICES REPORT 10-06
}

March 2010

\section{Mixed hp-Finite Element Method for Linear Elasticity with Weakly Imposed Symmetry. II. Curvilinear Elements in 2D \\ by \\ Weifeng Qiu and Leszek Demkowicz}

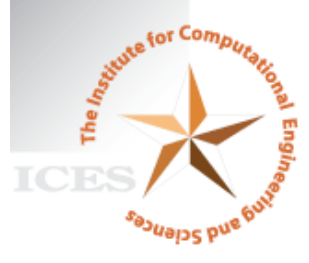

The Institute for Computational Engineering and Sciences The University of Texas at Austin 


\title{
MIXED $h p$-FINITE ELEMENT METHOD FOR LINEAR ELASTICITY WITH WEAKLY IMPOSED SYMMETRY. II. CURVILINEAR ELEMENTS IN 2D
}

\author{
WEIFENG QIU AND LESZEK DEMKOWICZ
}

\begin{abstract}
We continue our study on variable order Arnold-Falk-Winther elements for 2D elasticity in context of parametric curvilinear elements. We present an asymptotic stability result and illustrate it with numerical experiments.
\end{abstract}

\section{INTRODUCTION}

1.1. Elasticity problem. Linear elasticity is a classical subject, and it has been studied for a long time. The presented research is motivated with a class of timeharmonic problems formulated as follows. Let $\Omega \subset \mathbb{R}^{n}, n=2,3$, denote a bounded domain occupied by the elastic body. Assume that the boundary of $\Omega, \Gamma=\partial \Omega$ has been split into two disjoint subsets $\Gamma_{1}$ and $\Gamma_{2}$,

$$
\Gamma=\overline{\Gamma_{1}} \cup \overline{\Gamma_{2}}, \quad \Gamma_{1} \cap \Gamma_{2}=\emptyset
$$

Subsets $\Gamma_{1}, \Gamma_{2}$ are assumed to be (relatively) open in $\Gamma$. We seek:

- displacement vector $u_{i}(x), x \in \Omega$,

- linearized strain tensor $\epsilon_{i j}(x), x \in \Omega$, and

- stress tensor $\sigma_{i j}(x), x \in \Omega$

that satisfy the following system of equations and boundary conditions.

- Cauchy's geometrical relation between the displacement and strain,

$$
\epsilon_{i j}=\frac{1}{2}\left(u_{i, j}+u_{j, i}\right), \quad x \in \Omega
$$

- Equations of motion resulting from the principle of linear momentum,

$$
-\sigma_{i j, j}-\rho(x) \omega^{2} u_{i}=f_{i}(x), \quad x \in \Omega
$$

- Symmetry of the stress tensor being a consequence of the principle of angular momentum,

$$
\sigma_{i j}=\sigma_{j i}, \quad x \in \Omega
$$

- Constitutive equations for linear elasticity,

$$
\sigma_{i j}=E_{i j k l}(x) \epsilon_{k l}, \quad x \in \Omega
$$

- Kinematic boundary conditions,

$$
u_{i}=u_{i}^{0}, \quad x \in \Gamma_{1}
$$

2000 Mathematics Subject Classification. 65N30, 65N12.

Key words and phrases. plane elasticity, mixed formulation, $h p$ elements, curvilinear meshes. 
- Traction boundary conditions,

$$
\sigma_{i j} n_{j}=g_{i}(x), \quad x \in \Gamma_{2}
$$

Here:

- $\rho$ is the density of the body,

- $f_{i}$ are volume forces prescribed within the body,

- $g_{i}$ are tractions prescribed on $\Gamma_{2}$ part of the boundary,

- $u_{i}^{0}$ are displacements prescribed on $\Gamma_{1}$ part of the boundary,

- $n_{j}$ is the unit outward normal vector for boundary $\Gamma$, and

- $E_{i j k l}$ is the tensor of elasticities.

As usual, commas denote partial derivatives, and we use the Einstein's summation convention. Vector $t_{i}:=\sigma_{i j} n_{j}$ is known as the traction or stress vector.

The geometrical relations imply the symmetry of the strain tensor. The symmetry of strain and stress tensors imply then the usual (minor) symmetry conditions for the elasticities,

$$
E_{i j k l}=E_{j i k l}=E_{i j l k}
$$

The laws of thermodynamics imply an additional (major) symmetry condition,

$$
E_{i j k l}=E_{k l i j}
$$

and positive definiteness condition,

$$
E_{i j k l} \xi_{i j} \xi_{k l}>0, \quad \forall \xi_{i j}=\xi_{i j}
$$

The last condition implies that the constitutive equation can be inverted to represent strains in terms of stresses,

$$
\epsilon_{k l}=C_{k l i j} \sigma_{i j}
$$

where $C_{k l i j}=E_{i j k l}^{-1}$ is known as the compliance tensor and satisfies analogous symmetry and positive-definiteness properties.

For isotropic materials, the elasticities tensor is expressed in terms of two independent Lame's constants $\mu$ and $\lambda$,

$$
E_{i j k l}=\mu\left(\delta_{i k} \delta_{j l}+\delta_{i l} \delta_{j k}\right)+\lambda \delta_{i j} \delta_{k l}
$$

and the constitutive equation reduces to,

$$
\sigma_{i j}=2 \mu \epsilon_{i j}+\lambda \epsilon_{k k} \delta_{i j}
$$

where $\delta_{i j}$ denotes the Kronecker's delta. Inverting, we obtain

$$
\epsilon_{i j}=A \sigma_{i j}, \quad \epsilon_{i j}=\frac{1}{2 \mu} \sigma_{i j}-\frac{\lambda}{2 \mu(2 \mu+n \lambda)} \sigma_{k k} \delta_{i j}
$$

Of particular interest is the case of nearly incompressible material corresponding to $\lambda \rightarrow \infty$. Notice that the norm of the elasticities blows up then to infinity but the norm of the compliance tensor remains uniformly bounded. This suggests that formulations based on the compliance relation have a chance to remain uniformly stable for nearly incompressible materials. Another reason of using formulations based on compliance relation is for (visco)elastic vibration problems for structures with large material contrast. For a more detailed discussion on these problems, we refer to the introduction in [19].

\subsection{Variational formulations for elasticity.}


Dual-Mixed Formulation. We eliminate the strain tensor and combine geometrical relations (1.1) and constitutive equation (1.4) in a single equation,

$$
C_{k l i j} \sigma_{i j}=\frac{1}{2}\left(u_{k, l}+u_{l, k}\right)
$$

Classical formulation for elasticity is based on satisfying the equation above in a strong sense while relaxing the momentum equations. The idea behind the dualmixed formulation for elasticity is exactly opposite, the equation above is relaxed whereas momentum equations (1.2) are satisfied in a strong sense.

The final formulation includes building in kinematic boundary conditions and it reads as follows.

$$
\left\{\begin{array}{l}
\sigma \in H(\operatorname{div}, \Omega, \mathbb{S}): \sigma_{i j} n_{j} \quad=g_{i} \text { on } \Gamma_{2}, u \in L^{2}(\Omega, \mathbb{V}) \\
\int_{\Omega} C_{i j k l} \sigma_{k l} \tau_{i j}+\int_{\Omega} u_{i} \tau_{i j, j}=\int_{\Gamma_{1}} u_{i}^{0} \tau_{i j} n_{j} \quad \forall \tau \in H(\operatorname{div}, \Omega, \mathbb{S}): \tau_{i j} n_{j}=0 \text { on } \Gamma_{2} \\
-\int_{\Omega} \sigma_{i j, j} v_{i}-\omega^{2} \int_{\Omega} \rho u_{i} v_{i}=\int_{\Omega} f_{i} v_{i} \quad \forall v \in L^{2}(\Omega, \mathbb{V})
\end{array}\right.
$$

Above, $\mathbb{V}=\mathbb{R}^{3}$ and $\mathbb{S}$ denotes the space of symmetric tensor. $L^{2}(\Omega, \mathbb{V})$ denotes the space of square integrable functions with values in $\mathbb{V}$, and $H(\operatorname{div}, \Omega, \mathbb{S})$ denotes the space of square-integrable functions with values in $\mathbb{S}$, whose row-wise divergence lives in $L^{2}(\Omega, \mathbb{V})$. The traction conditions are satisfied in the sense of traces for functions from $H(\operatorname{div}, \Omega)$ (they live in $H^{-1 / 2}(\Gamma)$ ).

The corresponding static case can be derived formally by considering the socalled Hellinger-Reissner functional, and the variational formulation is frequently identified as the Hellinger-Reissner variational principle.

Dual-Mixed Formulation with Weakly Imposed Symmetry. The symmetry condition is difficult to enforce on the discrete level. This has led to the idea of relaxing the symmetric function and satisfying it in a weaker, integral form. This is obtained by introducing tensor-valued test functions $q$ with values in the space of antisymmetric tensors $\mathbb{K}:=\left\{q_{i j}: q_{i j}=-q_{j i}\right\}$, and replacing the symmetry condition with an integral condition,

$$
\int_{\Omega} \sigma_{i j} q_{i j}=0, \quad \forall q \in L^{2}(\Omega, \mathbb{K})
$$

On the continuous level, the integral condition implies the pointwise condition (understood in the $L^{2}$ sense), but on the discrete level, with an appropriate choice of spaces, the integral condition does not necessary imply the symmetry condition pointwise.

The extra condition leads to an extra unknown. The derivation of the weak form of the constitutive equation has to be revisited. We start by introducing the tensor of infinitesimal rotations,

$$
p_{i j}=\frac{1}{2}\left(u_{i, j}-u_{j, i}\right)
$$

Upon eliminating the strain tensor, the constitutive equation in the compliance form is now rewritten as,

$$
C_{i j k l} \sigma_{k l}=\frac{1}{2}\left(u_{i, j}+u_{j, i}\right)=u_{i, j}-p_{i j}
$$


Multiplication with a test function $\tau$ (now, not necessarily symmetric), integration over $\Omega$, and integration by parts, leads to a new relaxed version of the equation,

$$
\int_{\Omega} C_{i j k l} \sigma_{k l} \tau_{i j}=-\int_{\Omega} u_{i} \tau_{i j, j}+\int_{\Gamma} u_{i} \tau_{i j} n_{j}-\int_{\Omega} p_{i j} \tau_{i j}
$$

We obtain a new variational formulation in the form:

(1.6)

$$
\left\{\begin{array}{l}
\sigma \in H(\operatorname{div}, \Omega, \mathbb{M}): \sigma_{i j} n_{j}=g_{i} \text { on } \Gamma_{2}, u \in L^{2}(\Omega, \mathbb{V}), p \in L^{2}(\Omega, \mathbb{K}) \\
\int_{\Omega} C_{i j k l} \sigma_{k l} \tau_{i j}+u_{i} \tau_{i j, j}+p_{i j} \tau_{i j}=\int_{\Gamma_{1}} u_{i}^{0} \tau_{i j} n_{j} \quad \forall \tau \in H(\operatorname{div}, \Omega, \mathbb{M}): \tau_{i j} n_{j}=0 \text { on } \Gamma_{2} \\
-\int_{\Omega} \sigma_{i j, j} v_{i}-\omega^{2} \int_{\Omega} \rho u_{i} v_{i}=\int_{\Omega} f_{i} v_{i} \quad \forall v \in L^{2}(\Omega, \mathbb{V}) \\
\int_{\Omega} \sigma_{i j} q_{i j}=0 \quad \forall q \in L^{2}(\Omega, \mathbb{K})
\end{array}\right.
$$

In the above, $H(\operatorname{div}, \Omega, \mathbb{M})$ denotes the space of tensor-valued square integrable fields with a square integrable divergence. The formulation for the static case can again be derived formally by looking for a stationary point of the so-called Generalized Hellinger-Reissner functional, and it is frequently identified as the Generalized Hellinger-Reissner Variational Principle.

In the analysis presented in this paper, we consider the static case only and, for the sake of simplicity we assume that the body is fixed on the entire boundary $\partial \Omega$, i.e. $\Gamma_{2}=\emptyset$.

To connect with the notations used in $[6],[2]$, we denote by $A$ the compliance operator.

$$
A: \mathbb{M} \ni \varepsilon_{i j} \longrightarrow \sigma_{i j}=C_{i j k l} \varepsilon_{k l} \in \mathbb{M}
$$

The operator $A$ maps tensors to tensors, is bounded, symmetric and, for piecewise constant material properties, uniformly positive definite. Symmetry properties (1.3) imply that $A$ maps $\mathbb{S}$ into itself. In the isotropic case, $A$ has the form

$$
A \sigma=\frac{1}{2 \mu}\left(\sigma-\frac{\lambda}{2 \mu+n \lambda} \operatorname{Tr}(\sigma) I\right)
$$

where $\lambda(x), \mu(x)$ are the Lamé coefficients.

We can rewrite then formulation (1.5) with a more compact, index free notation.

Find $\sigma \in H(\operatorname{div}, \Omega ; \mathbb{S})$, and $u \in L^{2}(\Omega ; \mathbb{V})$, satisfying

$$
\begin{gathered}
\int_{\Omega}(A \sigma: \tau+\operatorname{div} \tau \cdot u) d x=0, \quad \tau \in H(\operatorname{div}, \Omega ; \mathbb{S}) \\
\int_{\Omega} \operatorname{div} \sigma \cdot v d x=\int_{\Omega} f \cdot v d x, \quad v \in L^{2}(\Omega ; \mathbb{V})
\end{gathered}
$$


Similarly, the formulation (1.6) becomes to seek $\sigma \in H(\operatorname{div}, \Omega ; \mathbb{M}), u \in L^{2}(\Omega ; \mathbb{V})$, and $p \in L^{2}(\Omega ; \mathbb{K})$ satisfying

$$
\begin{gathered}
\int_{\Omega}(A \sigma: \tau+\operatorname{div} \tau \cdot u+\tau: p) d x=0, \quad \tau \in H(\operatorname{div}, \Omega ; \mathbb{M}), \\
\int_{\Omega} \operatorname{div} \sigma \cdot v d x=\int_{\Omega} f \cdot v d x, \quad v \in L^{2}(\Omega ; \mathbb{V}), \\
\int_{\Omega} \sigma: q d x=0, \quad q \in L^{2}(\Omega ; \mathbb{K}) .
\end{gathered}
$$

Dual-Mixed Formulation with Weakly Imposed Symmetry for Plane Elasticity. In two space dimensions, the skew-symmetric tensors involve a single non-zero component $p$,

$$
\left(\begin{array}{cc}
0 & p \\
-p & 0
\end{array}\right)
$$

The formulation (1.8) reduces to seek $\sigma \in H(\operatorname{div}, \Omega ; \mathbb{M}), u \in L^{2}(\Omega ; \mathbb{V})$, and $p \in$ $L^{2}(\Omega)$ satisfying

$$
\begin{gathered}
\int_{\Omega}\left(A \sigma: \tau+\operatorname{div} \tau \cdot u-S_{1} \tau p\right) d x=0, \quad \tau \in H(\operatorname{div}, \Omega ; \mathbb{M}), \\
\int_{\Omega} \operatorname{div} \sigma \cdot v d x=\int_{\Omega} f \cdot v d x, \quad v \in L^{2}(\Omega ; \mathbb{V}), \\
\int_{\Omega} S_{1} \sigma q d x=0, \quad q \in L^{2}(\Omega) .
\end{gathered}
$$

where operator $S_{1}$ maps a real $2 \times 2$ matrix to a real number. For any $\sigma \in \mathbb{R}^{2 \times 2}$,

$$
S_{1} \sigma=\sigma_{12}-\sigma_{21} .
$$

1.3. Review of the existing work. Besides the Hellinger-Reissner and generalized Hellinger-Reissner principles discussed in the previous sections, there are other variational formulations for elasticity, see [18].

Stable finite element discretizations based on (1.7) are difficult to construct. A detailed description of related challenges can be founded in [14]. A stable discretization based on the strong enforcement of symmetry condition was developed in [2]. For the lowest order element, the number of degrees of freedom for stress tensor in $3 \mathrm{D}$ is 162 .

Meanwhile, a number of authors have developed approximation schemes based on (1.8). See $[5,6,14,1,3,4,15,11,17,20,21,22,23]$. For a brief description of these methods, we refer to the introduction in [6]. Recently, Cockburn, Gopalakrishnan and Guzman have developed a new mixed method for linear elasticity using a hybridized formulation based on (1.8) in [10].

The work presented in this paper is based on mixed finite element methods developed by Arnold, Falk and Winther for the formulation $(1.8)$ in $[5,14,6]$. We restrict our analysis to plane linear elasticity problem only.

At the first glance, a generalization to elements of variable order seems to be straightforward. And this is indeed the case from the implementation point of view. The stability analysis, however, is much more difficult. The canonical projection operators defined in [5] do not commute with exterior derivative operators for variable order elements, a property essential in the proof of discrete stability. In [19], we resolved the problem by invoking projection based (PB) interpolation 
operators. Unfortunately, the PB operators do not commute with the operator $S_{n-2}$, invalidating construction of operator $\tilde{W}_{h}$ introduced in the stability analysis presented in [5] We resolved the problem in [19] by designing a new operator $\tilde{W}_{h}$. However, in our first contribution we were only able to prove the well-definedness of $\tilde{W}_{h}$ for $n=2$ with orders varying from 0 to 3 . In this paper, we propose PB operators of new type and a new operator $\tilde{W}_{h}$ that enable the $h$-stability analysis for $2 \mathrm{D}$ elements of variable order ${ }^{1}$. Additionally, we analyze curvilinear elements and establish conditions for their asymptotic $h$-stability.

1.4. Scope of the paper. An outline of the paper is as follows. Section 2 introduces notations. In Section 3, we discuss curvilinear meshes and geometry assumptions. Section 4 reviews definitions of finite element spaces on a reference triangle, on a (physical) curved triangle, and on curved meshes. In Section 5, we return to the mixed formulation for plane elasticity with weakly imposed symmetry, and recall Brezzi's conditions for stability. In Section 6, we establish necessary results for proving the stability. We design new PB operators and a new operator $\tilde{W}_{h}$. In Section 7 we prove that the Brezzi conditions are satisfied asymptotically, i.e. for meshes that are sufficiently fine. We conclude the paper with numerical experiments that illustrate and verify the presented theoretical results.

\section{Notations}

For $U$, a bounded open subset in $\mathbb{R}^{n}$, we define:

$$
C^{k}(\bar{U})=\left\{u \in C^{k}(U): D^{\alpha} \text { is uniformly continuous on } U, \forall|\alpha| \leq k\right\} .
$$

We also define

$$
C^{k, 1}(\bar{U})=\left\{u \in C^{k}(\bar{U}): D^{\alpha} \text { is Lipschitz on } U, \forall|\alpha|=k\right\} .
$$

$\mathbb{M}$ will denote the space of $2 \times 2$ real matrices. For any vector space $\mathbf{X}$, we denote by $L^{2}(\Omega ; \mathbf{X})$ the space of square-integrable vector fields on $\Omega$ with values in $\mathbf{X}$. In the paper, $\mathbf{X}$ will be $\mathbb{R}, \mathbb{R}^{2}$, or $\mathbb{M}$. When $\mathbf{X}=\mathbb{R}$, we will write $L^{2}(\Omega)$. The corresponding norms will be denoted with the same symbol $L^{2}(\Omega ; \mathbb{M})$. The corresponding Sobolev space of order $m$, denoted $H^{m}(\Omega ; \mathbf{X})$, is a subspace of $L^{2}(\Omega ; \mathbf{X})$ consisting of functions with all partial derivatives of order less than or equal to $m$ in $L^{2}(\Omega ; \mathbf{X})$. The corresponding norm will be denoted by $\|\cdot\|_{H^{m}(\Omega)}$. The space $H(\operatorname{div}, \Omega ; \mathbb{M})$ is defined by

$$
H(\operatorname{div}, \Omega ; \mathbb{M})=\left\{\sigma \in L^{2}(\Omega ; \mathbb{M}): \operatorname{div} \sigma \in L^{2}\left(\Omega ; \mathbb{R}^{2}\right)\right\}
$$

where divergence of a matrix field is the vector field obtained by applying operator div row-wise, i.e.,

$$
\operatorname{div} \sigma=\left(\frac{\partial \sigma_{11}}{\partial x_{1}}+\frac{\partial \sigma_{12}}{\partial x_{2}}, \quad \frac{\partial \sigma_{21}}{\partial x_{1}}+\frac{\partial \sigma_{22}}{\partial x_{2}}\right)^{\top} .
$$

We introduce also a special space

$$
H(\Omega)=\left\{\omega \in H(\operatorname{div}, \Omega):\left.\omega\right|_{\partial \Omega} \in L^{2}\left(\partial \Omega ; \mathbb{R}^{2}\right)\right\}
$$

with the norm,

$$
\|\omega\|_{H(\Omega)}=\|\omega\|_{H(\operatorname{div}, \Omega)}+\|\omega\|_{L^{2}(\partial \Omega)}, \forall \omega \in H(\Omega) .
$$

\footnotetext{
${ }^{1}$ With an upper bound on the polynomial order.
} 
For any scalar function $u$, we denote

$$
\operatorname{curl} u=\left(\frac{\partial u}{\partial x_{2}},-\frac{\partial u}{\partial x_{1}}\right)^{\top} .
$$

For any vector valued function $\omega$ with values in $\mathbb{R}^{2}$, we denote

$$
\operatorname{curl} \omega=\left[\begin{array}{lr}
\frac{\partial \omega_{1}}{\partial x_{2}}, & -\frac{\partial \omega_{1}}{\partial x_{1}} \\
\frac{\partial \omega_{2}}{\partial x_{2}}, & -\frac{\partial \omega_{2}}{\partial x_{1}}
\end{array}\right] .
$$

Finally, by $\|\cdot\|$ we denote the standard 2-norm for vectors and matrices.

\section{Curvilinear Meshes}

Let $\hat{T} \subset \mathbb{R}^{2}$ be the master triangle,

$$
\hat{T}:=\left\{\left(\hat{x}_{1}, \hat{x}_{2}\right): \hat{x}_{1}, \hat{x}_{2}, 1-\hat{x}_{1}-\hat{x}_{2} \geq 0\right\}
$$

We denote the set of subsimplexes of dimension $k$ of $\hat{T}$ by $\triangle_{k}(\hat{T}), k=0,1,2$, and the set of all subsimplexes of $\hat{T}$ by $\triangle(\hat{T}) . \triangle_{0}(\hat{T})$ consists of all vertices of $\hat{T}, \triangle_{1}(\hat{T})$ consists of all edges of $\hat{T}$, and $\triangle_{2}(\hat{T})=\{\hat{T}\}$.

\subsection{Mesh regularity assumptions.}

Definition 3.1. (curved triangle) A closed set $T \subset \mathbb{R}^{2}$ is a curved triangle if there exists a $C^{1}$-diffeomorphism $G_{T}$ from reference triangle $\hat{T}$ onto $T$. This means that $G_{T}$ is a bijection from $\hat{T}$ to $T$ such that $G_{T} \in C^{1}(\hat{T})$ and $G_{T}^{-1} \in C^{1}(T)$. We assume additionally that $\operatorname{det}\left(D G_{T}(\hat{\mathbf{x}})\right)>0$ for any $\hat{\mathbf{x}} \in \hat{T}$.

We represent $G_{T}$ in the form

$$
G_{T}=\tilde{G}_{T}+\Phi_{T}
$$

where $\tilde{G}_{T}: \hat{\boldsymbol{x}} \rightarrow B_{T} \hat{\boldsymbol{x}}+b_{T}, B_{T}=D G_{T}(\hat{\boldsymbol{p}})$ with $\hat{\boldsymbol{p}}$ being the centroid of $\hat{T}$, and $\Phi_{T}$ a $C^{1}$-mapping from $\hat{T}$ into $\mathbb{R}^{2}$. The images of edges and vertices of $\hat{T}$ by $G_{T}$ are edges and vertices of $T$ respectively. We denote $\triangle_{i}(T)=G_{T}\left(\triangle_{i}(\hat{T})\right), i=0,1,2$, and $\triangle(T)=G_{T}(\triangle(\hat{T}))$.

Definition 3.1 is practically identical with the definition of curved finite elements introduced in [7].

Definition 3.2. A curved triangle $T$ is of class $C^{k}, k \geq 1$, if the mapping $G_{T} \in$ $C^{k}(\hat{T})$. Similarly, a curved triangle $T$ is of class $C^{k, 1}, k \geq 1$, if the mapping $G_{T} \in$ $C^{k, 1}(\hat{T})$.

We define $\mathcal{T}_{h}$ to be a finite set of curved triangles $T$, where $h$ denotes the maximal distance between two vertices of $T \in \mathcal{T}_{h}$. We define vertices of $\mathcal{T}_{h}$ to be vertices of $T \in \mathcal{T}_{h}$, and we define curves of $\mathcal{T}_{h}$ to be edges of $T \in \mathcal{T}_{h}$. We assume that any edge of $T \in \mathcal{T}_{h}$ is either an edge of another curved triangle in $\mathcal{T}_{h}$, or part of the boundary of $\mathcal{T}_{h}$.

Each curve of $\mathcal{T}_{h}$ is parametrized with a map from the reference unit interval into $\mathbb{R}^{2}$,

$$
[0,1] \ni s \rightarrow \boldsymbol{x}_{e}(s) \in \mathbb{R}^{2}
$$

The parametrization determines the orientation of the curve. 
Let $\zeta(s)$ be the local parameterization for a particular edge of a curved triangle $T \in \mathcal{T}_{h}$, occupied by a curve $e$ of $\mathcal{T}_{h}$. This means that $\zeta(s)$ is an affine mapping from the reference interval onto an edge of the reference triangle $\hat{T}$, whose image under the mapping $G_{T}$ is exactly the particular edge of $T$. We can choose $\zeta(s)$ so that $G_{T}(\zeta(s))$ has the same orientation as $\mathbf{x}_{e}(s)$.

Definition 3.3. ( $C^{0}$-compatible mesh) $\mathcal{T}_{h}$ is called a $C^{0}$-compatible mesh if, for any curve $e$ and any curved triangle $T$ which contains $e$ as an edge, there is a local parameterization $\zeta(s)$ of $e$ satisfying

$$
G_{T}(\zeta(s))=\mathbf{x}_{e}(s)
$$

The concept is illustrated in Fig 1.

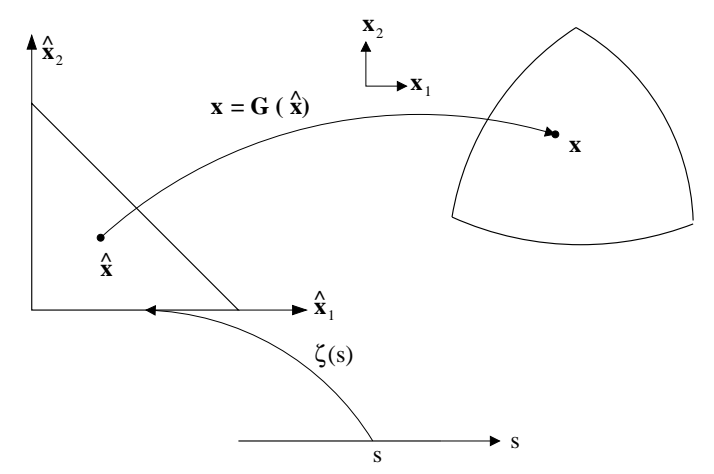

Figure 1. Compatibility of edge and triangle parametrizations.

We denote

$$
c_{h}:=\sup _{T \in \mathcal{T}_{h}}\left(\left(\sup _{\hat{\mathbf{x}} \in \hat{T}}\left\|D \Phi_{T}(\hat{\mathbf{x}})\right\|\right)\left\|B_{T}^{-1}\right\|\right)
$$

For each $T \in \mathcal{T}_{h}$, we define $\tilde{T}=\tilde{G}_{T}(\hat{T})$. We denote by $\tilde{h}_{T}$ the diameter of $\tilde{T}$ and by $\tilde{\rho}_{T}$ the diameter of the sphere inscribed in $\tilde{T}$.

We define $\triangle_{i}\left(\mathcal{T}_{h}\right)=\bigcup_{T \in \mathcal{T}_{h}} \triangle_{i} T$, and $\triangle\left(\mathcal{T}_{h}\right)=\bigcup_{T \in \mathcal{T}_{h}} \triangle T$.

Remark 3.4. In order to simplify analysis, compared with [7], our definition of (3.2) replaces $\left\|D \Phi_{T} \cdot B_{T}^{-1}\right\|$ with the upper bound $\left\|D \Phi_{T}\right\| \cdot\left\|B_{T}^{-1}\right\|$.

Definition 3.5. The family $\left(\mathcal{T}_{h}\right)_{h}$ of $C^{0}$-compatible meshes is said to be regular if

$$
\sup _{h} \sup _{T \in \mathcal{T}_{h}} \tilde{h}_{T} / \tilde{\rho}_{T}=\sigma<\infty, \text { and } \lim _{h \rightarrow 0} c_{h}=0
$$

Lemma 3.6. There exist $c_{1}, c_{2}>0$ such that, for any triangle $T$,

$$
c_{1}\left\|B_{T}\right\| \cdot\left\|B_{T}^{-1}\right\| \leq \tilde{h}_{T} / \tilde{\rho}_{T} \leq c_{2}\left\|B_{T}\right\| \cdot\left\|B_{T}^{-1}\right\|,
$$

where $\mathbf{x}=B_{T} \hat{\mathbf{x}}+b_{T}$ is the affine homeomorphism from $\hat{T}$ to $T$.

Proof. $\tilde{h}_{T} / \tilde{\rho}_{T} \leq c_{2}\left\|B_{T}\right\| \cdot\left\|B_{T}^{-1}\right\|$ comes from the geometric meaning of singular values of matrix $B_{T} \cdot c_{1}\left\|B_{T}\right\| \cdot\left\|B_{T}^{-1}\right\| \leq \tilde{h}_{T} / \tilde{\rho}_{T}$ is a consequence of Theorem 3.1.3 in $[9]$. 
Lemma 3.7. Let family $\left(\mathcal{T}_{h}\right)_{h}$ be regular. Then, for any indices $i, j, k, l \in\{1,2,3\}$, we have

$$
\lim _{h \rightarrow 0} \sup _{T \in \mathcal{T}_{h}} \sup _{\hat{\mathbf{x}} \in \hat{T}}\left|\frac{\left(B_{T}\right)_{i j}\left(D \Phi_{T}(\hat{\mathbf{x}})\right)_{k l}}{\operatorname{det}\left(B_{T}\right)}\right|=0
$$

Proof. For any $T \in \mathcal{T}_{h}$ and any $\hat{\mathbf{x}} \in \hat{T}$, we have

$$
\left|\frac{\left(B_{T}\right)_{i j}\left(D \Phi_{T}(\hat{\mathbf{x}})\right)_{k l}}{\operatorname{det}\left(B_{T}\right)}\right|=\left|\left(D \Phi_{T}(\hat{\mathbf{x}})\right)_{k l}\right||| B_{T}^{-1} \| \cdot\left|\left(B_{T}\right)_{i j} / \operatorname{det}\left(B_{T}\right)\right| \frac{1}{\left\|B_{T}^{-1}\right\|} .
$$

Since $\left\|B_{T}^{-1}\right\| \cdot\left\|B_{T}\right\| \geq 1, \frac{1}{\left\|B_{T}^{-1}\right\|} \leq\left\|B_{T}\right\|$. Consequently,

$$
\left|\frac{\left(B_{T}\right)_{i j}\left(D \Phi_{T}(\hat{\mathbf{x}})\right)_{k l}}{\operatorname{det}\left(B_{T}\right)}\right| \leq\left(\left\|D \Phi_{T}(\hat{\mathbf{x}})\right\| \cdot\left\|B_{T}^{-1}\right\|\right)\left\|B_{T}\right\|^{2} /\left|\operatorname{det}\left(B_{T}\right)\right| .
$$

Since $\sup _{h} \sup _{T \in \mathcal{T}_{h}} \tilde{h}_{T} / \tilde{\rho}_{T}=\sigma<\infty,\left\|B_{T}\right\|^{2} /\left|\operatorname{det}\left(B_{T}\right)\right| \leq \frac{c}{\sigma^{2}}$ for some constant $c>0$.

Since $\lim _{h \rightarrow 0} c_{h}=0$, we have $\lim _{h \rightarrow 0} \sup _{T \in \mathcal{T}_{h}} \sup _{\hat{\mathbf{x}} \in \hat{T}}\left|\frac{\left(B_{T}\right)_{i j}\left(D \Phi_{T}(\hat{\mathbf{x}})\right)_{k l}}{\operatorname{det}\left(B_{T}\right)}\right|=0$.

Lemma 3.8. If a family $\left(\mathcal{T}_{h}\right)_{h}$ is regular, then we have

$$
\lim _{h \rightarrow 0} \sup _{T \in \mathcal{T}_{h}} \sup _{\hat{\mathbf{x}} \in \hat{T}}\left\|B_{T}\left(D G_{T}(\hat{\mathbf{x}})\right)^{-1}-I\right\|=\lim _{h \rightarrow 0} \sup _{T \in \mathcal{T}_{h}} \sup _{\hat{\mathbf{x}} \in \hat{T}}\left\|\left(D G_{T}(\hat{\mathbf{x}})\right)^{-1} B_{T}-I\right\|=0 .
$$

Proof. For any $T \in \mathcal{T}_{h}$ and any $\hat{\mathbf{x}} \in \hat{T}$, we have

$\left\|B_{T}\left(D G_{T}(\hat{\mathbf{x}})\right)^{-1}-I\right\|=\left\|B_{T}\left(B_{T}+D \Phi_{T}(\hat{\mathbf{x}})\right)^{-1}-I\right\|=\left\|\left(I+D \Phi_{T}(\hat{\mathbf{x}}) B_{T}^{-1}\right)^{-1}-I\right\|$.

Since $c_{h} \rightarrow 0$ as $h \rightarrow 0$, we have

$$
\lim _{h \rightarrow 0} \sup _{T \in \mathcal{T}_{h}} \sup _{\hat{\mathbf{x}} \in \hat{T}}\left\|B_{T}\left(D G_{T}(\hat{\mathbf{x}})\right)^{-1}-I\right\|=0 .
$$

Proof of the second property is fully analogous.

Lemma 3.9. If a family $\left(\mathcal{T}_{h}\right)_{h}$ is regular, then we have

$\lim _{h \rightarrow 0} \sup _{T \in \mathcal{T}_{h}} \sup _{\hat{\mathbf{x}} \in \hat{T}}\left|\operatorname{det}\left(D G_{T}(\hat{\mathbf{x}}) B_{T}^{-1}\right)-1\right|=\lim _{h \rightarrow 0} \sup _{T \in \mathcal{T}_{h}} \sup _{\hat{\mathbf{x}} \in \hat{T}}\left|\operatorname{det}\left(B_{T}\left(D G_{T}(\hat{\mathbf{x}})\right)^{-1}\right)-1\right|=0$.

Proof. Since $\lim _{h \rightarrow 0} c_{h}=0$, we immediately have

$$
\lim _{h \rightarrow 0} \sup _{T \in \mathcal{T}_{h}} \sup _{\hat{\mathbf{x}} \in \hat{T}}\left|\operatorname{det}\left(D G_{T}(\hat{\mathbf{x}}) B_{T}^{-1}\right)-1\right|=0 .
$$

According to Lemma 3.8, we have

$$
\lim _{h \rightarrow 0} \sup _{T \in \mathcal{T}_{h}} \sup _{\hat{\mathbf{x}} \in \hat{T}}\left|\operatorname{det}\left(B_{T}\left(D G_{T}(\hat{\mathbf{x}})\right)^{-1}\right)-1\right|=0 .
$$


3.2. Mesh generation. From now on, we assume that the domain $\Omega$ is a (curvilinear) polygon, and that it can be meshed with a regular family $\left(\mathcal{T}_{h}\right)_{h}$ of $C^{0}$ compatible meshes (i.e. $\bar{\Omega}=\bigcup_{T \in \mathcal{T}_{h}} T$, for all $h$ ) that satisfy the regularity assumptions discussed in the previous section.

We will outline now shortly how one can generate such meshes in practice. Suppose the domain $\Omega$ has been meshed with a $C^{0}$-compatible initial mesh $\mathcal{T}_{\text {int }}$, $\bar{\Omega}=\bigcup_{i=1}^{m} T_{i}$, where $\left\{T_{i}\right\}_{i=1}^{m}$ are curved triangles of class $C^{1,1}$. We denote by $\left\{G_{1}, \cdots, G_{m}\right\}$ the mappings from $\hat{T}$ to $\left\{T_{1}, \cdots, T_{m}\right\}$. Then $G_{i} \in C^{1,1}(\hat{T})$ and $G_{i}^{-1} \in C^{1}\left(T_{i}\right)$ for any $1 \leq i \leq m$. For examples of techniques to generate an initial mesh satisfying the assumptions above, see [12].

Lemma 3.10. Let $T=G_{T}(\hat{T})$ be a closed triangle in $\mathbb{R}^{2}$ with $G_{T} \in C^{1,1}(\hat{T})$. Let $\check{\sigma}>0$ be a positive constant. For any $\breve{h}>0$, we denote by $\check{T}_{\breve{h}}$ any triangle contained in $\hat{T}$ such that the diameter of $\check{T}_{\breve{h}}$ is $\breve{h}$, and $\breve{h} / \check{\rho} \leq \check{\sigma}$ where $\check{\rho}$ is the diameter of the sphere inscribed in $\check{T}_{\breve{h}}$. Let $\hat{T} \ni \hat{\mathbf{x}} \rightarrow H \hat{\mathbf{x}}=\check{B} \hat{\mathbf{x}}+\check{b}$ be an affine mapping from $\hat{T}$ onto $\check{T}_{\breve{h}}$. Let $\hat{\mathbf{p}}$ be the centroid of $\hat{T}$. We put $B=D\left(G_{T} \circ H\right)(\hat{\mathbf{p}}), b=\left(G_{T} \circ H\right)(\hat{\mathbf{p}})$, and $\Psi(\hat{\mathbf{x}})=\left(G_{T} \circ H\right)(\hat{\mathbf{x}})-\check{B}(\hat{\mathbf{x}}-\hat{\mathbf{p}})-b$.

Then, we have

$$
\left(\sup _{\hat{\mathbf{x}} \in \hat{T}}\|D \Psi(\hat{\mathbf{x}})\|\right)\left\|B^{-1}\right\| \leq C \check{h},
$$

where $C$ is a constant independent of $\check{h}$, and $\check{T}_{\breve{h}}$.

Proof. Obviously, $B=D G_{T}(H(\hat{\mathbf{p}})) \check{B}$. So $B^{-1}=\check{B}^{-1}\left(D G_{T}(H(\hat{\mathbf{p}}))\right)^{-1}$. We have

$$
D \Psi(\hat{\mathbf{x}})=\left(D G_{T}(H(\hat{\mathbf{x}}))-D G_{T}(H(\hat{\mathbf{p}}))\right) \check{B}
$$

Since $G_{T}$ is a $C^{1}$-diffeomorphism from $\hat{T}$ onto $T$, we have $\sup _{\hat{\mathbf{x}} \in \hat{T}}\left\|\left(D G_{T}(\hat{\mathbf{x}})\right)^{-1}\right\|<$ $\infty$. With $G_{T} \in C^{1,1}(\hat{T})$, we also have

$$
\left\|D G_{T}(H(\hat{\mathbf{x}}))-D G_{T}(H(\hat{\mathbf{p}}))\right\| \leq M\|H(\hat{\mathbf{x}})-H(\hat{\mathbf{p}})\| \quad \text { for any } \hat{\mathbf{x}} \in \hat{T}
$$

where $M$ is the Lipschitz constant for all first order derivatives of $G_{T}$ on $\hat{T}$.

With $\check{h} / \check{\rho} \leq \check{\sigma}$, we obtain that $\|\check{B}\| \cdot\left\|\check{B}^{-1}\right\| \leq \frac{c}{\check{\sigma}}$, for some $c>0$. With $\check{h}$ denoting the diameter of $\check{T}_{\breve{h}}$, we have $\|H(\hat{\mathbf{x}})-H(\hat{\mathbf{p}})\| \leq \check{h}$ for any $\hat{\mathbf{x}} \in \check{T}_{\breve{h}}$. The definition of $\Psi$ implies then

$$
\left(\sup _{\hat{\mathbf{x}} \in \hat{T}}\|D \Psi(\hat{\mathbf{x}})\|\right)\left\|B^{-1}\right\| \leq \frac{c}{\check{\sigma}} M\left(\sup _{\hat{\mathbf{y}} \in \hat{T}}\left\|\left(D G_{T}(\hat{\mathbf{y}})\right)^{-1}\right\|\right) \check{h} .
$$

Setting $C=\frac{c}{\check{\sigma}} M\left(\sup _{\hat{\mathbf{y}} \in \hat{T}}\left\|\left(D G_{T}(\hat{\mathbf{y}})\right)^{-1}\right\|\right)$ finishes the proof.

Let $\hat{T}_{i}$ be now multiple copies of the reference triangle corresponding to the initial mesh, $T_{i}=G_{i}\left(\hat{T}_{i}\right), i=1, \ldots, m$.

Definition 3.11. (Regular triangular meshes in the reference space) A family of triangulations $\left(\hat{\mathcal{T}}_{i, \breve{h}}\right)_{\breve{h}}$ of reference triangles $\hat{T}_{i}$ is said to be regular provided two conditions are satisfied:

(i) Partitions of edges of $\hat{T}_{i}$ mapped into the same edge in the physical space are identical.

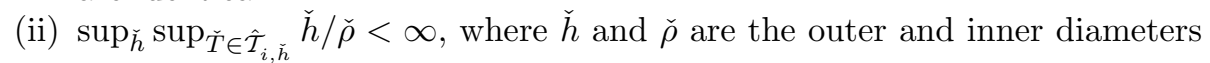
of $\check{T}$. 
Obviously, uniform refinements of reference triangles ${ }^{2}$ are regular. A number of adaptive refinement algorithms produces regular meshes as well. To this class belong e.g. Rivara's algorithm (bisection by the longest edge), Arnold's algorithm (bisection by the newest edge), the Delaunay triangulation (see [8]).

Using Lemma 3.10 and the fact that $G_{i}$ is $C^{1}$-diffeomorphism from $\hat{T}$ to $T_{i}, 1 \leq$ $i \leq m$, we easily conclude that any regular refinements in the reference space produce curvilinear meshes that satisfy our mesh regularity assumptions.

\subsection{Properties of Sobolev spaces on curved and reference triangles.}

Lemma 3.12. Let $T$ be a curved triangle. For any $\omega \in H^{1}\left(T ; \mathbb{R}^{2}\right)$, we define $\hat{\omega}(\hat{\mathbf{x}})$ on $\hat{T}$ by

$$
\omega(\mathbf{x})=\frac{D G_{T}(\hat{\mathbf{x}})}{\operatorname{det}\left(D G_{T}(\hat{\mathbf{x}})\right)} \hat{\omega}(\hat{\mathbf{x}}) \quad \hat{\mathbf{x}} \in \hat{T} .
$$

Then $\hat{\omega}(\hat{\mathbf{x}}) \in H(\hat{T})$. Divergence transforms by the classical Piola's rule:

$$
\operatorname{div\omega }(\mathbf{x})=\left(\operatorname{det}\left(D G_{T}(\hat{\mathbf{x}})\right)\right)^{-1} \operatorname{div}_{\hat{\mathbf{x}}} \hat{\omega}(\hat{\mathbf{x}})
$$

for $\hat{\mathbf{x}} \in \hat{T}$ almost everywhere.

Proof. Notice that $\hat{\omega}(\hat{\mathbf{x}})=\operatorname{det}\left(D G_{T}(\hat{\mathbf{x}})\right)\left(D G_{T}(\hat{\mathbf{x}})\right)^{-1} \omega(\mathbf{x})$ for any $\hat{\mathbf{x}} \in \hat{T}$. It is straightforward to see that $\operatorname{det}\left(D G_{T}(\hat{\mathbf{x}})\right)\left(D G_{T}(\hat{\mathbf{x}})\right)^{-1}$ is a matrix whose entries contain first order partial derivatives of $G_{T}(\hat{x})$. Notice that

$$
\hat{\omega}(\hat{\mathbf{x}})=\operatorname{det}\left(D G_{T}(\hat{\mathbf{x}})\right)\left(D G_{T}(\hat{\mathbf{x}})\right)^{-1} \omega(\mathbf{x})
$$

is the standard pull back mapping from $H(\operatorname{div}, T)$ to $H\left(\operatorname{div}_{\hat{\mathbf{x}}}, \hat{T}\right)$. So we immediately have $\operatorname{div} \omega(\mathbf{x})=\frac{1}{\operatorname{det}\left(D G_{T}(\hat{\mathbf{x}})\right)} \operatorname{div}_{\hat{\mathbf{x}}} \hat{\omega}(\hat{\mathbf{x}})$ for $\hat{\mathbf{x}} \in \hat{T}$ almost everywhere. Since $G_{T}$ is a $C^{1}$-diffeomorphism from $\hat{T}$ to $T$, we can conclude that $\hat{\omega}(\hat{\mathbf{x}}) \in H(\operatorname{div}, \hat{T})$.

Let $e \in \triangle_{1}(T)$. We denote by $\zeta(s):[0,1] \rightarrow \hat{e}$, the local affine parameterization of $\hat{e}$. We have then

$$
\begin{aligned}
\|\hat{\omega}\|_{L^{2}(\hat{e})}^{2}= & \int_{[0,1]}(\hat{\omega}(\zeta(s)))^{\top} \hat{\omega}(\zeta(s))\|\dot{\zeta}(s)\| d s \\
= & \int_{[0,1]}\left(\operatorname{det}\left(D G_{T}(\zeta(s))\right)\right)^{2} \omega\left(G_{T}(\zeta(s))\right)^{\top}\left[D G_{T}(\zeta(s))^{-\top} D G_{T}(\zeta(s))^{-1}\right] \\
& \omega\left(G_{T}(\zeta(s))\right)\left\|\left(D G_{T}(\zeta(s))\right)^{-1}\left[D G_{T}(\zeta(s)) \dot{\zeta}(s)\right]\right\| d s .
\end{aligned}
$$

Since $G_{T}$ is a $C^{1}$-diffeomorphism from $\hat{T}$ to $T$, we can conclude that $\left.\hat{\omega}\right|_{\partial \hat{T}} \in$ $L^{2}\left(\partial \hat{T} ; \mathbb{R}^{2}\right)$. So $\hat{\omega} \in H(\hat{T})$.

Lemma 3.13. There exist $\delta>0$ and $C>0$ such that, for any $h<\delta$ and $T \in \mathcal{T}_{h}$,

$$
\|\omega\|_{L^{2}(T)} \leq C\|\hat{\omega}\|_{L^{2}(\hat{T})}, \forall \omega \in L^{2}\left(T ; \mathbb{R}^{2}\right)
$$

where $\frac{D G_{T}(\hat{\mathbf{x}}) \hat{\omega}(\hat{\mathbf{x}})}{\operatorname{det}\left(D G_{T}(\hat{\mathbf{x}})\right)}=\omega(\mathbf{x})$ for any $\hat{\mathbf{x}} \in \hat{T}$. If $T$ is a triangle for any $T \in \mathcal{T}_{h}$ and $h>0$, then the above inequality holds for any $h>0$.

\footnotetext{
${ }^{2}$ With the same number of divisions for each reference triangle.
} 
Proof.

$$
\begin{aligned}
\|\omega\|_{L^{2}(T)} & =\int_{T} \frac{1}{\operatorname{det}\left(D G_{T}(\hat{\mathbf{x}})\right)^{2}}\left[D G_{T}(\hat{\mathbf{x}}) \hat{\omega}(\hat{\mathbf{x}})\right]^{\top} D G_{T}(\hat{\mathbf{x}}) \hat{\omega}(\hat{\mathbf{x}}) d \mathbf{x} \\
& =\int_{\hat{T}} \operatorname{det}\left(\left(D G_{T}\right)^{-1}\right) \hat{\omega}^{\top}\left[D G_{T}^{\top} D G_{T}\right] \hat{\omega} d \hat{\mathbf{x}} \\
& =\int_{\hat{T}} \operatorname{det}\left(D G_{T}^{-1} B_{T}\right) \operatorname{det}\left(B_{T}^{-1}\right) \hat{\omega}^{\top} B_{T}^{\top}\left[B_{T}^{-\top} D G_{T}^{\top} D G_{T} B_{T}^{-1}\right] B_{T} \hat{\omega} d \hat{\mathbf{x}} .
\end{aligned}
$$

Since $c_{h} \rightarrow 0$ as $h \rightarrow 0, \lim _{h \rightarrow 0} \sup _{T \in \mathcal{T}_{h}} \sup _{\hat{\mathbf{x}} \in \hat{T}}\left\|B_{T}^{-\top} D G_{T}^{\top} D G_{T} B_{T}^{-1}-I\right\|=0$. By Lemma 3.9, we have $\lim _{h \rightarrow 0} \sup _{T \in \mathcal{T}_{h}} \sup _{\hat{\mathbf{x}} \in \hat{T}}\left|\operatorname{det}\left(D G_{T}^{-1} B_{T}\right)-1\right|=0$.

Since $\left(\mathcal{T}_{h}\right)_{h}$ is regular, there is a constant $\sigma>0$ such that $\sigma_{1} / \sigma_{2} \leq \sigma$ for any $T \in \mathcal{T}_{h}$, where $\sigma_{1}$ and $\sigma_{2}$ denote the biggest and smallest singular value of the corresponding matrix $B_{T}$. Then $\left\|B_{T}^{\top}\right\|=\left\|B_{T}\right\|=\sigma_{1}$ and $\operatorname{det}\left(B_{T}\right)=\sigma_{1} \cdot \sigma_{2}$. So $\left\|B_{T}^{\top}\right\| \cdot\left\|B_{T}\right\| \operatorname{det}\left(B_{T}^{-1}\right)=\sigma_{1} / \sigma_{2} \leq \sigma$ for any $h>0$ and any $T \in \mathcal{T}_{h}$.

We can conclude thus that there exist $\delta>0$ and $C>0$ such that, for any $h<\delta$ and $T \in \mathcal{T}_{h}$,

$$
\|\omega\|_{L^{2}(T)} \leq C\|\hat{\omega}\|_{L^{2}(\hat{T})} \quad \forall \omega \in L^{2}\left(T ; \mathbb{R}^{2}\right)
$$

If, for all $h, T \in \mathcal{T}_{h}$ are (regular) triangles, the asymptotic argument is not necessary, and the above inequality holds for any $h>0$.

Lemma 3.14. There exist $\delta>0$ and $C>0$ such that, for any $h<\delta$ and $T \in \mathcal{T}_{h}$,

$$
\|\hat{\omega}\|_{H\left(d i v_{\hat{\mathbf{x}}}, \hat{T}\right)}^{2}+\|\hat{\omega}\|_{L^{2}(\partial \hat{T})}^{2} \leq C\|\omega\|_{H^{1}(T)}^{2} \quad \forall \omega \in H^{1}\left(T ; \mathbb{R}^{2}\right)
$$

where $\frac{D G_{T}(\hat{\mathbf{x}}) \hat{\omega}(\hat{\mathbf{x}})}{\operatorname{det}\left(D G_{T}(\hat{\mathbf{x}})\right)}=\omega(\mathbf{x})$ for any $\hat{\mathbf{x}} \in \hat{T}$. If $T$ is a triangle for any $T \in \mathcal{T}_{h}$ and $h>0$, then the above inequality holds for any $h>0$.

Proof.

$$
\begin{aligned}
&\|\hat{\omega}\|_{L^{2}(\hat{T})}^{2}= \int_{\hat{T}}\left(\operatorname{det}\left(D G_{T}(\hat{\mathbf{x}})\right)\right)^{2} \omega(\mathbf{x})^{\top}\left[D G_{T}(\hat{\mathbf{x}})^{-T} D G_{T}(\hat{\mathbf{x}})^{-1}\right] \omega(\mathbf{x}) d \hat{\mathbf{x}} \\
&= \int_{T} \operatorname{det}\left(D G_{T}(\hat{\mathbf{x}})\right) \omega(\mathbf{x})^{\top}\left[D G_{T}(\hat{\mathbf{x}})^{-T} D G_{T}(\hat{\mathbf{x}})^{-1}\right] \omega(\mathbf{x}) d \mathbf{x} \\
&= \int_{T} \operatorname{det}\left(D G_{T}(\hat{\mathbf{x}}) B_{T}^{-1}\right) \operatorname{det}\left(B_{T}\right) \omega(\mathbf{x})^{\top} B_{T}^{-\top} \\
& {\left[B_{T}^{\top} D G_{T}(\hat{\mathbf{x}})^{-T} D G_{T}(\hat{\mathbf{x}})^{-1} B_{T}\right] B_{T}^{-1} \omega(\mathbf{x}) d \mathbf{x} . }
\end{aligned}
$$

According to Lemma 3.8, $\lim _{h \rightarrow 0} \sup _{T \in \mathcal{T}_{h}} \sup _{\hat{\mathbf{x}} \in \hat{T}} \| B_{T}^{\top} D G_{T}(\hat{\mathbf{x}})^{-T} D G_{T}(\hat{\mathbf{x}})^{-1} B_{T}-$ $I \|=0$. By Lemma 3.9, we have $\lim _{h \rightarrow 0} \sup _{T \in \mathcal{T}_{h}} \sup _{\hat{\mathbf{x}} \in \hat{T}}\left|\operatorname{det}\left(D G_{T}(\hat{\mathbf{x}}) B_{T}^{-1}\right)-1\right|=0$.

Since $\left(\mathcal{T}_{h}\right)_{h}$ is regular, there exists a constant $\sigma>0$ such that $\sigma_{1} / \sigma_{2} \leq \sigma$ for any $T \in \mathcal{T}_{h}$, where $\sigma_{1}$ and $\sigma_{2}$ denote the biggest and smallest singular value of the matrix $B_{T}$. Then $\left\|B_{T}^{-\top}\right\|=\left\|B_{T}^{-1}\right\|=\sigma_{2}^{-1}$ and $\operatorname{det}\left(B_{T}\right)=\sigma_{1} \cdot \sigma_{2}$. So $\left\|B_{T}^{-\top}\right\| \cdot\left\|B_{T}^{-1}\right\| \operatorname{det}\left(B_{T}\right)=\sigma_{1} / \sigma_{2} \leq \sigma$. Consequently, there exist $\delta_{1}>0$ and $C_{1}>0$ such that, for any $h<\delta_{1}$ and $T \in \mathcal{T}_{h}$,

$$
\begin{aligned}
& \operatorname{det}\left(D G_{T}(\hat{\mathbf{x}}) B_{T}^{-1}\right) \operatorname{det}\left(B_{T}\right) \omega(\mathbf{x})^{\top} B_{T}^{-\top}\left[B_{T}^{\top} D G_{T}(\hat{\mathbf{x}})^{-T} D G_{T}(\hat{\mathbf{x}})^{-1} B_{T}\right] B_{T}^{-1} \omega(\mathbf{x}) \\
& \leq C_{1}^{2} \omega(\mathbf{x})^{\top} \omega(\mathbf{x})
\end{aligned}
$$


for all $\omega \in H^{1}\left(T ; \mathbb{R}^{2}\right), \mathbf{x} \in T$. We can conclude that, for any $h<\delta_{1}$ and $T \in \mathcal{T}_{h}$,

$$
\|\hat{\omega}\|_{L^{2}(\hat{T})} \leq C_{1}\|\omega\|_{L^{2}(T)} \quad \forall \omega \in H^{1}\left(T ; \mathbb{R}^{2}\right)
$$

According to Lemma 3.12, we have

$$
\begin{aligned}
\left\|\operatorname{div}_{\hat{\mathbf{x}} \hat{\omega}}\right\|_{L^{2}(\hat{T})}^{2} & =\int_{\hat{T}}\left(\operatorname{det}\left(D G_{T}(\hat{\mathbf{x}})\right)\right)^{2}(\operatorname{div} \omega(\mathbf{x}))^{2} d \hat{\mathbf{x}}=\int_{T} \operatorname{det}\left(D G_{T}(\hat{\mathbf{x}})\right)(\operatorname{div} \omega(\mathbf{x}))^{2} d \mathbf{x} \\
& =\int_{T} \operatorname{det}\left(D G_{T}(\hat{\mathbf{x}}) B_{T}^{-1}\right) \operatorname{det}\left(B_{T}\right)(\operatorname{div} \omega(\mathbf{x}))^{2} d \mathbf{x} .
\end{aligned}
$$

By Lemma 3.9, $\lim _{h \rightarrow 0} \sup _{T \in \mathcal{T}_{h}} \sup _{\hat{\mathbf{x}} \in \hat{T}}\left|\operatorname{det}\left(D G_{T}(\hat{\mathbf{x}}) B_{T}^{-1}\right)-1\right|=0$. Obviously, $\operatorname{det}\left(B_{T}\right) \leq \tilde{h}_{T}^{2}$. There must exist then $\delta_{2}>0$ and $C_{2}>0$ such that, for any $h<\delta_{2}$ and $T \in \mathcal{T}_{h}$,

$$
\operatorname{det}\left(D G_{T}(\hat{\mathbf{x}}) B_{T}^{-1}\right) \operatorname{det}\left(B_{T}\right)(\operatorname{div} \omega(\mathbf{x}))^{2} \leq C_{2}^{2} \tilde{h}_{T}^{2}(\operatorname{div} \omega(\mathbf{x}))^{2} \quad \forall \omega \in H^{1}\left(T ; \mathbb{R}^{2}\right), \mathbf{x} \in T .
$$

We conclude that, for any $h<\delta_{2}$ and $T \in \mathcal{T}_{h}$,

$$
\left\|\operatorname{div}_{\hat{\mathbf{x}}} \hat{\omega}\right\|_{L^{2}(\hat{T})} \leq C_{2} \tilde{h}_{T}\|\operatorname{div} \omega\|_{L^{2}(T)} \quad \forall \omega \in H^{1}\left(T ; \mathbb{R}^{2}\right) .
$$

We take now an arbitrary $e \in \triangle_{1}(T)$. We denote by $\zeta(s):[0,1] \rightarrow \hat{e}$ the local affine parameterization of $\hat{e}$. We have then

$$
\begin{aligned}
\|\hat{\omega}\|_{L^{2}(\hat{e})}^{2} & =\int_{[0,1]}(\hat{\omega}(\zeta(s)))^{\top} \hat{\omega}(\zeta(s))\|\dot{\zeta}(s)\| d s \\
& =\int_{[0,1]}\left(\operatorname{det}\left(D G_{T}(\zeta(s))\right)\right)^{2} \omega\left(G_{T}(\zeta(s))\right)^{\top}\left[D G_{T}(\zeta(s))^{-\top} D G_{T}(\zeta(s))^{-1}\right] \\
& =\int_{[0,1]}\left(\operatorname{det}\left(D G_{T}(\zeta(s))\right)\|\dot{\zeta}(s)\| d s\right. \\
& {\left.\left.\left[B_{T}^{\top} D G_{T}(\zeta(s))\right) B_{T}^{-1}\right)^{2}\left(\operatorname{det}\left(B_{T}\right)\right)^{2} \omega\left(G_{T}(\zeta(s))\right)^{\top} B_{T}^{-\top}(\zeta(s))^{-1} B_{T}\right] B_{T}^{-1} \omega\left(G_{T}(\zeta(s))\right)\|\dot{\zeta}(s)\| d s . }
\end{aligned}
$$

According to Lemma 3.8, we have that

$$
\lim _{h \rightarrow 0} \sup _{T \in \mathcal{T}_{h}} \sup _{e \in T} \sup _{s \in[0,1]}\left\|B_{T}^{\top} D G_{T}(\zeta(s))^{-\top} D G_{T}(\zeta(s))^{-1} B_{T}-I\right\|=0 .
$$

By Lemma 3.9, $\lim _{h \rightarrow 0} \sup _{T \in \mathcal{T}_{h}} \sup _{e \in T} \sup _{s \in[0,1]}\left|\left(\operatorname{det}\left(D G_{T}(\zeta(s))\right) B_{T}^{-1}\right)^{2}-1\right|=0$.

Consider again the singular values of $B_{T}, \sigma_{1} \geq \sigma_{2}$. Then $\left\|B_{T}^{-\top}\right\|=\left\|B_{T}^{-1}\right\|=$ $\sigma_{2}^{-1}$, and $\operatorname{det}\left(B_{T}\right)=\sigma_{1} \cdot \sigma_{2}$. So $\left\|B_{T}^{-\top}\right\| \cdot\left\|B_{T}^{-1}\right\|\left(\operatorname{det}\left(B_{T}\right)\right)^{2}=\left(\sigma_{1}\right)^{2} \leq \tilde{h}_{T}^{2}$. Consequently, there exist $\delta_{3}>0$ and $C_{3}>0$ such that, for any $h<\delta_{3}$ and $T \in \mathcal{T}_{h}$,

$$
\begin{gathered}
\left(\operatorname{det}\left(D G_{T}(\zeta(s))\right) B_{T}^{-1}\right)^{2}\left(\operatorname{det}\left(B_{T}\right)\right)^{2} \omega\left(G_{T}(\zeta(s))\right)^{\top} B_{T}^{-\top} \\
{\left[B_{T}^{\top} D G_{T}(\zeta(s))^{-\top} D G_{T}(\zeta(s))^{-1} B_{T}\right] B_{T}^{-1} \omega\left(G_{T}(\zeta(s))\right)} \\
\leq C_{3} \tilde{h}_{T}^{2} \omega(\mathbf{x})^{\top} \omega(\mathbf{x}) \quad \forall \omega \in H^{1}\left(T ; \mathbb{R}^{2}\right), e \in \triangle_{1}(T), s \in[0,1]
\end{gathered}
$$

and

$$
\begin{aligned}
\|\dot{\zeta}(s)\| & =\left\|B_{T}^{-1}\left[B_{T} D G_{T}(\zeta(s))^{-1}\right] \cdot\left[D G_{T}(\zeta(s)) \dot{\zeta}(s)\right]\right\| \\
& \leq C_{3} \tilde{h}_{T}^{-1}\left\|D G_{T}(\zeta(s)) \dot{\zeta}(s)\right\| \quad \forall e \in \triangle_{1}(T), s \in[0,1] .
\end{aligned}
$$


We can conclude that, for any $h<\delta_{3}$ and $T \in \mathcal{T}_{h}$,

$$
\begin{aligned}
\|\hat{\omega}\|_{L^{2}(\hat{e})}^{2} & \leq C_{3}^{2} \tilde{h}_{T} \int_{[0,1]} \omega\left(G_{T}(\zeta(s))\right)^{\top} \omega\left(G_{T}(\zeta(s))\right)\left\|D G_{T}(\zeta(s)) \dot{\zeta}(s)\right\| d s \\
& =C_{3}^{2} \tilde{h}_{T}\|\omega\|_{L^{2}(e)}^{2} .
\end{aligned}
$$

Obviously, $\sup _{h} \sup _{T \in \mathcal{T}_{h}} \tilde{h}_{T}<\infty$. Since $\omega \in H^{1}\left(T ; \mathbb{R}^{2}\right)$, we can use the Trace Theorem to conclude that there exist $\delta>0$ and $C>0$ such that, for any $h<\delta$ and $T \in \mathcal{T}_{h}$

$$
\|\hat{\omega}\|_{H\left(\operatorname{div}_{\hat{\mathbf{x}}}, \hat{T}\right)}^{2}+\|\hat{\omega}\|_{L^{2}(\partial \hat{T})}^{2} \leq C\|\omega\|_{H^{1}(T)}^{2} \quad \forall \omega \in H^{1}\left(T ; \mathbb{R}^{2}\right) .
$$

It is easy to see that, if $T$ is a (regular) triangle for any $T \in \mathcal{T}_{h}$ and $h>0$, then the inequality above holds for any $h>0$.

Lemma 3.15. There exist $\delta>0$ and $C>0$ such that, for any $h<\delta$ and $T \in \mathcal{T}_{h}$,

$$
\|\operatorname{curl} \omega\|_{L^{2}(T)}^{2} \leq C \tilde{h}_{T}^{-2}\left\|\operatorname{curl}_{\hat{\mathbf{x}}} \hat{\omega}\right\|_{L^{2}(\hat{T})}^{2} \quad \forall \omega \in H^{1}\left(T ; \mathbb{R}^{2}\right)
$$

where $\frac{B_{T} \hat{\omega}(\hat{\mathbf{x}})}{\operatorname{det}\left(B_{T}\right)}=\omega(\mathbf{x})$ for any $\hat{\mathbf{x}} \in \hat{T}$. If $T$ is a triangle for any $T \in \mathcal{T}_{h}$ and $h>0$, then the above inequality holds for any $h>0$.

Proof. We have,

$$
\begin{aligned}
& \operatorname{curl} \omega(\mathbf{x})=\frac{B_{T}}{\operatorname{det}\left(B_{T}\right)}(\operatorname{curl} \hat{\omega})(\hat{\mathbf{x}})=\frac{1}{\operatorname{det}\left(B_{T} D G_{T}(\hat{\mathbf{x}})\right)} B_{T} \operatorname{curl}_{\hat{\mathbf{x}}} \hat{\omega}(\hat{\mathbf{x}})\left(D G_{T}(\hat{\mathbf{x}})\right)^{\top} \\
& =\frac{\left(\operatorname{det}\left(B_{T}^{-1}\right)\right)^{2}}{\operatorname{det}\left(B_{T}^{-1} D G_{T}(\hat{\mathbf{x}})\right)} B_{T} \operatorname{curl}_{\hat{\mathbf{x}}} \hat{\omega}(\hat{\mathbf{x}}) B_{T}^{\top}\left(D G_{T}(\hat{\mathbf{x}}) B_{T}^{-1}\right)^{\top} \text {. }
\end{aligned}
$$

and

$$
\begin{aligned}
& \|\operatorname{curl} \omega\|_{L^{2}(T)}^{2}=\int_{T} \frac{\left(\operatorname{det}\left(B_{T}^{-1}\right)\right)^{4}}{\left(\operatorname{det}\left(B_{T}^{-1} D G_{T}(\hat{\mathbf{x}})\right)\right)^{2}}\left(D G_{T}(\hat{\mathbf{x}}) B_{T}^{-1}\right) B_{T}\left(\operatorname{curl}_{\hat{\mathbf{x}}} \hat{\omega}(\hat{\mathbf{x}})\right)^{\top} \\
& {\left[B_{T}^{\top} B_{T}\right] \operatorname{curl}_{\hat{\mathbf{x}}} \hat{\boldsymbol{\omega}}(\hat{\mathbf{x}}) B_{T}^{\top}\left(D G_{T}(\hat{\mathbf{x}}) B_{T}^{-1}\right)^{\top} d \mathbf{x}} \\
& =\int_{\hat{T}} \frac{\left(\operatorname{det}\left(B_{T}^{-1}\right)\right)^{3}}{\left(\operatorname{det}\left(B_{T}^{-1} D G_{T}(\hat{\mathbf{x}})\right)\right)^{2}}\left(D G_{T}(\hat{\mathbf{x}}) B_{T}^{-1}\right) B_{T}\left(\operatorname{curl}_{\hat{\mathbf{x}}} \hat{\omega}(\hat{\mathbf{x}})\right)^{\top} \\
& {\left[B_{T}^{\top} B_{T}\right] \operatorname{curl}_{\hat{\mathbf{x}}} \hat{\omega}(\hat{\mathbf{x}}) B_{T}^{\top}\left(D G_{T}(\hat{\mathbf{x}}) B_{T}^{-1}\right)^{\top} d \hat{\mathbf{x}} .}
\end{aligned}
$$

Since $c_{h} \rightarrow 0$ as $h \rightarrow 0, \lim _{h \rightarrow 0} \sup _{T \in \mathcal{T}_{h}} \sup _{\hat{\mathbf{x}} \in \hat{T}}\left\|D G_{T}(\hat{\mathbf{x}}) B_{T}^{-1}-I\right\|=0$.

By Lemma 3.9, $\lim _{h \rightarrow 0} \sup _{T \in \mathcal{T}_{h}} \sup _{\hat{\mathbf{x}} \in \hat{T}}\left|\left(\operatorname{det}\left(B_{T}^{-1} D G_{T}(\hat{\mathbf{x}})\right)\right)^{2}-1\right|=0$.

Since $\left(\mathcal{T}_{h}\right)_{h}$ is regular, there exists a constant $\sigma>0$ such that $\sigma_{1} / \sigma_{2} \leq \sigma$ for any $T \in \mathcal{T}_{h}$, with $\sigma_{1} \geq \sigma_{2}$ denoting the singular values of matrix $B_{T}$. Then $\left\|B_{T}^{\top}\right\|=\left\|B_{T}\right\|=\sigma_{1}$ and $\operatorname{det}\left(B_{T}^{-1}\right)=\sigma_{1}^{-1} \cdot \sigma_{2}^{-1}$. So $\left\|B_{T}^{\top}\right\|^{2} \cdot\left\|B_{T}\right\|^{2}\left(\operatorname{det}\left(B_{T}^{-1}\right)\right)^{3}=$ $\sigma_{1} / \sigma_{2}^{3} \leq \sigma / \sigma_{2}^{2} \leq c^{\prime} \tilde{h}_{T}^{-2}$ for some constant $c^{\prime}>0$.

Consequently, there exist $\delta_{1}>0$ and $C>0$ such that, for any $h<\delta_{1}$ and $T \in \mathcal{T}_{h}$,

$$
\begin{aligned}
& \int_{\hat{T}} \frac{\left(\operatorname{det}\left(B_{T}^{-1}\right)\right)^{3}}{\left(\operatorname{det}\left(B_{T}^{-1} D G_{T}(\hat{\mathbf{x}})\right)\right)^{2}}\left(D G_{T}(\hat{\mathbf{x}}) B_{T}^{-1}\right) B_{T}\left(\operatorname{curl}_{\hat{\mathbf{x}}} \hat{\omega}(\hat{\mathbf{x}})\right)^{\top} \\
& {\left[B_{T}^{\top} B_{T}\right] \operatorname{curl}_{\hat{\mathbf{x}}} \hat{\omega}(\hat{\mathbf{x}}) B_{T}^{\top}\left(D G_{T}(\hat{\mathbf{x}}) B_{T}^{-1}\right)^{\top} d \hat{\mathbf{x}} } \\
\leq & C \tilde{h}_{T}^{-2} \int_{\hat{T}}\left\|\left(\operatorname{curl}_{\hat{\mathbf{x}}} \hat{\omega}(\hat{\mathbf{x}})\right)^{\top}\left(\operatorname{curl}_{\hat{\mathbf{x}}} \hat{\omega}(\hat{\mathbf{x}})\right)\right\| d \hat{\mathbf{x}} .
\end{aligned}
$$


Again, it is easy to see that, if $T$ is a (regular) triangle for any $T \in \mathcal{T}_{h}$ and $h>0$, then the above inequality holds for any $h>0$. This finishes the proof.

Lemma 3.16. There exist $\delta>0$ and $C>0$ such that, for any $h<\delta$ and $T \in \mathcal{T}_{h}$,

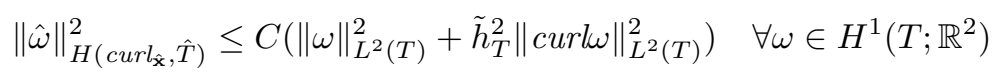

where $\frac{B_{T} \hat{\omega}(\hat{\mathbf{x}})}{\operatorname{det}\left(B_{T}\right)}=\omega(\mathbf{x})$ for any $\hat{\mathbf{x}} \in \hat{T}$. If $T$ is a triangle for any $T \in \mathcal{T}_{h}$ and $h>0$, then the above inequality holds for any $h>0$.

Proof. We have,

$$
\|\hat{\omega}\|_{H\left(\operatorname{curl}_{\hat{\mathbf{x}}, \hat{T})}\right.}^{2}=\|\hat{\omega}\|_{L^{2}(\hat{T})}^{2}+\| \operatorname{curl}_{\hat{\mathbf{x}} \hat{\omega} \|_{L^{2}(\hat{T})}^{2}}
$$

and

$$
\begin{aligned}
\|\hat{\omega}\|_{L^{2}(\hat{T})}^{2} & =\int_{\hat{T}}\left(\operatorname{det}\left(B_{T}\right)\right)^{2} \omega(\mathbf{x})^{\top} B_{T}^{-\top} B_{T}^{-1} \omega(\mathbf{x}) d \hat{\mathbf{x}} \\
& =\int_{T} \operatorname{det}\left(B_{T}\right) \operatorname{det}\left(B_{T} D G_{T}(\hat{\mathbf{x}})^{-1}\right) \omega(\mathbf{x})^{\top} B_{T}^{-\top} B_{T}^{-1} \omega(\mathbf{x}) d \mathbf{x} .
\end{aligned}
$$

By Lemma 3.9, $\lim _{h \rightarrow 0} \sup _{T \in \mathcal{T}_{h}} \sup _{\hat{\mathbf{x}} \in \hat{T}}\left|\operatorname{det}\left(B_{T} D G_{T}(\hat{\mathbf{x}})^{-1}\right)-1\right|=0$.

Since $\left(\mathcal{T}_{h}\right)_{h}$ is regular, there exists a constant $\sigma>0$ such that $\sigma_{1} / \sigma_{2} \leq \sigma$ for any $T \in \mathcal{T}_{h}$, where $\sigma_{1} \geq \sigma_{2}$ are the singular values of matrix $B_{T}$. Then $\left\|B_{T}^{-\top}\right\|=$ $\left\|B_{T}^{-1}\right\|=\sigma_{2}^{-1}$ and $\operatorname{det}\left(B_{T}\right)=\sigma_{1} \cdot \sigma_{2}$. So, $\left\|B_{T}^{-\top}\right\| \cdot\left\|B_{T}^{-1}\right\| \operatorname{det}\left(B_{T}\right)=\sigma_{1} / \sigma_{2} \leq \sigma$. Consequently, there exist $\delta_{1}>0$ and $C_{1}>0$ such that, for any $h<\delta_{1}$ and $T \in \mathcal{T}_{h}$,

$$
\operatorname{det}\left(B_{T}\right) \operatorname{det}\left(B_{T} D G_{T}(\hat{\mathbf{x}})^{-1}\right) \omega(\mathbf{x})^{\top} B_{T}^{-\top} B_{T}^{-1} \omega(\mathbf{x}) \leq C_{1}^{2} \omega(\mathbf{x})^{\top} \omega(\mathbf{x})
$$

for any $\omega \in H^{1}\left(T ; \mathbb{R}^{2}\right), \mathbf{x} \in T$. We can conclude that, for any $h<\delta_{1}$ and $T \in \mathcal{T}_{h}$,

$$
\|\hat{\omega}\|_{L^{2}(\hat{T})} \leq C_{1}\|\omega\|_{L^{2}(T)}, \forall \omega \in H^{1}\left(T ; \mathbb{R}^{2}\right) .
$$

At the same time,

$$
\begin{aligned}
\operatorname{curl}_{\hat{\mathbf{x}}} \hat{\omega}(\hat{\mathbf{x}}) & =\operatorname{det}\left(B_{T}\right) B_{T}^{-1} \operatorname{curl}_{\hat{\mathbf{x}}} \omega(\mathbf{x}) \\
& =\operatorname{det}\left(B_{T}\right) B_{T}^{-1} \operatorname{curl}_{\mathbf{x}} \omega(\mathbf{x})\left(D G_{T}(\hat{\mathbf{x}})\right)^{-\top} \operatorname{det}\left(D G_{T}(\hat{\mathbf{x}})\right) \\
& =\operatorname{det}\left(B_{T}\right) B_{T}^{-1} \operatorname{curl}_{\mathbf{x}} \omega(\mathbf{x}) B_{T}^{-\top}\left(B_{T}^{\top} D G_{T}(\hat{\mathbf{x}})^{-\top}\right) \operatorname{det}\left(D G_{T}(\hat{\mathbf{x}})\right) \\
& =\operatorname{det}\left(B_{T}\right) B_{T}^{-1} \operatorname{curl}_{\mathbf{x}} \omega(\mathbf{x}) B_{T}^{-\top}\left(D G_{T}(\hat{\mathbf{x}})^{-1} B_{T}\right)^{\top} \operatorname{det}\left(D G_{T}(\hat{\mathbf{x}})\right) .
\end{aligned}
$$

and

$$
\begin{aligned}
\left\|\operatorname{curl}_{\hat{\mathbf{x}}} \hat{\omega}\right\|_{L^{2}(\hat{T})}^{2}= & \int_{\hat{T}} \operatorname{det}\left(B_{T}\right)^{2} \operatorname{det}\left(D G_{T}(\hat{\mathbf{x}})\right)^{2}\left(D G_{T}(\hat{\mathbf{x}})^{-1} B_{T}\right) B_{T}^{-1}\left(\operatorname{curl}_{\mathbf{x}} \omega(\mathbf{x})\right)^{\top} \\
= & \int_{\hat{T}} \operatorname{det}\left(B_{T}^{-\top} B_{T}^{-1} \operatorname{curl} \operatorname{det}\left(D G_{T}(\hat{\mathbf{x}})\right)\left(D G_{T}^{-\top}(\hat{\mathbf{x}})^{-1} B_{T}\right) B_{T}^{-1}\left(\operatorname{curl}_{\mathbf{x}} \omega(\mathbf{x})\right)^{\top}\right. \\
& B_{T}^{-\top} B_{T}^{-1} \operatorname{curl}_{\mathbf{x}} \omega(\mathbf{x}) B_{T}^{-\top}\left(D G_{T}(\hat{\mathbf{x}})^{-1} B_{T}\right)^{\top} d \mathbf{x}
\end{aligned}
$$

According to Lemma 3.8, $\lim _{h \rightarrow 0} \sup _{T \in \mathcal{T}_{h}} \sup _{\hat{\mathbf{x}} \in \hat{T}}\left\|D G_{T}(\hat{\mathbf{x}})^{-1} B_{T}-I\right\|=0$.

By Lemma 3.9, $\lim _{h \rightarrow 0} \sup _{T \in \mathcal{T}_{h}} \sup _{\hat{\mathbf{x}} \in \hat{T}}\left|\operatorname{det}\left(B_{T}^{-1} D G_{T}(\hat{\mathbf{x}})\right)-1\right|=0$. 
Since $\left(\mathcal{T}_{h}\right)_{h}$ is regular, $\operatorname{det}\left(B_{T}\right)^{3}\left\|B_{T}^{-\top}\right\|^{2} \cdot\left\|B_{T}^{-1}\right\|^{2} \leq \sigma \tilde{h}_{T}$, for some constant $\sigma>0$.

There exist thus $\delta_{2}>0$ and $C_{2}>0$ such that, for any $h<\delta_{2}$ and $T \in \mathcal{T}_{h}$,

$$
\begin{aligned}
& \operatorname{det}\left(B_{T}\right)^{3} \operatorname{det}\left(B_{T}^{-1} D G_{T}(\hat{\mathbf{x}})\right)\left(D G_{T}(\hat{\mathbf{x}})^{-1} B_{T}\right) B_{T}^{-1}\left(\operatorname{curl}_{\mathbf{x}} \omega(\mathbf{x})\right)^{\top} \\
& B_{T}^{-\top} B_{T}^{-1} \operatorname{curl}_{\mathbf{x}} \omega(\mathbf{x}) B_{T}^{-\top}\left(D G_{T}(\hat{\mathbf{x}})^{-1} B_{T}\right)^{\top} d \mathbf{x} \\
\leq & C_{2}^{2} \tilde{h}_{T}^{2}\left\|\left(\operatorname{curl}_{\mathbf{x}} \omega(\mathbf{x})\right)^{\top} \operatorname{curl}_{\mathbf{x}} \omega(\mathbf{x})\right\| \quad \forall \omega \in H^{1}\left(T ; \mathbb{R}^{2}\right), \mathbf{x} \in T .
\end{aligned}
$$

We conclude that, for any $h<\delta_{2}$ and $T \in \mathcal{T}_{h}$,

$$
\left\|\operatorname{curl}_{\hat{\mathbf{x}}} \hat{\omega}\right\|_{L^{2}(\hat{T})} \leq C_{2} \tilde{h}_{T}\|\operatorname{curl} \omega\|_{L^{2}(T)}, \forall \omega \in H^{1}\left(T ; \mathbb{R}^{2}\right) .
$$

Again, it is easy to see that, if $T$ is a triangle for any $T \in \mathcal{T}_{h}$ and $h>0$, then the inequality above holds for any $h>0$. This ends the proof.

Lemma 3.17. Let $T$ be a curved triangle. Let $u(\mathbf{x})$ be defined on $T$ and $\hat{u}(\hat{\mathbf{x}})$ be defined on $\hat{T}$ and

$$
u=\hat{u} \circ G_{T}^{-1} \quad \text { or } \quad \hat{u}=u \circ G_{T}
$$

where $G_{T}$ is the element map. Then $u(\mathbf{x}) \in L^{2}(T)$ if and only if $\hat{u}(\hat{x}) \in L^{2}(\hat{T})$.

Proof. This is an immediate consequence of the fact that $G_{T}$ is a $C^{1}$-diffeomorphism.

\section{Finite Element sPaCes}

We begin by introducing the relevant finite element spaces on the reference triangle. Then, for any curved triangle $T$, we define the corresponding finite element spaces on $T$ by the pull back mappings associated with the inverse of $G_{T}$. Finally, we define the finite element spaces on a whole mesh $\mathcal{T}_{h}$ by "gluing" the finite element spaces on curved triangles.

4.1. Finite element spaces on the reference triangle. For any $r \in \mathbb{Z}_{+}:=$ $\{n \in \mathbb{Z}: n \geq 0\}$, we introduce

$$
\begin{aligned}
& \mathcal{P}_{r}(\hat{T}):=\{\text { space of polynomials of order } r \text { on } \hat{T}\}, \\
& \mathcal{P}_{r} \Lambda^{0}(\hat{T})=\mathcal{P}_{r} \Lambda^{2}(\hat{T}):=\mathcal{P}_{r}(\hat{T}), \mathcal{P}_{r} \Lambda^{1}(\hat{T}):=\left[\mathcal{P}_{r}(\hat{T})\right]^{2}, \\
& \stackrel{\mathcal{P}}{r}_{r} \Lambda^{0}(\hat{T}):=\left\{\hat{w} \in \mathcal{P}_{r} \Lambda^{0}(\hat{T}):\left.\hat{w}\right|_{\hat{e}}=0, \forall \hat{e} \in \triangle_{1}(\hat{T})\right\}, \\
& \mathcal{P}_{r}^{-} \Lambda^{1}(\hat{T}):=\left[\mathcal{P}_{r-1}(\hat{T})\right]^{2}+\left(\hat{x}_{1}, \hat{x}_{2}\right)^{\top} \mathcal{P}_{r-1}(\hat{T}), \\
& \mathcal{P}_{r} \Lambda^{0}\left(\hat{T} ; \mathbb{R}^{2}\right)=\mathcal{P}_{r} \Lambda^{2}\left(\hat{T} ; \mathbb{R}^{2}\right):=\left[\mathcal{P}_{r}(\hat{T})\right]^{2}, \\
& \mathcal{P}_{r} \Lambda^{1}\left(\hat{T} ; \mathbb{R}^{2}\right):=\left\{\left[\begin{array}{cc}
\sigma_{11} & \sigma_{12} \\
\sigma_{21} & \sigma_{22}
\end{array}\right]:\left(\sigma_{11}, \sigma_{12}\right)^{\top},\left(\sigma_{21}, \sigma_{22}\right)^{\top} \in \mathcal{P}_{r} \Lambda^{1}(\hat{T})\right\} .
\end{aligned}
$$

In $[5,14]$, spaces in $(4.1)$ are defined in the language of exterior calculus. Here we just rewrite them using the language of calculus. We refer to [5] and [14] for a detailed correspondence before the exterior and classical calculus notations.

We denote by $\tilde{r}$ a mapping from $\triangle(\hat{T})$ to $\mathbb{Z}_{+}$such that if $\hat{e}, \hat{f} \in \Delta(T)$ and $\hat{e} \subset \hat{f}$ then $\tilde{r}(\hat{e}) \leq \tilde{r}(\hat{f})$. We introduce now formally the FE spaces of variable order. 


\section{Definition 4.1.}

$$
\begin{aligned}
\mathcal{P}_{\tilde{r}} \Lambda^{0}(\hat{T}):= & \left\{\hat{u} \in \mathcal{P}_{\tilde{r}(\hat{T})} \Lambda^{0}(\hat{T}): \forall \hat{e} \in \triangle_{1}(\hat{T}),\left.\hat{u}\right|_{\hat{e}} \in \mathcal{P}_{\tilde{r}(\hat{e})}(\hat{e})\right\}, \\
& \mathcal{P}_{\tilde{r}} \Lambda^{2}(\hat{T}):=\mathcal{P}_{\tilde{r}(\hat{T})} \Lambda^{2}(\hat{T})=\mathcal{P}_{\tilde{r}(\hat{T})}(\hat{T}), \\
\mathcal{P}_{\tilde{r}} \Lambda^{1}(\hat{T}):= & \left\{\hat{\omega} \in \mathcal{P}_{\tilde{r}(\hat{T})} \Lambda^{1}(\hat{T}): \forall \hat{e} \in \triangle_{1}(\hat{T}),\left.\hat{\omega} \cdot \hat{\mathbf{n}}\right|_{\hat{e}} \in \mathcal{P}_{\tilde{r}(\hat{e})}(\hat{e})\right\}, \\
\mathcal{P}_{\tilde{r}}^{-} \Lambda^{1}(\hat{T}):= & \left\{\hat{\omega} \in \mathcal{P}_{\tilde{r}(\hat{T})}^{-} \Lambda^{1}(\hat{T}): \forall \hat{e} \in \triangle_{1}(\hat{T}), \hat{\omega} \cdot \hat{\mathbf{n}} \mid \hat{e} \in \mathcal{P}_{\tilde{r}(\hat{e})-1}(\hat{e})\right\}, \\
& \mathcal{P}_{\tilde{r}} \Lambda^{0}\left(\hat{T} ; \mathbb{R}^{2}\right)=\mathcal{P}_{\tilde{r}} \Lambda^{2}\left(\hat{T} ; \mathbb{R}^{2}\right):=\left[\mathcal{P}_{\tilde{r}}(\hat{T})\right]^{2}, \\
\mathcal{P}_{\tilde{r}} \Lambda^{1}\left(\hat{T} ; \mathbb{R}^{2}\right):= & \left\{\left[\begin{array}{ll}
\sigma_{11} & \sigma_{12} \\
\sigma_{21} & \sigma_{22}
\end{array}\right]:\left(\sigma_{11}, \sigma_{12}\right)^{\top},\left(\sigma_{21}, \sigma_{22}\right)^{\top} \in \mathcal{P}_{\tilde{r}} \Lambda^{1}(\hat{T})\right\} .
\end{aligned}
$$

Here $\hat{\mathbf{n}}$ is the outward normal unit vector along $\partial \hat{T}$.

Remark 4.2. According to [5], for any $\hat{e} \in \triangle_{1}(\hat{T})$, we have

$$
\left.\mathcal{P}_{\tilde{r}} \Lambda^{0}(\hat{T})\right|_{\hat{e}}=\mathcal{P}_{\tilde{r}(\hat{e})}(\hat{e}),\left.\mathcal{P}_{\tilde{r}} \Lambda^{1}(\hat{T})\right|_{\hat{e}} \cdot \hat{\mathbf{n}}=\mathcal{P}_{\tilde{r}(\hat{e})}(\hat{e}),\left.\mathcal{P}_{\tilde{r}}^{-} \Lambda^{1}(\hat{T})\right|_{\hat{e}} \cdot \hat{\mathbf{n}}=\mathcal{P}_{\tilde{r}(\hat{e})-1}(\hat{e}) .
$$

\section{Lemma 4.3.}

$$
\begin{aligned}
& \mathcal{P}_{\tilde{r}} \Lambda^{1}(\hat{T}) \subset \mathcal{P}_{\tilde{r}+1}^{-} \Lambda^{1}(\hat{T}) \subset \mathcal{P}_{\tilde{r}+1} \Lambda^{1}(\hat{T}), \\
& \operatorname{div}_{\hat{\mathbf{x}}} \mathcal{P}_{\tilde{r}+1} \Lambda^{1}(\hat{T}) \subset \mathcal{P}_{\tilde{r}} \Lambda^{2}(\hat{T}), \operatorname{curl}_{\hat{\mathbf{x}}} \mathcal{P}_{\tilde{r}+1} \Lambda^{0}(\hat{T}) \subset \mathcal{P}_{\tilde{r}} \Lambda^{1}(\hat{T}) .
\end{aligned}
$$

Proof. The inclusions are a straightforward consequence of Definition 4.1.

4.2. Finite element spaces on a curved triangle. Let $T$ be a curved triangle from Definition 3.1 with $G_{T}$ denoting the corresponding $C^{1}$-diffeomorphism from $\hat{T}$ to $T, \mathbf{x}=G_{T}(\hat{\mathbf{x}})$. We begin by introducing formally the mapping $\tilde{r}$ from $\triangle(T)$ to $\mathbb{Z}_{+}$specifying the local order of discretization.

Definition 4.4. We denote by $\tilde{r}$ a mapping from $\triangle(T)$ to $\mathbb{Z}_{+}$such that if $e, f \in$ $\triangle(T)$ and $e \subset f$ then $\tilde{r}(e) \leq \tilde{r}(f)$. With the same symbol $\tilde{r}$ we denote the corresponding mapping from $\triangle(\hat{T})$ to $\mathbb{Z}, \tilde{r}(\hat{f}):=\tilde{r}(f)$ for any $\hat{f} \in \triangle(\hat{T})$, where $f=G_{T}(\hat{f})$.

We define now the following $\mathrm{FE}$ spaces on $T$ :

\section{Definition 4.5.}

$$
\begin{aligned}
\mathcal{P}_{\tilde{r}} \Lambda^{0}(T) & :=\left\{u(\mathbf{x}): \hat{u}(\hat{\mathbf{x}}) \in \mathcal{P}_{\tilde{r}} \Lambda^{0}(\hat{T}) \text { where } u(\mathbf{x})=\hat{u}(\hat{\mathbf{x}})\right\}, \\
\mathcal{P}_{\tilde{r}} \Lambda^{1}(T) & :=\left\{\omega(\mathbf{x}): \hat{\omega}(\hat{\mathbf{x}}) \in \mathcal{P}_{\tilde{r}} \Lambda^{1}(\hat{T}) \text { where } \omega(\mathbf{x})=\frac{1}{\operatorname{det}\left(D G_{T}(\hat{\mathbf{x}})\right)} D G_{T}(\hat{\mathbf{x}}) \hat{\omega}(\hat{\mathbf{x}})\right\}, \\
\mathcal{P}_{\tilde{r}}^{-} \Lambda^{1}(T) & :=\left\{\omega(\mathbf{x}): \hat{\omega}(\hat{\mathbf{x}}) \in \mathcal{P}_{\tilde{r}}^{-} \Lambda^{1}(\hat{T}) \text { where } \omega(\mathbf{x})=\frac{1}{\operatorname{det}\left(D G_{T}(\hat{\mathbf{x}})\right)} D G_{T}(\hat{\mathbf{x}}) \hat{\omega}(\hat{\mathbf{x}})\right\}, \\
\mathcal{P}_{\tilde{r}} \Lambda^{2}(T) & :=\left\{u(\mathbf{x}): \hat{u}(\hat{\mathbf{x}}) \in \mathcal{P}_{\tilde{r}} \Lambda^{2}(\hat{T}) \text { where } u(\mathbf{x})=\frac{1}{\operatorname{det}\left(D G_{T}(\hat{\mathbf{x}})\right)} \hat{u}(\hat{\mathbf{x}})\right\}, \\
\mathcal{P}_{\tilde{r}} \Lambda^{0}\left(T ; \mathbb{R}^{2}\right) & :=\left\{\left(u_{1}, u_{2}\right): u_{1}, u_{2} \in \mathcal{P}_{\tilde{r}} \Lambda^{0}(T)\right\}, \\
\mathcal{P}_{\tilde{r}} \Lambda^{2}\left(T ; \mathbb{R}^{2}\right) & :=\left\{\left(u_{1}, u_{2}\right): u_{1}, u_{2} \in \mathcal{P}_{\tilde{r}} \Lambda^{2}(T)\right\}, \\
\mathcal{P}_{\tilde{r}} \Lambda^{1}\left(T ; \mathbb{R}^{2}\right) & :=\left\{\left[\begin{array}{ll}
\sigma_{11} & \sigma_{12} \\
\sigma_{21} & \sigma_{22}
\end{array}\right]:\left(\sigma_{11}, \sigma_{12}\right)^{\top},\left(\sigma_{21}, \sigma_{22}\right)^{\top} \in \mathcal{P}_{\tilde{r}} \Lambda^{1}(T)\right\} .
\end{aligned}
$$


Remark 4.6. Since $G_{T}: \hat{T} \rightarrow T$ is a $C^{1}$-diffeomorphism with $\operatorname{det}\left(D G_{T}(\hat{\mathbf{x}})\right) \neq 0$, for any $\hat{\mathbf{x}} \in \hat{T}$, the formulae in Definition 4.5 are well-defined. The mappings used in the definition are the standard pull back mappings for differential forms $\Lambda^{0}, \Lambda^{1}, \Lambda^{2}$, see e.g. formulas $(2.24),(2.26),(2.27)$ in [13].

Lemma 4.7. For any edge $e \in \triangle_{1}(T)$, let $\zeta(s)$ be the local parameterization for $e$ discussed in Definition 3.3, i.e. the affine mapping from the reference interval onto $\hat{e} \in \triangle_{1}(\hat{T})$. We have then,

$$
\begin{aligned}
\left.\mathcal{P}_{\tilde{r}} \Lambda^{0}(T)\right|_{e} & =\left\{u(\mathbf{x}) \text { where } \mathbf{x} \in e: u\left(G_{T}(\zeta(s))\right) \in \mathcal{P}_{\tilde{r}(\hat{e})}(\hat{e})\right\}, \\
\left.\mathcal{P}_{\tilde{r}} \Lambda^{1}(T) \cdot \mathbf{n}\right|_{e} & =\left\{u(\mathbf{x}) \text { where } \mathbf{x} \in e: u\left(G_{T}(\zeta(s))\right)\left\|D\left(G_{T} \circ \zeta\right)(s)\right\| \in \mathcal{P}_{\tilde{r}(\hat{e})}(\hat{e})\right\}, \\
\left.\mathcal{P}_{\tilde{r}}^{-} \Lambda^{1}(T) \cdot \mathbf{n}\right|_{e} & =\left\{u(\mathbf{x}) \text { where } \mathbf{x} \in e: u\left(G_{T}(\zeta(s))\right)\left\|D\left(G_{T} \circ \zeta\right)(s)\right\| \in \mathcal{P}_{\tilde{r}(\hat{e})-1}(\hat{e})\right\} .
\end{aligned}
$$

In addition, the above equalities do not depend on the choice of the orientation of the local parameterization $\zeta(s)$.

Proof. These are trivial observations on pull back mappings and their restrictions to edges.

\section{Lemma 4.8 .}

$$
\begin{aligned}
& \mathcal{P}_{\tilde{r}} \Lambda^{1}(T) \subset \mathcal{P}_{\tilde{r}+1}^{-} \Lambda^{1}(T) \subset \mathcal{P}_{\tilde{r}+1} \Lambda^{1}(T), \\
& \operatorname{div}_{\tilde{r}+1} \Lambda^{1}(T) \subset \mathcal{P}_{\tilde{r}} \Lambda^{2}(T), \operatorname{curl}_{\tilde{r}+1} \Lambda^{0}(T) \subset \mathcal{P}_{\tilde{r}} \Lambda^{1}(T)
\end{aligned}
$$

Proof. The embeddings are a straightforward consequence of Definition 4.5, Lemma 4.3, and the commutativity of pull back mappings with exterior derivatives (curl, div).

4.3. Finite element spaces on a $C^{0}$-compatible mesh. Let $\mathcal{T}_{h}$ be a $C^{0}$-compatible mesh from Definition 3.3. We extend the $\tilde{r}$ mapping to a global map defined on $\triangle\left(\mathcal{T}_{h}\right)$ with values in $\mathbb{Z}_{+}$such that if $e \subset f$, then $\tilde{r}(e) \leq \tilde{r}(f)$.

Definition 4.9. We put $\Omega_{h}:=\bigcup_{T \in \mathcal{T}_{h}} T$.

$C \Lambda^{0}\left(\mathcal{T}_{h}\right):=\left\{u \in H^{1}\left(\Omega_{h}\right): u\right.$ is piece-wise smooth with respect to $\left.\mathcal{T}_{h}\right\}$,

$C \Lambda^{1}\left(\mathcal{T}_{h}\right):=\left\{\omega \in H\left(\operatorname{div}, \Omega_{h}\right): \omega\right.$ is piece-wise smooth with respect to $\left.\mathcal{T}_{h}\right\}$,

$C \Lambda^{2}\left(\mathcal{T}_{h}\right):=\left\{u \in L^{2}\left(\Omega_{h}\right): u\right.$ is piece-wise smooth with respect to $\left.\mathcal{T}_{h}\right\}$.

We define

$$
\begin{aligned}
\mathcal{P}_{\tilde{r}} \Lambda^{0}\left(\mathcal{T}_{h}\right) & :=\left\{u \in C \Lambda^{0}\left(\mathcal{T}_{h}\right):\left.u\right|_{T} \in \mathcal{P}_{\tilde{r}} \Lambda^{0}(T), \forall T \in \mathcal{T}_{h}\right\}, \\
\mathcal{P}_{\tilde{r}} \Lambda^{1}\left(\mathcal{T}_{h}\right) & :=\left\{\omega \in C \Lambda^{1}\left(\mathcal{T}_{h}\right):\left.\omega\right|_{T} \in \mathcal{P}_{\tilde{r}} \Lambda^{1}(T), \forall T \in \mathcal{T}_{h}\right\}, \\
\mathcal{P}_{\tilde{r}}^{-} \Lambda^{1}\left(\mathcal{T}_{h}\right) & :=\left\{\omega \in C \Lambda^{1}\left(\mathcal{T}_{h}\right):\left.\omega\right|_{T} \in \mathcal{P}_{\tilde{r}}^{-} \Lambda^{1}(T), \forall T \in \mathcal{T}_{h}\right\}, \\
\mathcal{P}_{\tilde{r}} \Lambda^{2}\left(\mathcal{T}_{h}\right) & :=\left\{u \in C \Lambda^{2}\left(\mathcal{T}_{h}\right):\left.u\right|_{T} \in \mathcal{P}_{\tilde{r}} \Lambda^{2}(T), \forall T \in \mathcal{T}_{h}\right\}, \\
\mathcal{P}_{\tilde{r}} \Lambda^{0}\left(\mathcal{T}_{h} ; \mathbb{R}^{2}\right) & :=\left[\mathcal{P}_{\tilde{r}} \Lambda^{0}\left(\mathcal{T}_{h}\right)\right]^{2}, \mathcal{P}_{\tilde{r}} \Lambda^{2}\left(\mathcal{T}_{h} ; \mathbb{R}^{2}\right):=\left[\mathcal{P}_{\tilde{r}} \Lambda^{2}\left(\mathcal{T}_{h}\right)\right]^{2}, \\
\mathcal{P}_{\tilde{r}} \Lambda^{1}\left(\mathcal{T}_{h} ; \mathbb{R}^{2}\right) & :=\left\{\left[\begin{array}{cc}
\sigma_{11} & \sigma_{12} \\
\sigma_{21} & \sigma_{22}
\end{array}\right]:\left(\sigma_{11}, \sigma_{12}\right)^{\top},\left(\sigma_{21}, \sigma_{22}\right)^{\top} \in \mathcal{P}_{\tilde{r}} \Lambda^{1}\left(\mathcal{T}_{h}, \mathbb{R}^{2}\right)\right\} .
\end{aligned}
$$

Remark 4.10. According to Lemma 4.7 and the fact that $\mathcal{T}_{h}$ is $C^{0}$-compatible, we can conclude that $\left.\mathcal{P}_{\tilde{r}} \Lambda^{0}\left(\mathcal{T}_{h}\right)\right|_{T}=\mathcal{P}_{\tilde{r}} \Lambda^{0}(T),\left.\mathcal{P}_{\tilde{r}} \Lambda^{1}\left(\mathcal{T}_{h}\right)\right|_{T}=\mathcal{P}_{\tilde{r}} \Lambda^{1}(T),\left.\mathcal{P}_{\tilde{r}}^{-} \Lambda^{1}\left(\mathcal{T}_{h}\right)\right|_{T}=$ $\mathcal{P}_{\tilde{r}}^{-} \Lambda^{1}(T),\left.\mathcal{P}_{\tilde{r}} \Lambda^{2}\left(\mathcal{T}_{h}\right)\right|_{T}=\mathcal{P}_{\tilde{r}} \Lambda^{2}(T)$, for any $T \in \mathcal{T}_{h}$. For standard (not curved) 
triangulations $\mathcal{T}_{h}$, spaces $\mathcal{P}_{\tilde{r}} \Lambda^{0}\left(\mathcal{T}_{h}\right), \mathcal{P}_{\tilde{r}} \Lambda^{1}\left(\mathcal{T}_{h}\right), \mathcal{P}_{\tilde{r}}^{-} \Lambda^{1}\left(\mathcal{T}_{h}\right), \mathcal{P}_{\tilde{r}} \Lambda^{2}\left(\mathcal{T}_{h}\right)$ coincide with those analyzed in [19].

Lemma 4.11.

$$
\begin{aligned}
& \mathcal{P}_{\tilde{r}} \Lambda^{1}\left(\mathcal{T}_{h}\right) \subset \mathcal{P}_{\tilde{r}+1}^{-} \Lambda^{1}\left(\mathcal{T}_{h}\right) \subset \mathcal{P}_{\tilde{r}+1} \Lambda^{1}\left(\mathcal{T}_{h}\right), \\
& \operatorname{div} \mathcal{P}_{\tilde{r}+1} \Lambda^{1}\left(\mathcal{T}_{h}\right) \subset \mathcal{P}_{\tilde{r}} \Lambda^{2}\left(\mathcal{T}_{h}\right), \operatorname{curl}_{\tilde{r}+1} \Lambda^{0}\left(\mathcal{T}_{h}\right) \subset \mathcal{P}_{\tilde{r}} \Lambda^{1}\left(\mathcal{T}_{h}\right) .
\end{aligned}
$$

Proof. This is an immediate consequence of Lemma 4.8 .

\section{MiXed FORMULATION FOR ELASTICITY WITH WEAKLY IMPOSED SYMMETRY}

We assume that there is $r_{\max } \in \mathbb{N}$ such that, for any $h>0$ and $f \in \Delta\left(\mathcal{T}_{h}\right)$, $\tilde{r}(f) \leq r_{\max }$.

We recall the mixed formulation (1.8): Find $(\sigma, u, p) \in H(\operatorname{div}, \Omega ; \mathbb{M}) \times L^{2}\left(\Omega ; \mathbb{R}^{2}\right) \times$ $L^{2}(\Omega)$ such that

$$
\begin{aligned}
\langle A \sigma, \tau\rangle+\langle\operatorname{div} \tau, u\rangle-\left\langle S_{1} \tau, p\right\rangle & =0, \quad \tau \in H(\operatorname{div}, \Omega ; \mathbb{M}), \\
\langle\operatorname{div} \sigma, v\rangle & =\langle f, v\rangle, \quad v \in L^{2}\left(\Omega ; \mathbb{R}^{2}\right), \\
\left\langle S_{1} \sigma, q\right\rangle & =0, \quad q \in L^{2}(\Omega) .
\end{aligned}
$$

Here $\langle\cdot, \cdot\rangle$ is the standard $L^{2}$ inner product on $\Omega$. This problem is well-posed. See [5] and [14] for the proof.

We consider now a finite element discretization of (5.1). For this, we choose families of finite-dimensional subspaces

$$
\Lambda_{h}^{1}(\mathbb{M}) \subset H(\operatorname{div}, \Omega ; \mathbb{M}), \Lambda_{h}^{2}\left(\mathbb{R}^{2}\right) \subset L^{2}\left(\Omega ; \mathbb{R}^{2}\right), \Lambda_{h}^{2} \subset L^{2}(\Omega),
$$

indexed by $h$, and seek a discrete solution $\left(\sigma_{h}, u_{h}, p_{h}\right) \in \Lambda_{h}^{1}(\mathbb{M}) \times \Lambda_{h}^{2}\left(\mathbb{R}^{2}\right) \times \Lambda_{h}^{2}$ such that

$$
\begin{aligned}
\left\langle A \sigma_{h}, \tau\right\rangle+\left\langle\operatorname{div} \tau, u_{h}\right\rangle-\left\langle S_{1} \tau, p_{h}\right\rangle & =0, \quad \tau \in \Lambda_{h}^{1}(\mathbb{M}), \\
\left\langle\operatorname{div} \sigma_{h}, v\right\rangle & =\langle f, v\rangle, \quad v \in \Lambda_{h}^{2}\left(\mathbb{R}^{2}\right), \\
\left\langle S_{1} \sigma_{h}, q\right\rangle & =0, \quad q \in \Lambda_{h}^{2} .
\end{aligned}
$$

The stability of (5.2) will be ensured by the Brezzi stability conditions:

$$
\begin{gathered}
(\mathrm{S} 1)\|\tau\|_{H(\operatorname{div}, \Omega ; \mathbb{M})}^{2} \leq c_{1}(A \tau, \tau) \text { whenever } \tau \in \Lambda_{h}^{1}\left(\mathbb{R}^{2}\right) \text { satisfies }\langle\operatorname{div} \tau, v\rangle=0 \\
\forall v \in \Lambda_{h}^{2}\left(\mathbb{R}^{2}\right) \text { and }\left\langle S_{1} \tau, q\right\rangle=0 \forall q \in \Lambda_{h}^{2},
\end{gathered}
$$

(S2) for all nonzero $(v, q) \in \Lambda_{h}^{2}\left(\mathbb{R}^{2}\right) \times \Lambda_{h}^{2}$, there exists nonzero

$$
\tau \in \Lambda_{h}^{1}\left(\mathbb{R}^{2}\right) \text { with }\langle\operatorname{div} \tau, v\rangle-\left\langle S_{1} \tau, q\right\rangle \geq c_{2}\|\tau\|_{H(\operatorname{div}, \Omega ; \mathbb{M})}\left(\|v\|_{L^{2}\left(\Omega ; \mathbb{R}^{2}\right)}+\|q\|_{L^{2}(\Omega)}\right),
$$

where constants $c_{1}$ and $c_{2}$ are independent of $h$.

For meshes of arbitrary but uniform order, conditions (5.3) and (5.4) have been proved in [5] and [14]. In what follows, we will demonstrate that they are also satisfied for (2D) meshes with elements of variable (but limited) order.

Before presenting our proof, we would like to comment shortly on difficulties encountered in proving stability for generalizing AFW elements with variable order. As we have shown in Section 3, it is rather straightforward to generalize AFW elements to the variable order case. The following commuting diagrams are essential in the stability proof from $[6,5,14]$, 


$$
\begin{aligned}
& \text { (5.5) }
\end{aligned}
$$

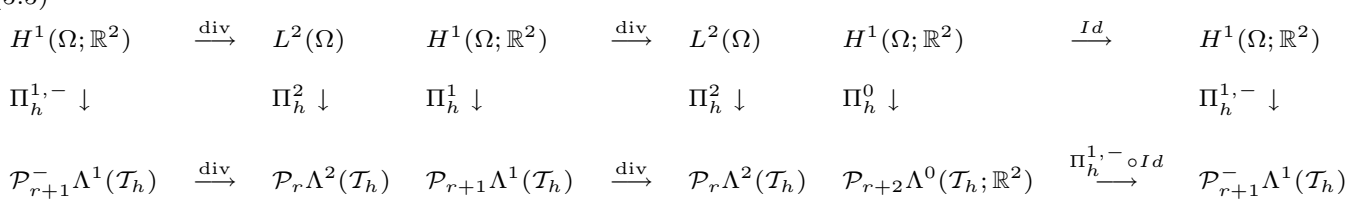

When uniform order $r$ is replaced by variable order $\tilde{r}$, then the left and middle diagrams do not commute if $\Pi_{h}^{1,-}$ and $\Pi_{h}^{1}$ are natural generalizations of canonical projection operators introduced in $[6,5,14]$. A counterexample is given in the appendix of [19]. To our best knowledge, the only operators which make these two diagrams commute for meshes with variable order, are projection based interpolation operators introduced in [19]. But then we need to construct an operator $\Pi_{h}^{0}$ that would make the right diagram commute if $\Pi_{h}^{1,-}$ is the projection based interpolation operator. In [19], we constructed such an operator $W_{h}$, which take the place of $\Pi_{h}^{0}$. But we were only able to prove its well-definedness for $0 \leq \tilde{r}(T) \leq 3$. In the following sections we pursue a different strategy, defining new projection based interpolation $\Pi_{h}^{1,-}, \Pi_{h}^{1}$ operators, and a new operator $W_{h}$ so that we can make all three diagrams commute.

\section{Preliminaries For the Proof of Stability}

We begin by recalling our assumptions on the domain and meshes: $\Omega$ is a (curvilinear) polygon and it is meshed with a family $\left(\mathcal{T}_{h}\right)_{h}$ of $C^{0}$-compatible meshes of class $C^{2}$. For any mesh $\mathcal{T}_{h}$, mapping $\tilde{r}: \triangle\left(\mathcal{T}_{h}\right) \rightarrow \mathbb{Z}_{+}$defines a locally variable order of discretization that satisfies the minimum rule, $\tilde{r}(e) \leq \tilde{r}(f)$ for $e \subset f, e, f \in \triangle_{T}$. The maximum order is limited, i.e. $\sup _{h} \sup _{T \in \mathcal{T}_{h}} \tilde{r}(T)<\infty$.

Definition 6.1. For any $T \in \mathcal{T}_{h}$, we define a linear operator $\Pi_{\tilde{r}, T}^{2}: L^{2}(T) \longrightarrow$ $\mathcal{P}_{\tilde{r}} \Lambda^{2}(T)$ by the relations

$$
\int_{T}\left(\Pi_{\tilde{r}, T}^{2} u(\mathbf{x})-u(\mathbf{x})\right) \hat{\psi}(\hat{\mathbf{x}}(\mathbf{x})) d \mathbf{x}=0 \quad \forall \hat{\psi} \in \mathcal{P}_{\tilde{r}(T)}(\hat{T})
$$

Above, $\hat{\mathbf{x}}(\mathbf{x})$ signifies the inverse of the element map $\mathbf{x}=G_{T}(\hat{\mathbf{x}})$.

Definition 6.2. Operator $\Pi_{\tilde{r}, \hat{T}}^{2}: L^{2}(\hat{T}) \longrightarrow \mathcal{P}_{\tilde{r}} \Lambda^{2}(\hat{T})$ will denote the $L^{2}$-projection in the reference space,

$$
\int_{T}\left(\Pi_{\tilde{r}, \hat{T}}^{2} \hat{u}(\hat{\mathbf{x}})-\hat{u}(\hat{\mathbf{x}})\right) \hat{\psi}(\hat{\mathbf{x}}) d \hat{\mathbf{x}}=0 \quad \forall \hat{\psi} \in \mathcal{P}_{\tilde{r}(T)}(\hat{T})
$$

Remark 6.3. Operator $\Pi_{\tilde{r}, T}^{2}$ is a weighted $L^{2}$-projection in the physical space. For a regular triangle (affine element map), the jacobian is constant, and $\Pi_{\tilde{r}, T}^{2}$ reduces to the standard $L^{2}$-projection in the physical space.

Lemma 6.4. For any $T \in \mathcal{T}_{h}$, and arbitrary $u(\mathbf{x}) \in L^{2}(T)$, we define $\hat{u}(\hat{\mathbf{x}})$ by the relation:

Then $\hat{u}(\hat{\mathbf{x}}) \in L^{2}(\hat{T})$, and

$$
u(\mathbf{x}(\hat{\mathbf{x}}))=\frac{\hat{u}(\hat{\mathbf{x}})}{\operatorname{det}\left(D G_{T}(\hat{\mathbf{x}})\right)}
$$

$$
\Pi_{\tilde{r}, T}^{2} u(\mathbf{x}(\hat{\mathbf{x}}))=\frac{\Pi_{\tilde{r}, \hat{T}}^{2} \hat{u}(\hat{\mathbf{x}})}{\left.\operatorname{det}\left(D G_{T}(\hat{\mathbf{x}})\right)\right)}
$$


Above, $\mathbf{x}(\hat{\mathbf{x}})$ signifies the element map $\mathbf{x}=G_{T}(\hat{\mathbf{x}})$.

Proof. Proof follows immediately from Lemma 3.17 and the definitions of the two projections.

Lemma 6.5. For any $\varepsilon>0$, there exists $\delta>0$ such that, for any $h<\delta$ and $T \in \mathcal{T}_{h}$,

$$
\left\|\Pi_{\tilde{r}, T}^{2} u-P_{\tilde{r}, T} u\right\|_{L^{2}(T)} \leq \varepsilon\|u\|_{L^{2}(T)}, \forall u \in L^{2}(T) .
$$

Here $P_{\tilde{r}, T}$ is the standard $L^{2}$-projection onto $\mathcal{P}_{\tilde{r}} \Lambda^{2}(T)$.

Proof. We put $r=\tilde{r}(T)$. We assume $\left\{\hat{\xi}_{1}(\hat{\mathbf{x}}), \cdots, \hat{\xi}(\hat{\mathbf{x}})_{l_{r}}\right\}$ is a basis for $\mathcal{P}_{\tilde{r}} \Lambda^{2}(\hat{T})$. Then

and

$$
\Pi_{\tilde{r}, T}^{2} u(\mathbf{x}(\hat{\mathbf{x}}))=\frac{1}{\operatorname{det}\left(D G_{T}(\hat{\mathbf{x}})\right)} \sum_{i=1}^{l_{r}} \alpha_{i} \hat{\xi}_{i}(\hat{\mathbf{x}})
$$

$$
P_{\tilde{r}, T}^{2} u(\mathbf{x}(\hat{\mathbf{x}}))=\frac{1}{\operatorname{det}\left(D G_{T}(\hat{\mathbf{x}})\right)} \sum_{i=1}^{l_{r}} \beta_{i} \hat{\xi}_{i}(\hat{\mathbf{x}})
$$

Coefficients $\left(\alpha_{1}, \cdots, \alpha_{l_{r}}\right)^{\top}$ and $\left(\beta_{1}, \cdots, \beta_{l_{r}}\right)^{\top}$ are obtained by solving the following two linear systems,

$$
A_{1}\left(\alpha_{1}, \cdots, \alpha_{l_{r}}\right)^{\top}=\mathbf{b}_{1} \quad \text { and } \quad A_{2}\left(\beta_{1}, \cdots, \beta_{l_{r}}\right)^{\top}=\mathbf{b}_{2}
$$

with

$$
\begin{aligned}
\left(A_{1}\right)_{i j}=\int_{T} \frac{\hat{\xi}_{i}(\hat{\mathbf{x}}(\mathbf{x})) \hat{\xi}_{j}(\hat{\mathbf{x}}(\mathbf{x})) d \mathbf{x}}{\operatorname{det}\left(D G_{T}(\hat{\mathbf{x}}(\mathbf{x}))\right)} \quad\left(\mathbf{b}_{1}\right)_{j}=\int_{T} u(\mathbf{x}) \hat{\xi}_{j}(\hat{\mathbf{x}}(\mathbf{x})) d \mathbf{x} \\
\left(A_{2}\right)_{i j}=\int_{T} \frac{\hat{\xi}_{i}(\hat{\mathbf{x}}(\mathbf{x})) \hat{\xi}_{j}(\hat{\mathbf{x}}(\mathbf{x})) d \mathbf{x}}{\left(\operatorname{det}\left(D G_{T}(\hat{\mathbf{x}}(\mathbf{x}))\right)\right)^{2}} \quad\left(\mathbf{b}_{2}\right)_{j}=\int_{T} \frac{\hat{\xi}_{j}(\hat{\mathbf{x}}(\mathbf{x})) d \mathbf{x}}{\operatorname{det}\left(D G_{T}(\hat{\mathbf{x}}(\mathbf{x}))\right)} u(\mathbf{x})
\end{aligned}
$$

for $1 \leq i, j \leq l_{r}$. By pulling back to $\hat{T}$ we obtain, for any $1 \leq i, j \leq l_{r}$,

$$
\begin{aligned}
&\left(A_{1}\right)_{i j}=\int_{\hat{T}} \hat{\xi}_{i}(\hat{\mathbf{x}}) \hat{\xi}_{j}(\hat{\mathbf{x}}) d \hat{\mathbf{x}}\left(\mathbf{b}_{1}\right)_{j}=\operatorname{det}\left(B_{T}\right) \int_{\hat{T}} \operatorname{det}\left(B_{T}^{-1} D G_{T}(\hat{\mathbf{x}})\right) u(\mathbf{x}) \hat{\xi}_{j}(\hat{\mathbf{x}}(\mathbf{x})) d \hat{\mathbf{x}} \\
&\left(A_{2}\right)_{i j}=\int_{\hat{T}} \frac{\hat{\xi}_{i}(\hat{\mathbf{x}}) \hat{\xi}_{j}(\hat{\mathbf{x}}) d \hat{\mathbf{x}}}{\operatorname{det}\left(D G_{T}(\hat{\mathbf{x}})\right)} \quad\left(\mathbf{b}_{2}\right)_{j}=\int_{\hat{T}} u(\mathbf{x}(\mathbf{x})) \hat{\xi}_{j}(\hat{\mathbf{x}}) d \hat{\mathbf{x}}
\end{aligned}
$$

Since $\operatorname{det}\left(B_{T}\right)$ is a non-zero constant, $\left(\operatorname{det}\left(B_{T}\right) A_{2}\right)\left(\beta_{1}, \cdots, \beta_{l_{r}}\right)^{\top}=\operatorname{det}\left(B_{T}\right) \mathbf{b}_{2}$. So we can redefine $A_{2}$ and $\mathbf{b}_{2}$ in the following way.

$$
\left(A_{2}\right)_{i j}=\int_{\hat{T}} \frac{\operatorname{det}\left(B_{T}\right)}{\operatorname{det}\left(D G_{T}(\hat{\mathbf{x}})\right)} \hat{\xi}_{i}(\hat{\mathbf{x}}) \hat{\xi}_{j}(\hat{\mathbf{x}}) d \hat{\mathbf{x}} \quad\left(\mathbf{b}_{2}\right)_{j}=\operatorname{det}\left(B_{T}\right) \int_{\hat{T}} u(\mathbf{x}(\hat{\mathbf{x}})) \hat{\xi}_{j}(\hat{\mathbf{x}}) d \hat{\mathbf{x}}
$$

According to Lemma 3.9, $\lim _{h \rightarrow 0} \sup _{T \in \mathcal{T}_{h}}\left\|A_{1}-A_{2}\right\|=0$. And, for any $\varepsilon>0$,

$$
\begin{aligned}
\lim _{h \rightarrow 0} \sup _{T \in \mathcal{T}_{h}}\left\|\mathbf{b}_{1}-\mathbf{b}_{2}\right\|^{2} & \leq\left(\varepsilon \operatorname{det}\left(B_{T}\right)\right)^{2} \int_{\hat{T}} u^{2}(\mathbf{x}(\hat{\mathbf{x}})) d \hat{\mathbf{x}} \\
& =\varepsilon^{2} \operatorname{det}\left(B_{T}\right) \int_{T} \operatorname{det}\left(B_{T} D G_{T}(\hat{\mathbf{x}}(\mathbf{x}))^{-1}\right) u^{2}(\mathbf{x}) d \mathbf{x} \\
& \leq 4 \varepsilon^{2} \operatorname{det}\left(B_{T}\right)\|u\|_{L^{2}(T)}^{2} .
\end{aligned}
$$

The last inequality holds when $h$ is small enough. This implies that

$$
\lim _{h \rightarrow 0} \sup _{T \in \mathcal{T}_{h}}\left\|\mathbf{b}_{1}-\mathbf{b}_{2}\right\| \leq 2 \varepsilon \sqrt{\operatorname{det}\left(B_{T}\right)}\|u\|_{L^{2}(T)} .
$$


So, for any $\varepsilon>0$, there exists $\delta>0$ such that, for any $h \leq \delta$ and $T \in \mathcal{T}_{h}$, we have

$$
\left\|\left(\alpha_{1}-\beta_{1}, \cdots, \alpha_{l_{r}}-\beta_{l_{r}}\right)\right\| \leq 3 \varepsilon \sqrt{\operatorname{det}\left(B_{T}\right)}\|u\|_{L^{2}(T)}
$$

We have then

$$
\begin{aligned}
\left\|\Pi_{\tilde{r}, T}^{2} u-P_{\tilde{r}, T} u\right\|_{L^{2}(T)}^{2} & =\int_{T} \frac{1}{\left(\operatorname{det}\left(D G_{T}(\hat{\mathbf{x}}(\mathbf{x}))\right)\right)^{2}} \sum_{i=1}^{l_{r}} \hat{\xi}_{i}^{2}(\hat{\mathbf{x}}(\mathbf{x}))\left(\alpha_{i}-\beta_{i}\right)^{2} d \mathbf{x} \\
& =\int_{\hat{T}} \frac{1}{\operatorname{det}\left(D G_{T}(\hat{\mathbf{x}})\right)} \sum_{i=1}^{l_{r}} \hat{\xi}_{i}^{2}(\hat{\mathbf{x}})\left(\alpha_{i}-\beta_{i}\right)^{2} d \hat{\mathbf{x}} \\
& \leq c \varepsilon^{2}\|u\|_{L^{2}(T)}^{2} \int_{\hat{T}} \operatorname{det}\left(B_{T} D G_{T}(\hat{\mathbf{x}})^{-1}\right) \sum_{i=1}^{l_{r}} \hat{\xi}_{i}^{2}(\hat{\mathbf{x}}) d \hat{\mathbf{x}}
\end{aligned}
$$

Here $c$ is a positive constant which depends on $l_{r}$ only. By Lemma 3.9, there exists $M>0$ such that, for any $h$ small enough and any $T \in \mathcal{T}_{h}$,

$$
\int_{\hat{T}} \operatorname{det}\left(B_{T} D G_{T}(\hat{\mathbf{x}})^{-1}\right) \sum_{i=1}^{l_{r}} \hat{\xi}_{i}^{2}(\hat{\mathbf{x}}) d \hat{\mathbf{x}} \leq M^{2}
$$

We can conclude that, for any $\varepsilon>0$, there exists $\delta>0$ such that, for any $h<\delta$ and $T \in \mathcal{T}_{h}$,

$$
\left\|\Pi_{\tilde{r}, T}^{2} u-P_{\tilde{r}, T} u\right\|_{L^{2}(T)} \leq \varepsilon\|u\|_{L^{2}(T)}, \forall u \in L^{2}(T)
$$

Here $P_{\tilde{r}, T}$ is the standard $L^{2}$-projection onto $\mathcal{P}_{\tilde{r}} \Lambda^{2}(T)$.

6.1. Projection Based Interpolation onto $\mathcal{P}_{\tilde{r}+1} \Lambda^{1}\left(\mathcal{T}_{h}\right)$.

Definition 6.6. For any $T \in \mathcal{T}_{h}$, we define a linear operator $\Pi_{\tilde{r}+1, T}^{1}: H^{1}\left(T ; \mathbb{R}^{2}\right) \longrightarrow$ $\mathcal{P}_{\tilde{r}+1} \Lambda^{1}(T)$ by the relations

$$
\begin{gathered}
\int_{T} \operatorname{div}\left(\Pi_{\tilde{r}+1, T}^{1} \omega-\omega\right)(\mathbf{x}) \hat{\psi}(\hat{\mathbf{x}}(\mathbf{x})) d \mathbf{x}=0 \quad \forall \hat{\psi} \in \mathcal{P}_{\tilde{r}(T)}(\hat{T}) / \mathbb{R} \\
\text { (6.4) } \int_{T}\left(\Pi_{\tilde{r}+1, T}^{1} \omega(\mathbf{x})-\omega(\mathbf{x})\right)^{\top} D G_{T}(\hat{\mathbf{x}})^{-\top} \operatorname{curl}_{\hat{\mathbf{x}}} \hat{\varphi}(\hat{\mathbf{x}}(\mathbf{x})) d \mathbf{x}=0 \quad \forall \hat{\varphi} \in \stackrel{\mathcal{P}}{\tilde{r}(T)+2}(\hat{T})
\end{gathered}
$$

$\int_{[0,1]}\left[\left(\Pi_{\tilde{r}+1, T}^{1} \omega-\omega\right)\left(\mathbf{x}_{e}(s)\right) \cdot \mathbf{n}\left(\mathbf{x}_{e}(s)\right)\right] \hat{\eta}(s)\left\|\dot{\mathbf{x}}_{e}(s)\right\| d s=0 \quad \forall \hat{\eta} \in \mathcal{P}_{\tilde{r}(e)+1}([0,1]) \forall e \in \triangle_{1}(T)$

Here $\mathbf{x}=G_{T}(\hat{\mathbf{x}})$ for any $\hat{\mathbf{x}} \in \hat{T}, \mathbf{x}_{e}(s):[0,1] \rightarrow e$ is the parameterization of $e$, and $\mathbf{n}$ is a unit normal vector along $e$ (the choice of its direction does not matter).

Definition 6.7. (Projection Based Interpolation operator onto $\mathcal{P}_{\tilde{r}+1} \Lambda^{1}(\hat{T})$ ) We define a linear operator $\Pi_{\tilde{r}+1, \hat{T}}^{1}: H(\hat{T}) \longrightarrow \mathcal{P}_{\tilde{r}+1} \Lambda^{1}(\hat{T})$ by the relations

$$
\begin{gathered}
\int_{\hat{T}} \operatorname{div}_{\hat{\mathbf{x}}}\left(\Pi_{\tilde{r}+1, \hat{T}}^{1} \hat{\omega}-\hat{\omega}\right) \hat{\psi} d \hat{\mathbf{x}}=0 \quad \forall \hat{\psi} \in \mathcal{P}_{\tilde{r}(\hat{T})}(\hat{T}) / \mathbb{R} \\
\int_{\hat{T}}\left(\Pi_{\tilde{r}+1, \hat{T}}^{1} \hat{\omega}-\hat{\omega}\right) \cdot \operatorname{curl}_{\hat{\mathbf{x}}} \hat{\varphi} d \hat{\mathbf{x}}=0 \quad \forall \hat{\varphi} \in \stackrel{\mathcal{P}}{\tilde{r}(\hat{T})+2}(\hat{T}) \\
\int_{\hat{e}}\left(\Pi_{\tilde{r}+1, \hat{T}}^{1} \hat{\omega}-\hat{\omega}\right) \cdot \hat{\mathbf{n}} \hat{\eta} d \hat{s}=0 \quad \forall \hat{\eta} \in \mathcal{P}_{\tilde{r}(\hat{e})+1}(\hat{e}) \forall \hat{e} \in \triangle_{1}(\hat{T})
\end{gathered}
$$


Remark 6.8. The operator $\Pi_{\tilde{r}+1, \hat{T}}^{1}$ is the Projection Based Interpolation operator onto $\mathcal{P}_{\tilde{r}+1} \Lambda^{1}(\hat{T})$ defined in [19]. The operator $\Pi_{\tilde{r}+1, T}^{1}$ is defined by the pull back mapping from $\hat{T}$ to $T$.

Lemma 6.9. For any $T \in \mathcal{T}_{h}$, any $\omega \in\left[H^{1}(T)\right]^{2}$, we define $\hat{\omega}$ by the relation

$$
\omega(\mathbf{x}(\hat{\mathbf{x}}))=\frac{D G_{T}(\hat{\mathbf{x}})}{\operatorname{det}\left(D G_{T}(\hat{\mathbf{x}})\right)} \hat{\omega}(\hat{x})
$$

Then $\hat{\omega}(\hat{\mathbf{x}}) \in H(\hat{T})$, and

$$
\Pi_{\tilde{r}+1, T}^{1} \omega(\mathbf{x}(\hat{\mathbf{x}}))=\frac{D G_{T}(\hat{\mathbf{x}})}{\operatorname{det}\left(D G_{T}(\hat{\mathbf{x}})\right)} \Pi_{\tilde{r}+1, \hat{T}}^{1} \hat{\omega}(\hat{\mathbf{x}})
$$

Proof. Obviously, $\hat{\omega}(\hat{\mathbf{x}})=\operatorname{det}\left(D G_{T}(\hat{\mathbf{x}})\right)\left(D G_{T}(\hat{\mathbf{x}})\right)^{-1} \omega\left(G_{T}(\hat{\mathbf{x}})\right)$. Using Lemma 3.12, we can conclude that $\hat{\omega}(\hat{\mathbf{x}}) \in H(\hat{T})$.

By pulling back to $\hat{T}$ and using the definition of $\mathcal{P}_{\tilde{r}+1} \Lambda^{1}(T)$, we can see that $(6.4)$ is the same as (6.7), and (6.3) is the same as (6.6). Thus, we only need to show that (6.5) is the same as (6.8). Since $\mathcal{T}_{h}$ is $C^{0}$-compatible, then $G_{T}(\zeta(s))=\mathbf{x}_{e}(s)$ for any $s \in[0,1]$ where $\zeta:[0,1] \rightarrow \hat{e}$ is an affine local parameterization of $\hat{e}$.

We have then

$$
\begin{aligned}
& \int_{[0,1]}\left[\omega\left(\mathbf{x}_{e}(s)\right) \cdot \mathbf{n}\left(\mathbf{x}_{e}(s)\right)\right] \hat{\eta}(s)\left\|\dot{\mathbf{x}}_{e}(s)\right\| d s \\
= & \int_{[0,1]}\left[\omega\left(G_{T}(\zeta(s))\right) \cdot \mathbf{n}\left(G_{T}(\zeta(s))\right)\right] \hat{\eta}(s)\left\|D G_{T}(\zeta(s)) \dot{\zeta}(s)\right\| d s \\
= & \int_{[0,1]}\left[\frac{D G_{T}(\zeta(s)) \hat{\omega}(\zeta(s))}{\operatorname{det}\left(D G_{T}(\zeta(s))\right)} \cdot \frac{\left(D G_{T}(\zeta(s))\right)^{-\top} \hat{\mathbf{n}}(\zeta(s))}{\left\|\left(D G_{T}(\zeta(s))\right)^{-\top} \hat{\mathbf{n}}(\zeta(s))\right\|}\right] \hat{\eta}(s)\left\|D G_{T}(\zeta(s)) \dot{\zeta}(s)\right\| d s \\
= & \int_{[0,1]}\left[\frac{\hat{\omega}(\zeta(s))}{\operatorname{det}\left(D G_{T}(\zeta(s))\right)} \cdot \frac{\hat{\mathbf{n}}(\zeta(s))}{\left\|\left(D G_{T}(\zeta(s))\right)^{-\top} \hat{\mathbf{n}}(\zeta(s))\right\|}\right] \hat{\eta}(s)\left\|D G_{T}(\zeta(s)) \dot{\zeta}(s)\right\| d s
\end{aligned}
$$

Notice that $\zeta(s)=c \hat{t}$ where $\hat{t}$ is a unit tangent vector along $\hat{e}$, and $c$ is a nonzero constant, $\hat{\mathbf{n}}(\zeta(s))=\left(\hat{t}_{2},-\hat{t}_{1}\right)^{\top}$, and $\left(D G_{T}(\zeta(s))\right)^{-\top}=\frac{A}{\operatorname{det}\left(D G_{T}(\zeta(s))\right)}$ with

$$
A=\left[\begin{array}{ll}
\left(D G_{T}\right)_{22}(\zeta(s)) & -\left(D G_{T}\right)_{21}(\zeta(s)) \\
-\left(D G_{T}\right)_{12}(\zeta(s)) & \left(D G_{T}\right)_{11}(\zeta(s))
\end{array}\right]
$$

Therefore, we have

$$
\begin{aligned}
& \int_{[0,1]}\left[\frac{\hat{\omega}(\zeta(s))}{\operatorname{det}\left(D G_{T}(\zeta(s))\right)} \cdot \frac{\hat{\mathbf{n}}(\zeta(s))}{\left\|\left(D G_{T}(\zeta(s))\right)^{-\top} \hat{\mathbf{n}}(\zeta(s))\right\|}\right] \hat{\eta}(s)\left\|D G_{T}(\zeta(s)) \dot{\zeta}(s)\right\| d s \\
= & c \int_{[0,1]}[\hat{\omega}(\zeta(s)) \cdot \hat{\mathbf{n}}(\zeta(s))] \hat{\eta}(s) d s
\end{aligned}
$$

We conclude that (6.5) is equivalent with (6.8). This finishes the proof.

Lemma 6.10. For any $T \in \mathcal{T}_{h}$, and any $\omega \in\left[H^{1}(T)\right]^{2}$, we have $\Pi_{\tilde{r}, T}^{2}$ divw = $\operatorname{div} \prod_{\tilde{r}+1, T}^{1} \omega$.

Proof. By Lemma 3.12, we have $\hat{\omega}(\hat{\mathbf{x}}) \in H(\hat{T})$, and

$$
\operatorname{div} \omega(\mathbf{x}(\hat{\mathbf{x}}))=\frac{1}{\operatorname{det}\left(D G_{T}(\hat{\mathbf{x}})\right)} \operatorname{div}_{\hat{\mathbf{x}}} \hat{\omega}(\hat{\mathbf{x}})
$$


for $\hat{\mathbf{x}} \in \hat{T}$ almost everywhere, provided we define $\hat{\omega}(\hat{\mathbf{x}})$ on $\hat{T}$ by

$$
\omega(\mathbf{x}(\hat{\mathbf{x}}))=\frac{D G_{T}(\hat{\mathbf{x}})}{\operatorname{det}\left(D G_{T}(\hat{\mathbf{x}})\right)} \hat{\omega}(\hat{\mathbf{x}})
$$

for any $\hat{\mathbf{x}} \in \hat{T}$.

Using Definition 6.1, Definition 6.2, Lemma 6.4, Lemma 6.9, and Lemma 10 in [19], it is easy to see that

$$
\begin{aligned}
& \Pi_{\tilde{r}, T}^{2} \operatorname{div} \omega(\mathbf{x}(\hat{\mathbf{x}}))=\frac{1}{\operatorname{det}\left(D G_{T}(\hat{\mathbf{x}})\right)} \Pi_{\tilde{r}, \hat{T}}^{2} \operatorname{div}_{\hat{\mathbf{x}}} \hat{\omega}(\hat{\mathbf{x}})=\frac{1}{\operatorname{det}\left(D G_{T}(\hat{\mathbf{x}})\right)} \operatorname{div}_{\hat{\mathbf{x}}} \Pi_{\tilde{r}+1, \hat{T}}^{1} \hat{\omega}(\hat{\mathbf{x}}) \\
& \operatorname{div} \Pi_{\tilde{r}+1, T}^{1} \omega(\mathbf{x}(\hat{\mathbf{x}}))=\operatorname{div}\left[\frac{D G_{T}(\hat{\mathbf{x}})}{\operatorname{det}\left(D G_{T}(\hat{\mathbf{x}})\right)} \Pi_{\tilde{r}+1, \hat{T}}^{1} \hat{\omega}(\hat{\mathbf{x}})\right]=\frac{1}{\operatorname{det}\left(D G_{T}(\hat{\mathbf{x}})\right)} \operatorname{div}_{\hat{\mathbf{x}}} \Pi_{\tilde{r}+1, \hat{T}}^{1} \hat{\omega}(\hat{\mathbf{x}})
\end{aligned}
$$

We have thus $\Pi_{\tilde{r}, T}^{2} \operatorname{div} \omega=\operatorname{div} \Pi_{\tilde{r}+1, T}^{1} \omega$.

Lemma 6.11. There exists $\delta>0$ and $C>0$ such that, for any $h<\delta$, we have

$$
\left\|\Pi_{\tilde{r}, T}^{1} \omega\right\|_{L^{2}(T)} \leq C\|\omega\|_{H^{1}(T)} \quad \forall T \in \mathcal{T}_{h}, \omega \in H^{1}\left(T ; \mathbb{R}^{2}\right)
$$

For affine meshes, the inequality above holds for any $h>0$.

Proof. According to Lemma 3.13 and Lemma 3.14, there exist $\delta>0$ and $C_{1}>0$ such that, for any $h<\delta$ and $T \in \mathcal{T}_{h}$,

$$
\begin{gathered}
\left\|\Pi_{\tilde{r}, T}^{1} \omega\right\|_{L^{2}(T)} \leq C_{1}\left\|\Pi_{\tilde{r}, \hat{T}}^{1} \hat{\omega}\right\|_{L^{2}(\hat{T})} \quad \forall \omega \in L^{2}\left(T ; \mathbb{R}^{2}\right) \\
\|\hat{\omega}\|_{H\left(\operatorname{div}_{\tilde{x}}, T\right)}^{2}+\|\hat{\omega}\|_{L^{2}(\partial \hat{T})}^{2} \leq C_{1}\|\omega\|_{H^{1}(T)} \quad \forall \omega \in H^{1}\left(T ; \mathbb{R}^{2}\right)
\end{gathered}
$$

By Lemma $6.9, \hat{\omega} \in H^{1}\left(\hat{T} ; \mathbb{R}^{2}\right)$ for any $\omega \in H^{1}\left(T ; \mathbb{R}^{2}\right)$.

The definition of operator $\Pi_{\tilde{r}, \hat{T}}$ implies that there exists a constant $C_{2}>0$ such that

$$
\int_{\hat{T}}\left(\Pi_{\tilde{r}, \hat{T}}^{1} \hat{\omega}(\hat{\mathbf{x}})\right)^{\top} \Pi_{\tilde{r}, \hat{T}}^{1} \hat{\omega}(\hat{\mathbf{x}}) d \hat{\mathbf{x}} \leq C_{2}\left(\|\hat{\omega}\|_{H\left(\operatorname{div}_{\hat{\mathbf{x}}}, \hat{T}\right)}^{2}+\|\hat{\omega}\|_{L^{2}(\partial(\hat{T}))}^{2}\right), \forall \hat{\omega}(\hat{\mathbf{x}}) \in\left[H^{1}(\hat{T})\right]^{2} .
$$

It is easy to see that for affine meshes the above inequality holds for any $h>0$. This finishes the proof.

6.2. Modified Projection Based Interpolation onto $\mathcal{P}_{r \tilde{+} 1}^{-} \Lambda^{1}(T)$ and modified operator $W$ onto $\mathcal{P}_{r \tilde{+} 2} \Lambda^{0}(T)$. In order to prove the stability of the mixed FE method, we need to make the left and right diagrams in (5.5) commute. In [19], the definition of Projection Based (PB) interpolation operator onto $\mathcal{P}_{\tilde{r}+1}^{-} \Lambda^{1}(\hat{T})$ was very similar to $\mathcal{P}_{\tilde{r}+1} \Lambda^{1}(\hat{T})$. From the proof of Lemma 10 in [19], we can see that only conditions (6.6) and (6.8) are used to prove the commutativity of the middle diagram in (5.5). This implies that we may be able to change condition (6.7) for the PB interpolation operator onto $\mathcal{P}_{\tilde{r}+1}^{-} \Lambda^{1}(\hat{T})$ in such a way that we can design a new operator $W_{h}$ which makes the right diagram in (5.5) commute, and can be proved to be well-defined, for an arbitrary order of discretization.

Definition 6.12. Let $\tilde{r}: \triangle(\hat{T}) \rightarrow \mathbb{Z}_{+}$be a mapping that prescribes the local order of discretization and satisfies the minimum rule, i.e. if $\hat{e}, \hat{f} \in \triangle(\hat{T})$ and $\hat{e} \subset \hat{f}$ then $\tilde{r}(\hat{e}) \leq \tilde{r}(\hat{f})$. We put $k_{\tilde{r}}=\operatorname{dim} \operatorname{curl}_{\hat{\mathbf{x}}} \stackrel{\circ}{\mathcal{P}}_{\tilde{r}(\hat{T})+1}(\hat{T})$. Let $\left\{\hat{\mathbf{f}}_{\tilde{r}, 1}(\hat{\mathbf{x}}), \cdots, \hat{\mathbf{f}}_{\tilde{r}, k_{\tilde{r}}}(\hat{\mathbf{x}})\right\}$ be 
a basis of $\operatorname{curl}_{\hat{\mathbf{x}}} \stackrel{\circ}{\tilde{r}(\hat{T})+1}(\hat{T})$. Let $\left\{\hat{\mathbf{g}}_{\tilde{r}, 1}(\hat{\mathbf{x}}), \cdots, \hat{\mathbf{g}}_{\tilde{r}, k_{\tilde{r}}}(\hat{\mathbf{x}})\right\}$ be a linearly independent subset of $\mathcal{P}_{\tilde{r}(\hat{T})-1}\left(\hat{T} ; \mathbb{R}^{2}\right)$ such that

$$
\operatorname{span}\left\{\hat{\mathbf{g}}_{\tilde{r}, 1}(\hat{\mathbf{x}}), \cdots, \hat{\mathbf{g}}_{\tilde{r}, k_{\tilde{r}}}(\hat{\mathbf{x}})\right\} \oplus \operatorname{grad}_{\hat{\mathbf{x}}} \mathcal{P}_{\tilde{r}(\hat{T})}(\hat{T})=\left[\mathcal{P}_{\tilde{r}(\hat{T})-1}(\hat{T})\right]^{2}
$$

For $t \in[0,1]$, we define $\hat{\mathbf{h}}_{\tilde{r}, i}(\hat{\mathbf{x}}, t)=(1-t) \hat{\mathbf{f}}_{\tilde{r}, 1}(\hat{\mathbf{x}})+t \hat{\mathbf{g}}_{\tilde{r}, 1}(\hat{\mathbf{x}}), 1 \leq i \leq k_{\tilde{r}}$.

Remark 6.13. It is easy to check that $k_{\tilde{r}}=\operatorname{dim} \mathcal{P}_{\tilde{r}(\hat{T})-1}\left(\hat{T} ; \mathbb{R}^{2}\right)-\operatorname{dim} \operatorname{grad}_{\hat{\mathbf{x}}} \mathcal{P}_{\tilde{r}(\hat{T})}(\hat{T})$.

Definition 6.14. (One-parameter family of PB interpolation operators onto $\mathcal{P}_{\tilde{r}+1}^{-} \Lambda^{1}(T)$ ) For $t \in[0,1]$, and for any $T \in \mathcal{T}_{h}$, we define a linear operator $\Pi_{\tilde{r}+1, T, t}^{1,-}: H^{1}\left(T ; \mathbb{R}^{2}\right) \longrightarrow$ $\mathcal{P}_{\tilde{r}+1}^{-} \Lambda^{1}(T)$ by the relations

$$
\int_{T} \operatorname{div}\left(\Pi_{\tilde{r}+1, T, t}^{1,-} \omega-\omega\right)(\mathbf{x}) \hat{\psi}(\hat{\mathbf{x}}(\mathbf{x})) d \mathbf{x}=0 \quad \forall \hat{\psi} \in \mathcal{P}_{\tilde{r}(T)}(\hat{T}) / \mathbb{R}
$$

$\int_{[0,1]}^{0}\left[\left(\Pi_{\tilde{r}+1, T, t}^{1,-} \omega-\omega\right)\left(\mathbf{x}_{e}(s)\right) \cdot \mathbf{n}\left(\mathbf{x}_{e}(s)\right)\right] \hat{\eta}(s)\left\|\dot{\mathbf{x}}_{e}(s)\right\| d s=0 \quad \forall \hat{\eta} \in \mathcal{P}_{\tilde{r}(e)}([0,1]), \forall e \in \triangle_{1}(T)$

$$
\int_{T}\left(\Pi_{\tilde{r}+1, T, t}^{1,-} \omega(\mathbf{x})-\omega(\mathbf{x})\right)^{\top} D G_{T}(\hat{\mathbf{x}}(\mathbf{x}))^{-\top} \hat{\mathbf{h}}_{i}(\hat{\mathbf{x}}(\mathbf{x}), t) d \mathbf{x}=0 \quad 1 \leq i \leq k_{\tilde{r}}
$$

In the above, $\hat{\mathbf{x}}=\hat{\mathbf{x}}(\mathbf{x})$ signifies the inverse of the element map.

Definition 6.15. (One-parameter family of PB interpolation operators onto $\mathcal{P}_{\tilde{r}+1}^{-} \Lambda^{1}(\hat{T})$ ) For any $t \in[0,1]$, we define a linear operator $\Pi_{\tilde{r}+1, \hat{T}, t}^{1,-}: H(\hat{T}) \longrightarrow \mathcal{P}_{\tilde{r}+1}^{-} \Lambda^{1}(\hat{T})$ by the relations

$$
\begin{gathered}
\int_{\hat{T}} \operatorname{div}_{\hat{\mathbf{x}}}\left(\Pi_{\tilde{r}+1, \hat{T}, t}^{1,-} \hat{\omega}-\hat{\omega}\right)(\hat{\mathbf{x}}) \hat{\psi}(\hat{\mathbf{x}}) d \hat{\mathbf{x}}=0 \quad \forall \hat{\psi} \in \mathcal{P}_{\tilde{r}(T)}(\hat{T}) / \mathbb{R} \\
\int_{\hat{T}}\left(\Pi_{\hat{r}+1, \hat{T}, t}^{1,-} \hat{\omega}(\hat{\mathbf{x}})-\hat{\omega}(\hat{\mathbf{x}})\right)^{\top} \hat{\mathbf{h}}_{i}(\hat{\mathbf{x}}, t) d \hat{\mathbf{x}}=0 \quad 1 \leq i \leq k_{\tilde{r}} \\
\int_{\hat{e}}\left[\left(\Pi_{\tilde{r}+1, \hat{T}, t}^{1,-} \hat{\omega}-\hat{\omega}\right) \cdot \hat{\mathbf{n}}\right] \hat{\eta} d \hat{s}=0 \quad \forall \hat{\eta} \in \mathcal{P}_{\tilde{r}(\hat{e})}(\hat{e}), \forall \hat{e} \in \triangle_{1}(\hat{T})
\end{gathered}
$$

Definition 6.16. In the sequel, the phrase "for almost all" (parameters) will mean "for all except for a finite number" (of parameters). For example, a sequence $x_{n}$ in a topological space converges to $x$ if, for every neighborhood of $x$, almost all values of $x_{n}$ belong to the neighborhood.

Lemma 6.17. $\Pi_{\tilde{r}+1, \hat{T}, t}^{1,-}: H(\hat{T}) \longrightarrow \mathcal{P}_{\tilde{r}+1}^{-} \Lambda^{1}(\hat{T})$ is a well-defined linear operator for almost all $t \in[0,1]$. Moreover,

$$
\operatorname{div}_{\hat{\mathbf{x}}} \Pi_{\tilde{r}+1, \hat{T}, t}^{1,-} \hat{\omega}=\Pi_{\tilde{r}, \hat{T}}^{2} d i v_{\hat{\mathbf{x}}} \hat{\omega}
$$

for any $\hat{\omega} \in H(\hat{T})$.

Proof. The linearity of the operator is obvious, for any $t \in \mathbb{R}$. The point is to show that the operator is well-defined. For $t=0$, the operator $\Pi_{\tilde{r}+1, \hat{T}, 0}^{1,-}$ reduces to the $\mathrm{PB}$ interpolation defined in [19] and, according to Lemma 9 and Lemma 20 from [19], is well-defined. Moreover,

- $\int_{\hat{T}} \operatorname{div}_{\hat{\mathbf{x}}} \hat{\omega}(\hat{\mathbf{x}}) \hat{\psi}(\hat{\mathbf{x}}) d \hat{x}_{1} d \hat{x}_{2}$ is a continuous functional of $\hat{\omega}$, for any $\hat{\psi} \in \mathcal{P}_{\tilde{r}(T)}(\hat{T}) / \mathbb{R}$, 
- $\int_{\hat{T}} \hat{\omega}(\hat{\mathbf{x}})^{\top} \hat{\mathbf{h}}_{i}(\hat{\mathbf{x}}, t) d \hat{x}_{1} d \hat{x}_{2}$ is a continues functional of $\hat{\omega}$, for any $1 \leq i \leq k_{\tilde{r}}$ and any $t \in \mathbb{R}$, and

- $\int_{\hat{e}}[\hat{\omega} \cdot \hat{\mathbf{n}}] \hat{\eta} d \hat{s}$ is a continues functional of $\hat{\omega}$, for any $\hat{\eta} \in \mathcal{P}_{\tilde{r}(\hat{e})}(\hat{e})$, and any $\hat{e} \in \triangle_{1}(\hat{T})$.

Therefore, in order to demonstrate that $\Pi_{\tilde{r}+1, \hat{T}, t}^{1,-}$ is well-defined, it is sufficient to show that $\hat{\omega}=0$ if $\hat{\omega} \in \mathcal{P}_{\tilde{r}+1}^{-} \Lambda^{1}(\hat{T})$ and $\Pi_{\tilde{r}+1, \hat{T}, t}^{1,-} \hat{\omega}=0$.

We take an arbitrary $\hat{\omega} \in \mathcal{P}_{\tilde{r}+1}^{-} \Lambda^{1}(\hat{T})$ such that $\Pi_{\tilde{r}+1, \hat{T}, t}^{1,-} \hat{\omega}=0$. According to the definition of $\mathcal{P}_{\tilde{r}+1}^{-} \Lambda^{1}(\hat{T})$ and $(6.14)$, we know that $\hat{\omega} \in \stackrel{\circ}{\mathcal{P}}_{\tilde{r}+1}^{-} \Lambda^{1}(\hat{T})$. Set $r=\tilde{r}(\hat{T})$. We denote by $C(t, r)$ the matrix associated with conditions (6.12) and (6.13), corresponding to a particular basis for $\stackrel{\mathcal{P}}{\tilde{r}+1}^{-} \Lambda^{1}(\hat{T})$ (the solution space), and a particular basis for $\mathcal{P}_{\tilde{r}(T)}(\hat{T}) / \mathbb{R}$. We argue that matrix $C(t, r)$ is non-singular for almost all $t \in[0,1]$. Notice that, for any $r$, $\operatorname{det}(C(t, r))$ is a polynomial in $t$. Since $\Pi_{\tilde{r}+1, \hat{T}, 0}^{1,-}$ is well-defined, $\operatorname{det}(C(0, r)) \neq 0$. So $\operatorname{det}(C(t, r))$ is a non-zero polynomial. By the fundamental theorem of algebra, the polynomial has a finite number of real roots. We can conclude thus that $\Pi_{\tilde{r}+1, \hat{T}, t}^{1,-}: H(\hat{T}) \longrightarrow \mathcal{P}_{\tilde{r}+1}^{-} \Lambda^{1}(\hat{T})$ is well-defined for any $t \in[0,1]$ except for the roots of $\operatorname{det}(C(t, r))$. The number of roots is independent of the choice of the bases and depends only upon $r=\tilde{r}(\hat{T})$.

Since $\operatorname{div}_{\hat{\mathbf{x}}} \Pi_{\tilde{r}+1, \hat{T}, t}^{1,-} \hat{\omega} \in \mathcal{P}_{\tilde{r}} \Lambda^{2}(\hat{T})$, for any $\hat{\omega} \in H(\hat{T})$, then $\Pi_{\tilde{r}, \hat{T}}^{2} \operatorname{div}_{\hat{\mathbf{x}}} \Pi_{\tilde{r}+1, \hat{T}, t}^{1,-} \hat{\omega}=$ $\operatorname{div}_{\hat{\mathbf{x}}} \Pi_{\tilde{r}+1, \hat{T}, t}^{1,-} \hat{\omega}$. In order to show that $\operatorname{div}_{\hat{\mathbf{x}}} \Pi_{\tilde{r}+1, \hat{T}, t}^{1,-} \hat{\omega}=\Pi_{\tilde{r}, \hat{T}}^{2} \operatorname{div}_{\hat{\mathbf{x}}} \hat{\omega}$, it is sufficient to show that $\Pi_{\tilde{r}, \hat{T}}^{2} \operatorname{div}_{\hat{\mathbf{x}}} \hat{\omega}=0$, for any $\hat{\omega}$ such that $\Pi_{\tilde{r}+1, \hat{T}, t}^{1,-} \hat{\omega}=0$. We have

$$
\int_{\hat{T}} \operatorname{div}_{\hat{\mathbf{x}}} \hat{\omega} \hat{\psi} d \hat{\mathbf{x}}=\int_{\hat{T}} \operatorname{div}_{\hat{\mathbf{x}}} \hat{\omega}(\hat{\chi}+c) d \hat{\mathbf{x}}=\int_{\hat{T}} \operatorname{div}_{\hat{\mathbf{x}}} \hat{\omega} \hat{\chi} d \hat{\mathbf{x}}+c \int_{\partial \hat{T}} \hat{\omega} \cdot \hat{\mathbf{n}} d \hat{s}=0 .
$$

Here $\Pi_{\tilde{r}+1, \hat{T}, t}^{1,-} \hat{\omega}=0, \hat{\psi} \in \mathcal{P}_{\tilde{r}(\hat{T})}(\hat{T}), c=\int_{\hat{T}} \hat{\psi} d \hat{\mathbf{x}}$. The last equality holds due to the definition of $\Pi_{\tilde{r}+1, \hat{T}, t}^{1,-}$ and the fact that $\Pi_{\tilde{r}+1, \hat{T}, t}^{1,-} \hat{\omega}=0$. We have thus $\operatorname{div}_{\hat{\mathbf{x}}} \Pi_{\tilde{r}+1, \hat{T}, t}^{1,-} \hat{\omega}=\Pi_{\tilde{r}, \hat{T}}^{2} \operatorname{div}_{\hat{\mathbf{x}}} \hat{\omega}$ for any $\hat{\omega} \in H^{1}\left(\hat{T} ; \mathbb{R}^{2}\right)$.

Theorem 6.18. Let $t \in[0,1]$ be any value for which $\Pi_{\tilde{r}+1, \hat{T}, t}^{1,-}$ is well-defined. Then the operator $\Pi_{\tilde{r}+1, T, t}^{1,-}: H^{1}\left(T ; \mathbb{R}^{2}\right) \longrightarrow \mathcal{P}_{\tilde{r}+1}^{-} \Lambda^{1}(T)$ is well-defined as well, and we have the following result.

There exist $\delta>0$ and $C>0$ such that, for $h \leq \delta, T \in \mathcal{T}_{h}$, and $\omega \in H^{1}\left(T ; \mathbb{R}^{2}\right)$, the corresponding function $\hat{\omega}$ defined by

$$
\omega(\mathbf{x}(\hat{\mathbf{x}}))=\frac{D G_{T}(\hat{\mathbf{x}})}{\operatorname{det}\left(D G_{T}(\hat{\mathbf{x}})\right)} \hat{\omega}(\hat{x})
$$

belongs to $H(\hat{T})$, and

Additionally,

$$
\Pi_{\tilde{r}+1, T, t}^{1,-} \omega(\mathbf{x}(\hat{\mathbf{x}}))=\frac{D G_{T}(\hat{\mathbf{x}})}{\operatorname{det}\left(D G_{T}(\hat{\mathbf{x}})\right)} \Pi_{\tilde{r}+1, \hat{T}, t}^{1,-} \hat{\omega}(\hat{\mathbf{x}})
$$

and

$$
\operatorname{div} \prod_{\tilde{r}+1, T, t}^{1,-} \omega=\Pi_{\tilde{r}, T}^{2} \operatorname{div} \omega
$$

$$
\left\|\Pi_{\tilde{r}+1, T, t}^{1,-} \omega\right\|_{L^{2}(T)} \leq C\|\omega\|_{H^{1}(T)}
$$


Proof. The proof is analogous to that of Lemma 6.9, Lemma 6.10, and Lemma 6.11.

Definition 6.19. For any $T \in \mathcal{T}_{h}$, we define a linear operator $C_{T, t}:\left[H^{1}(\hat{T})\right]^{2} \longrightarrow$ $\mathcal{P}_{\tilde{r}+2} \Lambda^{0}\left(\hat{T} ; \mathbb{R}^{2}\right)$ by the following relations

$$
\begin{array}{r}
\int_{\hat{T}} \operatorname{div}_{\hat{\mathbf{x}}} C_{T, t} \hat{\omega}(\hat{\mathbf{x}}) \hat{\psi}(\hat{\mathbf{x}}) d \hat{\mathbf{x}}=\int_{\hat{T}} \operatorname{div}_{\hat{\mathbf{x}}} \hat{\omega}(\hat{\mathbf{x}}) \hat{\psi}(\hat{\mathbf{x}}) d \hat{\mathbf{x}} \quad \forall \hat{\psi} \in \mathcal{P}_{\tilde{r}(\hat{T})}(\hat{T}) / \mathbb{R} \\
\int_{\hat{T}}\left(C_{T, t} \hat{\omega}(\hat{\mathbf{x}})\right)^{\top} \hat{\mathbf{h}}_{i}(\hat{\mathbf{x}}, t) d \hat{\mathbf{x}}=\int_{\hat{T}}(\hat{\omega}(\hat{\mathbf{x}}))^{\top} \hat{\mathbf{h}}_{i}(\hat{\mathbf{x}}, t) d \hat{\mathbf{x}} \quad 1 \leq i \leq k_{\tilde{r}} \\
\int_{[0,1]}\left[\left(C_{T, t} \hat{\omega}\right)(\zeta(s)) \cdot \hat{\mathbf{n}}(\zeta(s))\right] \hat{\eta}(s) d s=\int_{[0,1]}[(\hat{\omega})(\zeta(s)) \cdot \hat{\mathbf{n}}(\zeta(s))] \hat{\eta}(s) d s \\
\forall \hat{\eta} \in \mathcal{P}_{\tilde{r}(e)}([0,1]), \forall \hat{e} \in \triangle_{1}(\hat{T}) \\
\int_{[0,1]}\left[\left(C_{T, t} \hat{\omega}\right)(\zeta(s)) \cdot \hat{\mathbf{t}}(\zeta(s))\right] \hat{\eta}(s) d s= \\
\int_{[0,1]}[(\hat{\omega})(\zeta(s)) \cdot \hat{\mathbf{t}}(\zeta(s))] \hat{\eta}(s) d s \\
\forall \hat{\eta} \in \mathcal{P}_{\tilde{r}(e)}([0,1]), \forall \hat{e} \in \triangle_{1}(\hat{T})
\end{array}
$$

Here $\zeta(s):[0,1] \rightarrow \hat{e}$ is the affine local parameterization of $\hat{e}$.

Lemma 6.20. Operator $C_{T, t}: H^{1}\left(\hat{T} ; \mathbb{R}^{2}\right) \longrightarrow \mathcal{P}_{\tilde{r}+2} \Lambda^{0}\left(\hat{T} ; \mathbb{R}^{2}\right)$ is well-defined for almost all $t \in[0,1]$.

Proof. Take an arbitrary $t \in[0,1]$. Since $\hat{\omega} \in H^{1}\left(\hat{T} ; \mathbb{R}^{2}\right)$, then all right hand sides of $(6.15),(6.16),(6.17),(6.18)$ are continuous functionals with respect to $\hat{\omega}$. In order to show the well-definedness of $C_{T, t}$, it is sufficient to show that $\hat{\omega}=0$ if $\hat{\omega} \in \mathcal{P}_{\tilde{r}+2} \Lambda^{0}\left(\hat{T} ; \mathbb{R}^{2}\right), \hat{\omega}=0$ at all vertices of $\hat{T}$, and $C_{T, t} \hat{\omega}=0$. According to conditions $(6.17)$ and $(6.18), \hat{\omega} \in \dot{\mathcal{P}}_{\tilde{r}+2} \Lambda^{0}\left(\hat{T} ; \mathbb{R}^{2}\right)$. Define now $\dot{C}_{T, t}: H^{1}\left(\hat{T} ; \mathbb{R}^{2}\right) \longrightarrow$ $\stackrel{\circ}{\mathcal{P}}_{\tilde{r}+2} \Lambda^{0}\left(\hat{T} ; \mathbb{R}^{2}\right)$ by the relations

$$
\begin{gathered}
\int_{\hat{T}} \operatorname{div}_{\hat{\mathbf{x}}} \stackrel{\circ}{C}_{T, t} \hat{\omega}(\hat{\mathbf{x}}) \hat{\psi}(\hat{\mathbf{x}}) d \hat{\mathbf{x}}=\int_{\hat{T}} \operatorname{div}_{\hat{\mathbf{x}}} \hat{\omega}(\hat{\mathbf{x}}) \hat{\psi}(\hat{\mathbf{x}}) d \hat{\mathbf{x}} \quad \forall \hat{\psi}(\hat{\mathbf{x}}) \in \mathcal{P}_{\tilde{r}(\hat{T})}(\hat{T}) / \mathbb{R} \\
\int_{\hat{T}}\left(\stackrel{\circ}{C}_{T, t} \hat{\omega}(\hat{\mathbf{x}})\right)^{\top} \hat{\mathbf{h}}_{i}(\hat{\mathbf{x}}, t) d \hat{\mathbf{x}}=\int_{\hat{T}}(\hat{\omega}(\hat{\mathbf{x}}))^{\top} \hat{\mathbf{h}}_{i}(\hat{\mathbf{x}}, t) d \hat{\mathbf{x}} \quad 1 \leq i \leq k_{\tilde{r}}
\end{gathered}
$$

It is sufficient to show that operator $\stackrel{\circ}{C}_{T, t}: H^{1}\left(\hat{T} ; \mathbb{R}^{2}\right) \longrightarrow \stackrel{\circ}{\mathcal{P}}_{\tilde{r}+2} \Lambda^{0}\left(\hat{T} ; \mathbb{R}^{2}\right)$ is welldefined.

Obviously, the right-hand side of conditions (6.20) and (6.21) are continuous functionals with respect to $\hat{\omega}$. Set $r=\tilde{r}(\hat{T})$ and denote by $\stackrel{\circ}{C}(t, r)$ the matrix associated with the left-hand side of conditions (6.20) and (6.21) corresponding to some basis of ${\stackrel{\circ}{\mathcal{P}}+{ }_{\tilde{r}}}^{-} \Lambda^{0}\left(\hat{T} ; \mathbb{R}^{2}\right)$ (the solution space), and some basis of $\mathcal{P}_{\tilde{r}(T)}(\hat{T}) / \mathbb{R}$. We need to show that the $\operatorname{det}(\stackrel{\circ}{C}(t, r)) \neq 0$ for almost all $t \in[0,1]$.

Since $\hat{\omega}$ vanishes on the boundary, we can integrate by parts (6.20) without getting any boundary terms. So (6.20) is the same as (6.22)

$$
\int_{\hat{T}}\left(\stackrel{C}{C}_{T, t}(\hat{\omega})(\hat{\mathbf{x}})\right)^{\top} \cdot \operatorname{grad}_{\hat{\mathbf{x}}} \hat{\psi}(\hat{\mathbf{x}}) d \hat{\mathbf{x}}=\int_{\hat{T}}(\hat{\omega}(\hat{\mathbf{x}}))^{\top} \cdot \operatorname{grad}_{\hat{\mathbf{x}}} \hat{\psi}(\hat{\mathbf{x}}) d \hat{\mathbf{x}} \quad \forall \hat{\psi} \in \mathcal{P}_{\tilde{r}(\hat{T})}(\hat{T}) / \mathbb{R} .
$$


Notice that $\stackrel{\circ}{\mathcal{P}}_{\tilde{r}+2} \Lambda^{0}\left(\hat{T} ; \mathbb{R}^{2}\right)=\hat{\chi}(\hat{\mathbf{x}})\left[\mathcal{P}_{\tilde{r}(\hat{T})-1}(\hat{T})\right]^{2}$ where $\hat{\chi}$ is a third order polynomial vanishing along boundary $\partial \hat{T}$ and positive in the interior of $\hat{T}$ (a "bubble"). And notice that

$$
\begin{aligned}
& \left\{\mathbf{h}_{\tilde{r}, 1}(\hat{\mathbf{x}}, 1), \cdots, \mathbf{h}_{\tilde{r}, k_{\tilde{r}}}(\hat{\mathbf{x}}, 1)\right\} \oplus \operatorname{grad}_{\hat{\mathbf{x}}} \mathcal{P}_{\tilde{r}(\hat{T})}(\hat{T}) / \mathbb{R} \\
= & \left\{\mathbf{g}_{\tilde{r}, 1}(\hat{\mathbf{x}}), \cdots, \mathbf{g}_{\tilde{r}, k_{\tilde{r}}}(\hat{\mathbf{x}})\right\} \oplus \operatorname{grad}_{\hat{\mathbf{x}}} \mathcal{P}_{\tilde{r}(\hat{T})}(\hat{T})=\left[\mathcal{P}_{\tilde{r}(\hat{T})-1}(\hat{T})\right]^{2} .
\end{aligned}
$$

Consequently, $\operatorname{det}(C(1, r)) \neq 0$. Since $\operatorname{det}(C(t, r))$ is a polynomial in $t$, by the fundamental theorem of algebra argument again, $\operatorname{det}(C(1, r))$ vanishes at a finite number of roots only.

We can conclude thus that operator $C_{T, t}: H^{1}\left(\hat{T} ; \mathbb{R}^{2}\right) \longrightarrow \mathcal{P}_{\tilde{r}+2} \Lambda^{0}\left(\hat{T} ; \mathbb{R}^{2}\right)$ is welldefined for almost all $t \in[0,1]$. Notice that the number of roots is independent of the choice of the bases, and depends upon $\tilde{r}(\hat{T})$ only.

Definition 6.21. For any $T \in \mathcal{T}_{h}$, we define a linear operator $W_{T, t}: H^{1}\left(T ; \mathbb{R}^{2}\right) \longrightarrow$ $\mathcal{P}_{\tilde{r}+2} \Lambda^{0}\left(T ; \mathbb{R}^{2}\right)$ by the following relations

$$
\begin{gathered}
\int_{T} \operatorname{div}\left(W_{T, t} \omega-\omega\right)(\mathbf{x}) \hat{\psi}(\hat{\mathbf{x}}(\mathbf{x})) d \mathbf{x}=0 \quad \forall \hat{\psi} \in \mathcal{P}_{\tilde{r}(T)}(\hat{T}) / \mathbb{R} \\
\int_{T}\left(W_{T, t} \omega(\mathbf{x})-\omega(\mathbf{x})\right)^{\top} D G_{T}(\hat{\mathbf{x}}(\mathbf{x}))^{-\top} \hat{\mathbf{h}}_{i}(\hat{\mathbf{x}}(\mathbf{x}), t) d \mathbf{x}=0 \quad 1 \leq i \leq k_{\tilde{r}}
\end{gathered}
$$

$\int_{[0,1]}\left[\left(W_{T, t} \omega-\omega\right)\left(\mathbf{x}_{e}(s)\right) \cdot \mathbf{n}\left(\mathbf{x}_{e}(s)\right)\right] \hat{\eta}(s)\left\|\dot{\mathbf{x}}_{e}(s)\right\| d s=0 \quad \forall \hat{\eta} \in \mathcal{P}_{\tilde{r}(e)}([0,1]), \forall e \in \triangle_{1}(T)$

$\int_{[0,1]}\left[\left(W_{T, t} \omega-\omega\right)\left(\mathbf{x}_{e}(s)\right) \cdot \mathbf{t}\left(\mathbf{x}_{e}(s)\right)\right] \hat{\eta}(s)\left\|\dot{\mathbf{x}}_{e}(s)\right\| d s=0 \quad \forall \hat{\eta} \in \mathcal{P}_{\tilde{r}(e)}([0,1]), \forall e \in \triangle_{1}(T)$

$$
W_{T, t} \omega=0 \text { at all vertices of } T
$$

Here $\mathbf{n}, \mathbf{t}$ denote the normal and tangent unit vectors along $\partial T$.

Theorem 6.22. For any $t \in[0,1]$ such that $C_{T, t}: H^{1}\left(\hat{T} ; \mathbb{R}^{2}\right) \longrightarrow \mathcal{P}_{\tilde{r}+2} \Lambda^{0}\left(\hat{T} ; \mathbb{R}^{2}\right)$ is well-defined, there exist $\delta>0$ and $C>0$ such that, for any $h<\delta, T \in \mathcal{T}_{h}$, operator $W_{T, t}: H^{1}\left(T ; \mathbb{R}^{2}\right) \longrightarrow \mathcal{P}_{\tilde{r}+2} \Lambda^{0}\left(T ; \mathbb{R}^{2}\right)$ is well-defined as well and,

$$
\left\|\operatorname{curl} W_{T, t} \omega\right\|_{L^{2}(T)} \leq C\left(\tilde{h}_{T}^{-1}\|\omega\|_{L^{2}(T)}+\|\omega\|_{H^{1}(T)}\right) \quad \forall \omega \in H^{1}\left(T ; \mathbb{R}^{2}\right)
$$

For affine meshes, $W_{T, t}$ is well-defined and the above inequality holds for any $h>0$.

Proof. For any $h>0$ and any $T \in \mathcal{T}_{h}$, we define a linear isomorphism $A_{T}$ from $H^{1}\left(\hat{T} ; \mathbb{R}^{2}\right)$ to $H^{1}\left(T ; \mathbb{R}^{2}\right)$ by $\left(A_{T} \hat{\omega}\right)(\mathbf{x}(\hat{\mathbf{x}}))=\frac{B_{T} \hat{\omega}(\hat{\mathbf{x}})}{\operatorname{det}\left(B_{T}\right)}$. It is easy to see that $A_{T}$ is a linear isomorphism from $\mathcal{P}_{\tilde{r}+2} \Lambda^{0}\left(\hat{T} ; \mathbb{R}^{2}\right)$ to $\mathcal{P}_{\tilde{r}+2} \Lambda^{0}\left(T ; \mathbb{R}^{2}\right)$.

We define an operator $E_{T, t}: H^{1}\left(\hat{T} ; \mathbb{R}^{2}\right) \longrightarrow \mathcal{P}_{\tilde{r}+2} \Lambda^{0}\left(\hat{T} ; \mathbb{R}^{2}\right)$ by $W_{T, t}\left(A_{T} \hat{\omega}\right)=$ $A_{T}\left(E_{T, t} \hat{\omega}\right)$. Obviously, $W_{T, t}$ is well-defined if and only if $E_{T, t}$ is well-defined. We denote by $\left\{\hat{\xi}_{1}, \cdots, \hat{\xi}_{l_{\tilde{r}}}\right\}$ a particular basis of $\mathcal{P}_{\tilde{r}+2} \Lambda^{0}\left(\hat{T} ; \mathbb{R}^{2}\right)$.

According to Lemma 3.15 and Lemma 3.16, it is sufficient to show that there exist $\delta>0$ and $C_{1}>0$ such that, for any $h<\delta$ and $T \in \mathcal{T}_{h}, E_{T, t}$ is well-defined, and $\left\|\left(z_{1}, \cdots, z_{l_{\tilde{r}}}\right)\right\| \leq C_{1}\|\hat{\omega}\|_{H^{1}(\hat{T})}$ for any $\hat{\omega}$. Here $\sum_{k=1}^{l_{\tilde{r}}} z_{k} \hat{\xi}_{k}=E_{T, t} \hat{\omega}$. 
According to the definition of $W_{T, t}, E_{T, t}$ can be defined by relations

$$
\int_{T} \operatorname{div}\left(A_{T} E_{T, t} \hat{\omega}-A_{T} \hat{\omega}\right)(\mathbf{x}) \hat{\psi}(\hat{\mathbf{x}}(\mathbf{x})) d \mathbf{x}=0 \quad \forall \hat{\psi} \in \mathcal{P}_{\tilde{r}(T)}(\hat{T}) / \mathbb{R}
$$

$$
\int_{T}\left(\left(A_{T} E_{T, t} \hat{\omega}\right)(\mathbf{x})-\left(A_{T} \hat{\omega}\right)(\mathbf{x})\right)^{\top} D G_{T}(\hat{\mathbf{x}}(\mathbf{x}))^{-\top} \hat{\mathbf{h}}_{i}(\hat{\mathbf{x}}(\mathbf{x}), t) d \mathbf{x}=0 \quad 1 \leq i \leq k_{\tilde{r}}
$$

$\int_{[0,1]}\left[\left(A_{T} E_{T, t} \hat{\omega}-A_{T} \hat{\omega}\right)\left(\mathbf{x}_{e}(s)\right) \cdot \mathbf{n}\left(\mathbf{x}_{e}(s)\right)\right] \hat{\eta}(s)\left\|\dot{\mathbf{x}}_{e}(s)\right\| d s=0 \quad \forall \hat{\eta} \in \mathcal{P}_{\tilde{r}(e)}([0,1]), \forall e \in \triangle_{1}(T)$ $(6.31)$

$\int_{[0,1]}\left[\left(A_{T} E_{T, t} \hat{\omega}-\hat{\omega}\right)\left(\mathbf{x}_{e}(s)\right) \cdot \mathbf{t}\left(\mathbf{x}_{e}(s)\right)\right] \hat{\eta}(s)\left\|\dot{\mathbf{x}}_{e}(s)\right\| d s=0 \quad \forall \hat{\eta} \in \mathcal{P}_{\tilde{r}(e)}([0,1]), \forall e \in \triangle_{1}(T)$

$$
E_{T, t} \hat{\omega}=0 \text { at all vertices of } \hat{T}
$$

Denote:

$\left[\begin{array}{ll}b_{11} & b_{12} \\ b_{21} & b_{22}\end{array}\right]=B_{T}, J=\operatorname{det}\left(B_{T}\right),\left[\begin{array}{c}\hat{u}_{1} \\ \hat{u}_{2}\end{array}\right]=E_{T, t} \hat{\omega},\left[\begin{array}{c}\hat{w}_{1} \\ \hat{w}_{2}\end{array}\right]=\hat{\omega}, \hat{u}_{i, j}=\frac{\partial \hat{u}_{i}}{\partial \hat{x}_{j}}, \hat{w}_{i, j}=\frac{\partial \hat{w}_{i}}{\partial \hat{x}_{j}}$.

By pulling back to $\hat{T}, E_{T, t}$ can be defined by relations

$$
\begin{gathered}
\int_{\hat{T}} J^{-1}\left[\left(b_{11}\left(D G_{T}\right)_{22}-b_{21}\left(D G_{T}\right)_{12}\right) \hat{u}_{1,1}+\left(b_{12}\left(D G_{T}\right)_{22}-b_{22}\left(D G_{T}\right)_{12}\right) \hat{u}_{2,1}\right. \\
\left.+\left(b_{21}\left(D G_{T}\right)_{11}-b_{11}\left(D G_{T}\right)_{21}\right) \hat{u}_{1,2}+\left(b_{22}\left(D G_{T}\right)_{11}-b_{12}\left(D G_{T}\right)_{21}\right) \hat{u}_{2,2}\right] \hat{\psi}(\hat{\mathbf{x}}) d \hat{\mathbf{x}} \\
=\int_{\hat{T}} J^{-1}\left[\left(b_{11}\left(D G_{T}\right)_{22}-b_{21}\left(D G_{T}\right)_{12}\right) \hat{w}_{1,1}+\left(b_{12}\left(D G_{T}\right)_{22}-b_{22}\left(D G_{T}\right)_{12}\right) \hat{w}_{2,1}\right. \\
\left.\quad+\left(b_{21}\left(D G_{T}\right)_{11}-b_{11}\left(D G_{T}\right)_{21}\right) \hat{w}_{1,2}+\left(b_{22}\left(D G_{T}\right)_{11}-b_{12}\left(D G_{T}\right)_{21}\right) \hat{w}_{2,2}\right] \hat{\psi}(\hat{\mathbf{x}}) d \hat{\mathbf{x}}, \\
\forall \hat{\psi} \in \mathcal{P}_{\tilde{r}(T)}(\hat{T}) / \mathbb{R} \\
(6.34) \quad \int_{\hat{T}}\left(E_{T, t} \hat{\omega}(\hat{\mathbf{x}})\right)^{\top} B_{T}^{\top} D G_{T}(\hat{\mathbf{x}})^{-\top} \hat{\mathbf{h}}_{i}(\hat{\mathbf{x}}, t) \operatorname{det}\left(B_{T}^{-1} D G_{T}(\hat{\mathbf{x}})\right) d \hat{\mathbf{x}} \\
=\int_{\hat{T}}(\hat{\omega}(\hat{\mathbf{x}}))^{\top} B_{T}^{\top} D G_{T}(\hat{\mathbf{x}})^{-\top} \hat{\mathbf{h}}_{i}(\hat{\mathbf{x}}, t) \operatorname{det}\left(B_{T}^{-1} D G_{T}(\hat{\mathbf{x}})\right) d \hat{\mathbf{x}} \quad 1 \leq i \leq k_{\tilde{r}} \\
\quad \int_{[0,1]}\left[B_{T} E_{T, t} \hat{\omega}(\zeta(s)) \cdot\left(D G_{T}(\zeta(s))\right)^{-\top} \hat{\mathbf{n}}(\zeta(s))\right] \hat{\eta}(s) \operatorname{det}\left(B_{T}^{-1} D G_{T}(\zeta(s)) d s\right. \\
=\int_{[0,1]}\left[B_{T} \hat{\omega}(\zeta(s)) \cdot\left(D G_{T}(\zeta(s))\right)^{-\top} \hat{\mathbf{n}}(\zeta(s))\right] \hat{\eta}(s) \operatorname{det}\left(B_{T}^{-1} D G_{T}(\zeta(s)) d s\right.
\end{gathered}
$$

(6.36) $\forall \hat{\eta} \in \mathcal{P}_{\tilde{r}(e)}([0,1]), \forall e \in \triangle_{1}(T)$

$$
\begin{aligned}
& \int_{[0,1]}\left[B_{T} E_{T, t} \hat{\omega}(\zeta(s)) \cdot\left(D G_{T}(\zeta(s))\right) \dot{\zeta}(s)\right] \hat{\eta}(s) \operatorname{det}\left(B_{T}^{-1}\right) d s \\
= & \int_{[0,1]}\left[B_{T} \hat{\omega}(\zeta(s)) \cdot\left(D G_{T}(\zeta(s))\right) \dot{\zeta}(s)\right] \hat{\eta}(s) \operatorname{det}\left(B_{T}^{-1}\right) d s \quad \forall \hat{\eta} \in \mathcal{P}_{\tilde{r}(e)}([0,1]), \forall e \in \triangle_{1}(T)
\end{aligned}
$$




$$
E_{T, t} \hat{\omega}=0 \text { at all vertices of } \hat{T}
$$

Notice that vector $\dot{\zeta}(s)$ is constant tangent vector along each edge of $\hat{T}$. Set

$$
a=\hat{\mathbf{n}}^{\top} B_{T}^{\top} B_{T} \dot{\zeta}(s) \operatorname{det}\left(B_{T}^{-1}\right), \quad b=\frac{\operatorname{det}\left(B_{T}\right)\|\dot{\zeta}(s)\|}{\dot{\zeta}(s)^{\top} B_{T}^{\top} B_{T} \dot{\zeta}(s)}
$$

Obviously, $b \neq 0$.

Perform now the operation: $b \times(6.37)-a \times(6.35)$. We have,

$$
\begin{array}{r}
\int_{[0,1]}\left[E_{T, t} \hat{\omega}(\zeta(s)) \cdot F_{T}(s)\right] \hat{\eta}(s) d s=\int_{[0,1]}\left[\hat{\omega}(\zeta(s)) \cdot F_{T}(s)\right] \hat{\eta}(s) d s \\
\forall \hat{\eta} \in \mathcal{P}_{\tilde{r}(e)}([0,1]), \forall e \in \triangle_{1}(T)
\end{array}
$$

where

$$
\begin{aligned}
F_{T}(s) & =\operatorname{det}\left(B_{T}^{-1}\right)\left[B_{T}^{\top}\left(D G_{T}(\zeta(s))\right) \dot{\zeta}(s)\right. \\
& \left.\left.-\operatorname{det}\left(B_{T}^{-1} D G_{T}(\zeta(s))\right) B_{T}^{\top}\left(D G_{T}(\zeta(s))\right)^{-\top}\left(\hat{\mathbf{n}}^{\top} B_{T}^{T} B_{T} \dot{\zeta}(s)\right) \hat{\mathbf{n}}\right)\right] \frac{\operatorname{det}\left(B_{T}\right)\|\dot{\zeta}(s)\|}{\dot{\zeta}(s)^{\top} B_{T}^{\top} B_{T} \dot{\zeta}(s)}
\end{aligned}
$$

Then the definition of operator $E_{T, t}$ can be rewritten by using conditions (6.33),(6.34), (6.35), (6.39), and (6.38).

Using the fact that $\hat{\mathbf{n}} \perp \dot{\zeta}(s)$, Lemmas 3.7,3.8,3.9, and the assumption that $\left(\mathcal{T}_{h}\right)_{h}$ is regular, we obtain

$\lim _{h \rightarrow 0} \sup _{T \in \mathcal{T}_{h}} \sup _{e \in \triangle_{1}(T)} \sup _{s \in[0,1]}\left\|F_{T}(s) \cdot \frac{\dot{\zeta}(s)}{\|\dot{\zeta}(s)\|}-1\right\|=\lim _{h \rightarrow 0} \sup _{T \in \mathcal{T}_{h}} \sup _{e \in \Delta_{1}(T)} \sup _{s \in[0,1]}\left\|F_{T}(s) \cdot \hat{\mathbf{n}}\right\|=0$

Consequently,

$$
\lim _{h \rightarrow 0} \sup _{T \in \mathcal{T}_{h}} \sup _{e \in \triangle_{1}(T)} \sup _{s \in[0,1]}\left\|F_{T}(s)-\hat{\mathbf{t}}\right\|=0
$$

We denote now by $E(T, t, \tilde{r})$ the matrix corresponding to the left-hand side of conditions $(6.33),(6.34),(6.35),(6.39),(6.38)$, a particular basis of $\mathcal{P}_{\tilde{r}+2} \Lambda^{0}\left(\hat{T} ; \mathbb{R}^{2}\right)$ (the solution space), some basis of $\mathcal{P}_{\tilde{r}(T)}(\hat{T}) / \mathbb{R}$, and some basis of $\mathcal{P}_{\tilde{r}(e)}([0,1])$ for each $e \in \triangle_{1}(T)$. We denote by $\left\{\hat{\xi}_{1}, \cdots, \hat{\xi}_{l \tilde{r}}\right\}$ a basis for $\mathcal{P}_{\tilde{r}+2} \Lambda^{0}\left(\hat{T} ; \mathbb{R}^{2}\right)$. Finally, we denote by $C(T, t, \tilde{r})$ the matrix corresponding to the left-hand side of conditions (6.15), (6.16),(6.17), and (6.18), and the same bases as above.

Using the fact that

$$
\lim _{h \rightarrow 0} \sup _{T \in \mathcal{T}_{h}} \sup _{e \in \triangle_{1}(T)} \sup _{s \in[0,1]}\left\|F_{T}(s)-\hat{\mathbf{t}}\right\|=0
$$

and Lemmas 3.7,3.8, 3.9, we conclude that, for any $t \in[0,1]$,

$$
\lim _{h \rightarrow 0} \sup _{T \in \mathcal{T}_{h}}\|E(T, t, \tilde{r})-C(T, t, \tilde{r})\|=0
$$

Then, for any given $t \in[0,1]$, and any given $\tilde{r}$ with non-singular $C(T, t, \tilde{r})$, the matrix $E(T, t, \tilde{r})$ is non-singular for any $T \in \mathcal{T}_{h}$ when $h>0$ small enough. Notice that the right-hand sides of conditions (6.33), (6.34),(6.35),(6.39), and (6.38) are continuous linear functionals of $\hat{\omega} \in H^{1}\left(\hat{T} ; \mathbb{R}^{2}\right)$. We can conclude thus that the operator $W_{T, t}$ is well-defined for any $T \in \mathcal{T}_{h}$ with small enough $h$.

Since for any $t \in[0,1], \lim _{h \rightarrow 0} \sup _{T \in \mathcal{T}_{h}}\|E(T, t, \tilde{r})-C(T, t, \tilde{r})\|=0$, and the matrix $C(T, t, \tilde{r})$ depends only on $t, \tilde{r}$, we can conclude that there exists $C_{1}>0$ 
such that when $h>0$ small enough, then $\left\|\left(z_{1}, \cdots, z_{l_{\tilde{r}}}\right)\right\| \leq C_{1}\|\hat{\omega}\|_{H^{1}(\hat{T})}$ for any $T \in \mathcal{T}_{h}$. Here $\sum_{k=1}^{l \tilde{r}} z_{k} \hat{\xi}_{k}=E_{T, t} \hat{\omega}$.

Finally, it is easy to see that the operator $W_{T, t}$ will be well-defined and the inequality in the statement of this theorem holds for any $h>0$ for affine meshes. This finishes the proof.

6.3. Projection operators on the whole mesh. According to Lemma 6.17, Theorem 6.18, Lemma 6.20, and Theorem 6.22, there exist $\delta>0$ and $\left\{t_{1}, t_{2}, \cdots\right\} \subset$ $\mathbb{Z}_{+}$such that, for any $h<\delta$ and $T \in \mathcal{T}_{h}, \Pi_{\tilde{r}+1, T, t_{\tilde{r}(T)}}^{1,-}$ and $W_{T, t_{\tilde{r}(T)}}$ are well-defined linear operators. From now on, we rename $\prod_{\tilde{r}+1, T, t_{\tilde{r}(T)}}^{1,-}$ by $\prod_{\tilde{r}+1, T}^{1,-}$, and $W_{T, t_{\tilde{r}(T)}}$ by $W_{T}$.

Definition 6.23. We define the following global interpolation operators,

$$
\begin{gathered}
\Pi_{\tilde{r}, \mathcal{T}_{h}}^{2}: L^{2}(\Omega) \longrightarrow \mathcal{P}_{\tilde{r}} \Lambda^{2}\left(\mathcal{T}_{h}\right),\left.\quad\left(\Pi_{\tilde{r}, \mathcal{T}_{h}}^{2} u\right)\right|_{T}=\Pi_{\tilde{r}, T}^{2}\left(\left.u\right|_{T}\right) \\
\tilde{\Pi}_{\tilde{r}, \mathcal{T}_{h}}^{2}: L^{2}\left(\Omega ; \mathbb{R}^{2}\right) \longrightarrow \mathcal{P}_{\tilde{r}} \Lambda^{2}\left(\mathcal{T}_{h} ; \mathbb{R}^{2}\right),\left.\quad\left(\tilde{\Pi}_{\tilde{r}, \mathcal{T}_{h}}^{2}\left(u_{1}, u_{2}\right)^{\top}\right)\right|_{T}=\left(\Pi_{\tilde{r}, T}^{2}\left(\left.u_{1}\right|_{T}\right), \Pi_{\tilde{r}, T}^{2}\left(\left.u_{2}\right|_{T}\right)\right)^{\top} \\
\Pi_{\tilde{r}+1, \mathcal{T}_{h}}^{1,-}: H^{1}\left(\Omega ; \mathbb{R}^{2}\right) \longrightarrow \mathcal{P}_{\tilde{r}+1}^{-1} \Lambda^{1}\left(\mathcal{T}_{h}\right),\left.\quad\left(\Pi_{\tilde{r}+1, \mathcal{T}_{h}}^{1,-} \omega\right)\right|_{T}=\Pi_{\tilde{r}+1, T}^{1,-}\left(\left.\omega\right|_{T}\right) \\
\tilde{\Pi}_{\tilde{r}+1, \mathcal{T}_{h}}^{1}: H^{1}(\Omega ; \mathbb{M}) \longrightarrow \mathcal{P}_{\tilde{r}+1} \Lambda^{1}\left(\mathcal{T}_{h} ; \mathbb{R}^{2}\right),\left.\quad\left(\tilde{\Pi}_{\tilde{r}+1, \mathcal{T}_{h}}^{1} \sigma\right)\right|_{T}=\left[\begin{array}{cc}
\tau_{11} & \tau_{12} \\
\tau_{21} & \tau_{22}
\end{array}\right]
\end{gathered}
$$

where

$$
\begin{gathered}
{\left[\begin{array}{l}
\tau_{11} \\
\tau_{12}
\end{array}\right]=} \\
\prod_{\tilde{r}+1, \mathcal{T}_{h}}^{1}\left[\begin{array}{l}
\left.\sigma_{11}\right|_{T} \\
\left.\sigma_{12}\right|_{T}
\end{array}\right], \quad\left[\begin{array}{l}
\tau_{21} \\
\tau_{22}
\end{array}\right]=\Pi_{\tilde{r}+1, \mathcal{T}_{h}}^{1}\left[\begin{array}{l}
\left.\sigma_{21}\right|_{T} \\
\left.\sigma_{22}\right|_{T}
\end{array}\right], \quad \sigma=\left[\begin{array}{ll}
\sigma_{11} & \sigma_{12} \\
\sigma_{21} & \sigma_{22}
\end{array}\right] \\
W_{\mathcal{T}_{h}}: H^{1}\left(\Omega ; \mathbb{R}^{2}\right) \longrightarrow \mathcal{P}_{\tilde{r}+2} \Lambda^{0}\left(\mathcal{T}_{h} ; \mathbb{R}^{2}\right),\left.\quad\left(W_{\mathcal{T}_{h}} \omega\right)\right|_{T}=W_{T}\left(\left.\omega\right|_{T}\right)
\end{gathered}
$$

for all $T \in \mathcal{T}_{h}$.

Remark 6.24. Since $\left(\mathcal{T}_{h}\right)_{h}$ is $C^{0}$-compatible, operators $\Pi_{\tilde{r}+1, \mathcal{T}_{h}}^{1,-}, \tilde{\Pi}_{\tilde{r}+1, \mathcal{T}_{h}}^{1}$ and $W_{\mathcal{T}_{h}}$ are well-defined.

Theorem 6.25. For any $\varepsilon>0$, there exists $\delta>0$ such that, for any $h<\delta$,

$$
\left\|\Pi_{\tilde{r}, \mathcal{T}_{h}}^{2} u-P_{\tilde{r}, \mathcal{T}_{h}} u\right\|_{L^{2}(\Omega)} \leq \varepsilon\|u\|_{L^{2}(\Omega)} \quad \forall u \in L^{2}(\Omega)
$$

Here $P_{\tilde{r}, \mathcal{T}_{h}}$ is the standard $L^{2}$-projection onto $\mathcal{P}_{\tilde{r}} \Lambda^{2}\left(\mathcal{T}_{h}\right)$.

Proof. This is an immediate result of Lemma 6.5.

Theorem 6.26. There exist $\delta>0$ and $C>0$ such that, for any $h<\delta$, we have

$$
\left\|\tilde{\Pi}_{\tilde{r}+1, \mathcal{T}_{h}}^{1} \sigma\right\|_{L^{2}(\Omega)} \leq C\|\sigma\|_{H^{1}(\Omega)}, \quad\left\|\Pi_{\tilde{r}+1, \mathcal{T}_{h}}^{1,-} \omega\right\|_{L^{2}(\Omega)} \leq C\|\omega\|_{H^{1}(\Omega)},
$$

for any $\sigma \in H^{1}(\Omega ; \mathbb{M})$ and $\omega \in H^{1}\left(\Omega ; \mathbb{R}^{2}\right)$. For affine meshes, the inequalities above hold for any $h>0$. Moreover,

$$
\operatorname{div} \tilde{\Pi}_{\tilde{r}+1, \mathcal{T}_{h}}^{1} \sigma=\tilde{\Pi}_{\tilde{r}, \mathcal{T}_{h}}^{2} \operatorname{div\sigma }, \quad \operatorname{div} \Pi_{\tilde{r}+1, \mathcal{T}_{h}}^{1,-} \omega=\Pi_{\tilde{r}, \mathcal{T}_{h}}^{2} \operatorname{div} \omega
$$

Proof. This is an immediate result of Lemma 6.11 and Theorem 6.18.

Definition 6.27. Let $R_{h}$ denote the generalized Clement interpolant operator from Theorem 5.1 in [7], mapping $H^{1}\left(\Omega ; \mathbb{R}^{2}\right)$ into $\mathcal{P}_{1} \Lambda^{0}\left(\mathcal{T}_{h} ; \mathbb{R}^{2}\right)$. We define

$$
\tilde{W}_{h}=W_{h}\left(I-R_{h}\right)+R_{h}
$$


Theorem 6.28. There exist $\delta>0$ and $C>0$ such that, for any $h<\delta$,

$$
\left\|\operatorname{curl} \tilde{W}_{h} \omega\right\|_{L^{2}(\Omega)} \leq C\|\omega\|_{H^{1}(\Omega)} \quad \forall \omega \in H^{1}\left(\Omega ; \mathbb{R}^{2}\right)
$$

For affine meshes, the inequality holds for any $h>0$. Operator $\tilde{W}_{h}$ maps $H^{1}\left(\Omega ; \mathbb{R}^{2}\right)$ into $\mathcal{P}_{\tilde{r}+2} \Lambda^{0}\left(T ; \mathbb{R}^{2}\right)$ and satisfies the condition

$$
\Pi_{\tilde{r}+1, \mathcal{T}_{h}}^{1,-} \omega=\Pi_{\tilde{r}+1, \mathcal{T}_{h}}^{1,-} \tilde{W}_{h} \omega \quad \forall \omega \in H^{1}\left(\Omega ; \mathbb{R}^{2}\right)
$$

Proof. We utilize Example 2 from [7] with uniform order equal 1 to construct operator $R_{h}$. Since $\left(\mathcal{T}_{h}\right)_{h}$ is $C^{0}$-compatible, and $c_{h} \rightarrow 0$ as $h \rightarrow 0$, operator $R_{h}$ maps $H^{1}\left(\Omega ; \mathbb{R}^{2}\right)$ into $\mathcal{P}_{1} \Lambda^{0}\left(T ; \mathbb{R}^{2}\right) \subset \mathcal{P}_{\tilde{r}+2} \Lambda^{0}\left(T ; \mathbb{R}^{2}\right)$. According to Theorem 5.1 from [7], there exists a constant $c>0$ such that, for sufficiently small $h$, and any $T \in \mathcal{T}_{h}$,

(6.40) $\left\|\omega-R_{h} \omega\right\|_{L^{2}(T)}+\tilde{h}_{T}\left\|\omega-R_{h} \omega\right\|_{H^{1}(T)} \leq c \tilde{h}_{T}\|\omega\|_{H^{1}\left(K_{T}\right)} \quad \forall \omega \in H^{1}\left(\Omega ; \mathbb{R}^{2}\right)$

where $K_{T}$ stands for the patch of elements adjacent to $T, K_{T}=\bigcup_{T^{\prime} \in \mathcal{T}_{h}, T^{\prime} \cap T \neq \emptyset} T^{\prime}$.

As $\left(\mathcal{T}_{h}\right)_{h}$ is regular, $\sup _{h} \sup _{T \in \mathcal{T}_{h}} \#\left\{T^{\prime} \in \mathcal{T}_{h}: T^{\prime} \subset K_{T}\right\}<\infty$. For sufficiently small $h$, we have

$$
\begin{aligned}
\left\|\operatorname{curl} \tilde{W}_{h} \omega\right\|_{L^{2}(\Omega)} & \leq\left\|\operatorname{curl} W_{h}\left(I-R_{h}\right) \omega\right\|_{L^{2}(\Omega)}+\left\|\operatorname{curl} R_{h} \omega\right\|_{L^{2}(\Omega)} \\
& \leq c\left(\tilde{h}_{T}^{-1}\left\|\left(I-R_{h}\right) \omega\right\|_{L^{2}(\Omega)}+\left\|\left(I-R_{h}\right) \omega\right\|_{H^{1}(\Omega)}+\left\|\operatorname{curl} R_{h} \omega\right\|_{L^{2}(\Omega)}\right) \\
& \leq C\|\omega\|_{H^{1}(\Omega)} \quad \forall \omega \in H^{1}\left(\Omega ; \mathbb{R}^{2}\right)
\end{aligned}
$$

The third inequality above holds by Theorem 6.22 and the fourth one by (6.40).

According to the definition of $\Pi_{\tilde{r}+1, \mathcal{T}_{h}}^{1,-}$, and the definition of $W_{h}, \Pi_{\tilde{r}+1, \mathcal{T}_{h}}^{1,-} \omega=$ $\Pi_{\tilde{r}+1, \mathcal{T}_{h}}^{1,-} W_{h} \omega$, for any $\omega \in H^{1}\left(\Omega ; \mathbb{R}^{2}\right)$. Notice that $\left(I-R_{h}\right) \omega \in \mathcal{P}_{1} \Lambda^{0}\left(\mathcal{T}_{h}\right) \subset H^{1}(\Omega)$. This implies

$$
\Pi_{\tilde{r}+1, \mathcal{T}_{h}}^{1,-}\left(I-\tilde{W}_{h}\right) \omega=\Pi_{\tilde{r}+1, \mathcal{T}_{h}}^{1,-}\left(I-W_{h}\right)\left(I-R_{h}\right) \omega=0
$$

So $\Pi_{\tilde{r}+1, \mathcal{T}_{h}}^{1,-} \omega=\Pi_{\tilde{r}+1, \mathcal{T}_{h}}^{1,-} \tilde{W}_{h} \omega$. For affine meshes, $R_{h}$ reduces to the standard Clement operator. It is easy to see then that the discussed inequality holds for any $h>0$.

\section{Stability of the Finite ElEMEnt DiscRetizAtion}

We need the following well-known result from partial differential equations, see e.g. [16].

Lemma 7.1. Let $\Omega$ be a bounded domain in $\mathbb{R}^{2}$ with a Lipschitz boundary. Then, for all $\mu \in L^{2}(\Omega)$, there exists $\eta \in H^{1}\left(\Omega ; \mathbb{R}^{2}\right)$ satisfying div $=\mu$. If, in addition, $\int_{\Omega} \mu=0$, then we can choose $\eta \in \stackrel{\circ}{H}^{1}(\Omega)$.

The main result of this paper is Theorem 7.3 below. Its proof follows the lines of Theorem 9.1 from [14], Theorem 7.1 from [6] and Theorem 11.4 from [5]. The main difference is the use of operator $\tilde{W}_{h}$ in place of the operator $\tilde{\Pi}_{h}^{n-2}$ in [14].

Lemma 7.2. There exist $\delta>0$ and $c>0$ such that, for any $h<\delta$ and any $(\omega, \mu) \in \mathcal{P}_{\tilde{r}} \Lambda^{2}\left(\mathcal{T}_{h}\right) \times \mathcal{P}_{\tilde{r}} \Lambda^{2}\left(\mathcal{T}_{h} ; \mathbb{R}^{2}\right)$, there exists $\sigma \in \mathcal{P}_{\tilde{r}+1} \Lambda^{1}\left(\mathcal{T}_{h} ; \mathbb{R}^{2}\right)$ such that

$$
\operatorname{div} \sigma=\mu, \quad-\Pi_{\tilde{r}, \mathcal{T}_{h}}^{2} S_{1} \sigma=\omega
$$

and

$$
\|\sigma\|_{H(d i v, \Omega)} \leq c\left(\|\mu\|_{L^{2}(\Omega)}+\|\omega\|_{L^{2}(\Omega)}\right)
$$


Here, the constant c depends on $\sup _{h} \sup _{T \in \mathcal{T}_{h}} \tilde{r}(T)$. For affine meshes, the inequality above holds for any $h>0$.

Proof. (1) By Lemma 7.1, we can find $\eta \in H^{1}(\Omega ; \mathbb{M})$ with div $\eta=\mu$ and $\|\eta\|_{H^{1}(\Omega)} \leq$ $c\|\mu\|_{L^{2}(\Omega)}$.

(2) Since $\omega+\Pi_{\tilde{r}, h}^{2} S_{1} \tilde{\Pi}_{\tilde{r}+1, h}^{1} \eta \in L^{2}(\Omega)$, we can apply Lemma 7.1 again to find $\tau \in H^{1}\left(\Omega ; \mathbb{R}^{2}\right)$ with $\operatorname{div} \tau=\omega+\Pi_{\tilde{r}, h}^{2} S_{1} \tilde{\Pi}_{\tilde{r}+1, h}^{1} \eta$ such that

$$
\|\tau\|_{H^{1}(\Omega)} \leq c\left(\|\omega\|_{L^{2}(\Omega)}+\left\|\Pi_{\tilde{r}, h}^{2} S_{1} \tilde{\Pi}_{\tilde{r}+1, h}^{1} \eta\right\|_{L^{2}(\Omega)}\right)
$$

(3) Define $\sigma=\operatorname{curl} \tilde{W}_{h} \tau+\tilde{\Pi}_{\tilde{r}+1, h}^{1} \eta \in \mathcal{P}_{\tilde{r}+1} \Lambda^{1}\left(\mathcal{T}_{h} ; \mathbb{R}^{2}\right)$. According to Theorem $6.28, \tilde{W}_{h}$ is well-defined, for sufficiently small $h . \quad \sigma \in \mathcal{P}_{\tilde{r}+1} \Lambda^{1}\left(\mathcal{T}_{h} ; \mathbb{R}^{2}\right)$ by Lemma 4.11.

(4) From Step (3), Theorem 6.26, Step (1), and the fact that $\tilde{\Pi}_{\tilde{r}, h}^{2} \tilde{\Pi}_{\tilde{r}, h}^{2}=\tilde{\Pi}_{\tilde{r}, h}^{2}$, we obtain

$$
\operatorname{div} \sigma=\operatorname{div} \tilde{\Pi}_{\tilde{r}+1, h}^{1} \eta=\tilde{\Pi}_{\tilde{r}, h}^{2} \operatorname{div} \eta=\tilde{\Pi}_{\tilde{r}, h}^{2} \mu=\mu .
$$

(5) Notice that $\operatorname{div} \omega=-S_{1} \operatorname{curl} \omega$, for any $\omega \in H^{1}\left(\Omega ; \mathbb{R}^{2}\right)$. Here operator $S_{1}$ is defined in (1.10). Then $\Pi_{\tilde{r}, h}^{2} \operatorname{div} \Pi_{\tilde{r}+1, h}^{1,-} \omega=-\Pi_{\tilde{r}, h}^{2} S_{1} \operatorname{curl} \omega$, for any $\omega \in H^{1}\left(\Omega ; \mathbb{R}^{2}\right)$.

(6) Also from Step (3), $-\Pi_{\tilde{r}, h}^{2} S_{1} \sigma=-\Pi_{\tilde{r}, h}^{2} S_{1} \operatorname{curl} \tilde{W}_{h} \tau-\Pi_{\tilde{r}, h}^{2} S_{1} \tilde{\Pi}_{\tilde{r}+1, h}^{1} \eta$. Applying, in order, Step (5), Theorem 6.28, Theorem 6.26, Step (2), and the fact that $\Pi_{\tilde{r}, h}^{2} \Pi_{\tilde{r}, h}^{2}=\Pi_{\tilde{r}, h}^{2}$, we have

$$
\begin{aligned}
-\Pi_{\tilde{r}, h}^{2} S_{1} \operatorname{curl} \tilde{W}_{h} \tau & =\Pi_{\tilde{r}, h}^{2} \operatorname{div} \Pi_{\tilde{r}+1, h}^{1,-} \tilde{W}_{h} \tau=\Pi_{\tilde{r}, h}^{2} \operatorname{div} \Pi_{\tilde{r}+1, h}^{1,-} \tau=\Pi_{\tilde{r}, h}^{2} \operatorname{div} \tau \\
& =\Pi_{\tilde{r}, h}^{2}\left(\omega+\Pi_{\tilde{r}, h}^{2} S_{1} \tilde{\Pi}_{\tilde{r}+1, h}^{1} \eta\right)=\omega+\Pi_{\tilde{r}, h}^{2} S_{1} \tilde{\Pi}_{\tilde{r}+1, h}^{1} \eta
\end{aligned}
$$

Combining, we have $-\Pi_{\tilde{r}, h}^{2} S_{1} \sigma=\omega$.

(7) Finally, we prove the norm bound. From Theorem 6.25, the boundedness of $S_{1}$ in $L^{2}$, Theorem 6.26, and Step (1), for sufficiently small $h$, we obtain

$$
\begin{aligned}
\left\|\Pi_{\tilde{r}, h}^{2} S_{1} \tilde{\Pi}_{\tilde{r}+1, h}^{1} \eta\right\|_{L^{2}(\Omega)} & \leq c\left\|S_{1} \tilde{\Pi}_{\tilde{r}+1, h}^{1} \eta\right\|_{L^{2}(\Omega)} \leq c\left\|\tilde{\Pi}_{\tilde{r}+1, h}^{1} \eta\right\|_{L^{2}(\Omega)} \\
& \leq c\|\eta\|_{H^{1}(\Omega)} \leq c\|\mu\|_{L^{2}(\Omega)} .
\end{aligned}
$$

Combining the result with the bound in Step (2), we get $\|\tau\|_{H^{1}(\Omega)} \leq c\left(\|\omega\|_{L^{2}(\Omega)}+\right.$ $\left.\|\mu\|_{L^{2}(\Omega)}\right)$. Theorem 6.28 then yields

$$
\| \operatorname{curl}\left[\tilde{W}_{h} \tau\left\|_{L^{2}(\Omega)} \leq c\right\| \tau \|_{H^{1}(\Omega)} \leq c\left(\|\omega\|_{L^{2}(\Omega)}+\|\mu\|_{L^{2}(\Omega)}\right)\right.
$$

for sufficiently small $h$. From Theorem 6.26 and the bound in Step (1),

$$
\left\|\tilde{\Pi}_{, \tilde{r}+1, h}^{1} \eta\right\|_{L^{2}(\Omega)} \leq c\|\eta\|_{H^{1}(\Omega)} \leq c\|\mu\|_{L^{2}(\Omega)}
$$

for small enough $h$. In view of the definition of $\sigma$, these two last bounds imply that $\|\sigma\|_{L^{2}(\Omega)} \leq c\left(\|\omega\|_{L^{2}(\Omega)}+\|\mu\|_{L^{2}(\Omega)}\right)$, while $\|\operatorname{div} \sigma\|_{L^{2}(\Omega)}=\|\mu\|_{L^{2}(\Omega)}$ by Step (4). It is easy to see that, for affine meshes, all the discussed steps hold for any $h>0$. This finishes the proof.

Theorem 7.3. There exist $\delta>0$ and $c>0$ such that, for solution $(\sigma, u, p)$ of elasticity system (1.8), and corresponding solution $\left(\sigma_{h}, u_{h}, p_{h}\right)$ of discrete system (5.1), we have

$$
\begin{gathered}
\left\|\sigma-\sigma_{h}\right\|_{H(d i v, \Omega)}+\left\|u-u_{h}\right\|_{L^{2}(\Omega)}+\left\|p-p_{h}\right\|_{L^{2}(\Omega)} \\
\leq c \inf \left[\|\sigma-\tau\|_{H(d i v, \Omega)}+\|u-v\|_{L^{2}(\Omega)}+\|p-q\|_{L^{2}(\Omega)}\right],
\end{gathered}
$$


where the infimum is taken over all $\tau \in \mathcal{P}_{\tilde{r}+1} \Lambda^{1}\left(\mathcal{T}_{h}, \mathbb{R}^{2}\right), v \in \mathcal{P}_{\tilde{r}} \Lambda^{2}\left(\mathcal{T}_{h}, \mathbb{R}^{2}\right)$, and $q \in \mathcal{P}_{\tilde{r}} \Lambda^{2}\left(\mathcal{T}_{h}\right)$, for $h<\delta$. For affine meshes, the inequality holds for any $h>0$.

Proof. We need to show that conditions (5.3) and (5.4) are satisfied asymptotically in $h$. Condition (5.3) follows from the fact that, by construction, $\operatorname{div} \mathcal{P}_{\tilde{r}+1} \Lambda^{1}\left(\mathcal{T}_{h}, \mathbb{R}^{2}\right) \subset$ $\mathcal{P}_{\tilde{r}} \Lambda^{2}\left(\mathcal{T}_{h} ; \mathbb{R}^{2}\right)$, and the fact that $A$ is coercive.

We turn now to condition (5.4). According to Lemma 7.2, there exist $\delta>0$ and $c>0$ such that, for $h<\delta$ and $(\omega, \mu) \in \mathcal{P}_{\tilde{r}} \Lambda^{2}\left(\mathcal{T}_{h}\right) \times \mathcal{P}_{\tilde{r}} \Lambda^{2}\left(\mathcal{T}_{h} ; \mathbb{R}^{2}\right)$, there exists $\sigma \in \mathcal{P}_{\tilde{r}+1} \Lambda^{1}\left(\mathcal{T}_{h} ; \mathbb{R}^{2}\right)$ such that $\operatorname{div} \sigma=\mu,-\Pi_{\tilde{r}, \mathcal{T}_{h}}^{2} S_{1} \sigma=\omega$, and $\|\sigma\|_{H(\operatorname{div}, \Omega)} \leq$ $c\left(\|\mu\|_{L^{2}(\Omega)}+\|\omega\|_{L^{2}(\Omega)}\right)$. We have then

$$
\begin{aligned}
\langle\operatorname{div} \sigma, \mu\rangle-\left\langle S_{1} \sigma, \omega\right\rangle & =\langle\operatorname{div} \sigma, \mu\rangle-\left\langle\Pi_{\tilde{r}, \mathcal{T}_{h}}^{2} S_{1} \sigma, \omega\right\rangle+\left\langle\left(\Pi_{\tilde{r}, \mathcal{T}_{h}}^{2}-P_{\tilde{r}, \mathcal{T}_{h}}\right) S_{1} \sigma, \omega\right\rangle \\
& \geq c\|\sigma\|_{H(\operatorname{div}, \Omega)}\left(\|\mu\|_{L^{2}(\Omega)}+\|\omega\|_{L^{2}(\Omega)}\right)+\left\langle\left(\Pi_{\tilde{r}, \mathcal{T}_{h}}^{2}-P_{\tilde{r}, \mathcal{T}_{h}}\right) S_{1} \sigma, \omega\right\rangle .
\end{aligned}
$$

According to Theorem 6.25 , for sufficiently small $h$,

$$
\left|\left\langle\left(\Pi_{\tilde{r}, \mathcal{T}_{h}}^{2}-P_{\tilde{r}, \mathcal{T}_{h}}\right) S_{1} \sigma, \omega\right\rangle\right| \leq \frac{c}{2}\|\sigma\|_{L^{2}(\Omega)}\|\omega\|_{L^{2}(\Omega)}
$$

So, asymptotically in $h$, we have

$$
\langle\operatorname{div} \sigma, \mu\rangle-\left\langle S_{1} \sigma, \omega\right\rangle \geq \frac{c}{2}\|\sigma\|_{H(\operatorname{div}, \Omega)}\left(\|\mu\|_{L^{2}(\Omega)}+\|\omega\|_{L^{2}(\Omega)}\right) .
$$

For affine meshes, $\Pi_{\tilde{r}, \mathcal{T}_{h}}^{2}$ reduces to the standard $L^{2}$-projection. The inequality above holds then for any $h>0$. This finishes the proof.

\section{NUMERICAL EXPERIMENTS}

We continue numerical experiments initiated in [19] where we investigated rates of convergence for uniform $h$-refinements in presence of non-uniform polynomial order $p$, and tried out $p$-adaptivity (with no underlying theory at presence). The experiments confirmed the optimal $h$-convergence rates and indicated the $p$-convergence as well.

Following [19], we consider the L-shape domain, and use a manufactured solution corresponding to the exact solution of the homogeneous equilibrium equations (zero volume forces), and the corresponding unbounded L-shape domain extending to infinity. The manufactured solution is designed in such a way that both $p$ and all stress components have the same singularity at the origin characterized by term $r^{-0.39596}$ where $r$ is the distance to the origin.

Experiments presented below focus on $h$-adaptivity for meshes with uniform order $p$, and the ultimate goal of this research - the $h p$-adaptivity. We investigate both affine and curvilinear meshes. We use the standard "greedy algorithm" for $h$-refinements, and the two-grid $h p$-algorithm for $h p$-refinements, see [12] for details. The $h p$-algorithm is based on the standard Projection Based (PB) interpolation. All convergence plots are displayed on log-log scale, error vs. number of degreesof-freedom (d.o.f.). The error is always measured in terms of percent of the total norm of the solution.

Uniform $h$-refinements on affine meshes. We begin with a verification of stability on uniform meshes. Fig. 2 presents the L-shape domain with an initial mesh of six elements. Fig. 3 displays the actual approximation error compared with the best approximation error for a sequence of uniformly refined meshes of zero order ${ }^{3}$.

\footnotetext{
${ }^{3}$ We always refer to the order of approximation for the displacement.
} 


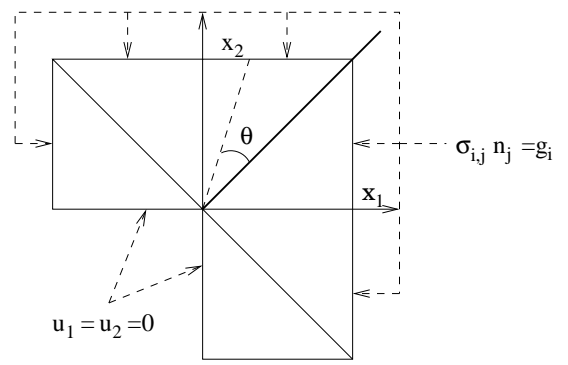

Figure 2. The L shape domain with initial mesh.

As expected, the two curves are parallel to each other. We repeat now the same

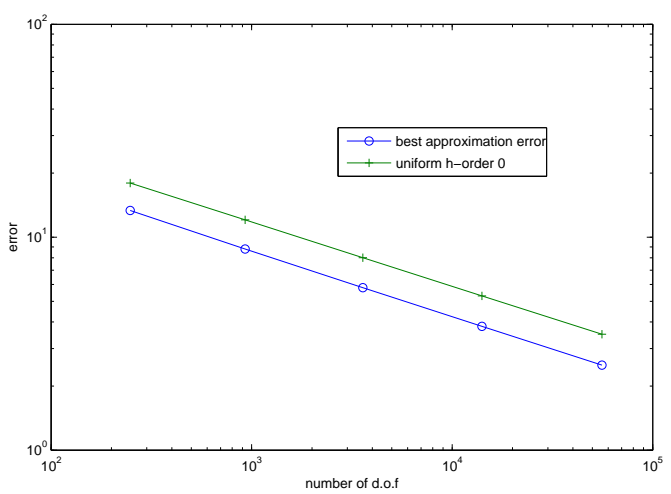

FiguRE 3. Uniform $h$-refinements for $p=0$. FE error vs. best approximation error.

experiment starting with an initial mesh of elements with order varying from zero to four, shown in Fig. 4. The corresponding FE error is compared again with the

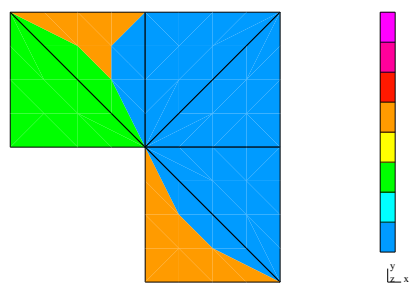

Figure 4. The L shape domain with initial mesh.

best approximation error in Fig. 5. The two lines are again parallel to each other with the slope determined by the lowest order elements in the mesh (same as in the first example). 


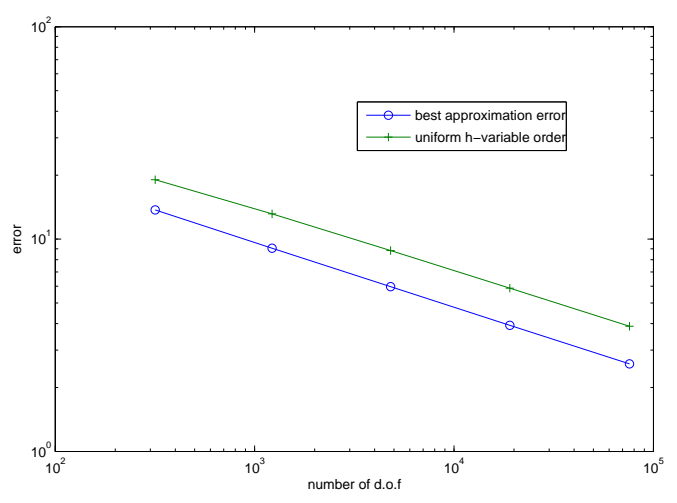

FiguRE 5. Uniform $h$-refinements for a mesh of variable order. FE error vs. best approximation error.

Adaptive $h$ - and $h p$-refinements on affine meshes. We continue now the numerical verification of stability for non-uniform meshes resulting from $h$ - and $h p$-refinements. Unfortunately, we face a slight discrepancy between the presented theory and the numerical experiments as the code is using 1-irregular meshes which we have not accounted for in our theoretical analysis.

Fig. rates presents convergence history for adaptive $h$-refinements for meshes of order $p=0,1,2$ and adaptive $h p$-refinements starting with the mesh of zero order. The approximation error is compared with the best approximation error computed on the same meshes (generated by the adaptive algorithm). Results for $h$-refined meshes of order $p=1,2$, and the $h p$-refinements confirm the stability result. The result for the lowest order elements, though, reflects some loss of stability. The only possible explanation that we have at the moment, is the use of meshes with hanging nodes.

Finally, in Fig. 7, we compare the convergence history for all tested refinements. The $h p$-algorithm delivers clearly an exponential convergence.

Adaptive $h$ - and $h p$-refinements on curvilinear meshes. We use the same manufactured solution on the circular L-shape domain shown in Fig. 8. The six triangles in the initial mesh are parametrized using the transfinite interpolation technique, see [12], p. 201, for details. The FE error is compared again with the best approximation error for a sequence of $h$-adaptive meshes and $p=0,1,2$, in Fig. 9. The results indicate again a slight loss of stability for the elements of lowest order. Finally, in Fig. 10, we compare the convergence history for all tested refinements. The $h p$-adaptivity delivers again the best results but the exponential convergence is questionable. This may indicate that the use of standard ProjectionBased interpolation operator in the reference domain is not optimal.

\section{Conclusions}

We have presented a complete $h$-stability analysis for a generalization of ArnoldFalk-Winther elements to curvilinear meshes of variable order in two space dimensions. The stability analysis for both generalizations: variable order elements, 


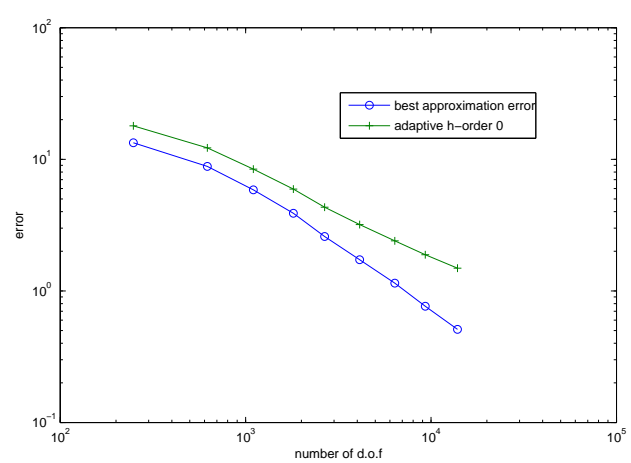

(a) Adaptive $h$-refinements, $p=0$.

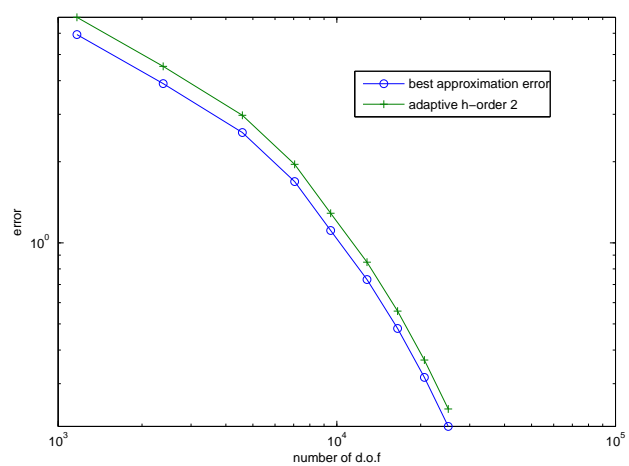

(c) Adaptive $h$-refinements, $p=2$.

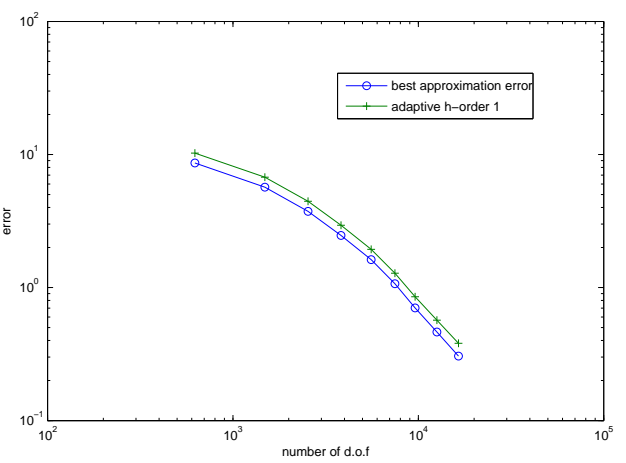

(b) Adaptive $h$-refinements, $p=1$.

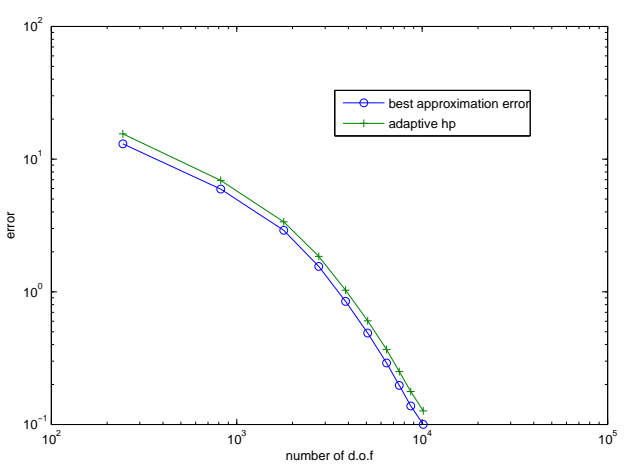

(d) Adaptive $h p$-refinements.

Figure 6. Comparison of FE error for adaptively refined affine meshes with the best approximation error.

and curvilinear elements proved to be rather non-trivial. The case of variable elements has been tackled with a novel logical construction showing the existence of necessary interpolation operators rather than constructing them explicitly. The presented construction departs from operators used by Arnold, Falk and Winther and modifies the Projection-Based (PB) interpolation operators as well.

The analysis of curvilinear meshes for elasticity differs considerably from that for problems involving only grad-curl-div operators. Piola maps transform gradients, curls and divergence in the physical domain into the corresponding gradients, curls and divergence in the reference domain. Consequently, problems involving the grad, curl or div operators only (e.g. Maxwell equations or the mixed formulation for a scalar elliptic problem) can be reformulated in the parametric domain at the expense of introducing material anisotropies reflecting the geometric parametrizations. This is not the case for elasticity where the strain tensor (symmetric part of the displacement gradient) in the physical domain does not transform into the symmetric part of the displacement gradient in the reference domain ${ }^{4}$. Consequently, the analysis for affine meshes cannot be simply reproduced for curvilinear ones,

\footnotetext{
${ }^{4}$ The same problem is encountered in the case of complex stretchings introduced by Perfectly Matched Layers, see e.g. [13].
} 


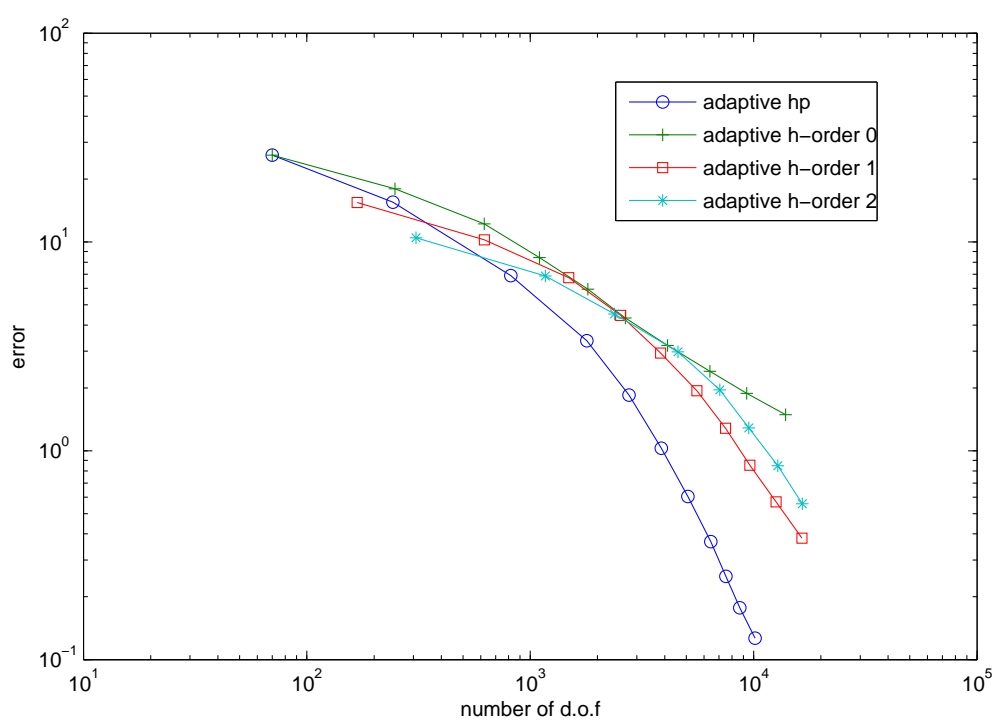

FiguRE 7. Convergence history for adaptive $h$ - and $h p$-refinements on affine meshes.

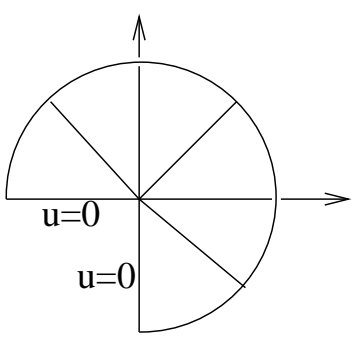

Figure 8. A circular L-shape domain with an initial mesh of six elements.

and new interpolation operators have to be carefully drafted. We have managed to prove only an asymptotic stability for the curvilinear meshes.

Presented numerical results on $h$-adaptivity go beyond our analysis as we use 1-irregular meshes with hanging nodes supported by an existing $h p$ software.

The $h p$-adaptive algorithm used in the experiments is based on the standard PB interpolation which does not reflect the special operators introduced in this paper. This does not seem to affect the case of affine meshes where we clearly observe the exponential convergence, and indicates that the construction of operator $W_{h}$ based on PB interpolation and presented in [19] may still be optimal. However, the experiment for the circular L-shape domain shows that the algorithm based on PB interpolation in the reference domain does not deliver exponential convergence. This seem to confirm the essential difference between the interpolation done in physical and reference domains for elasticity problems.

Finally, the paper presents only a two-dimensional result. We continue working on the 3D case using different ideas and hope to present new results soon. 


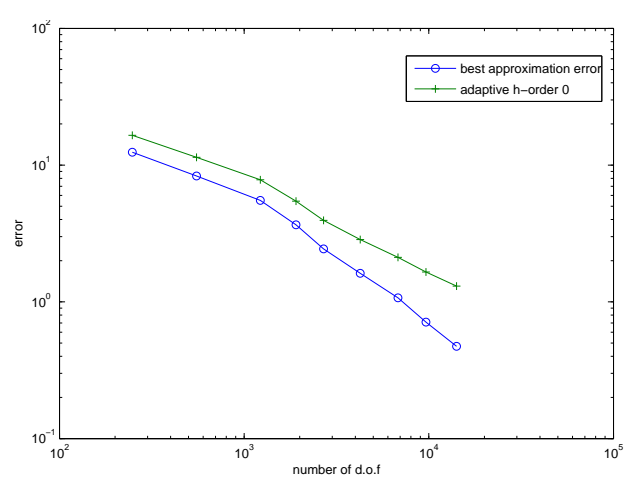

(a) Adaptive $h$-refinements, $p=0$.

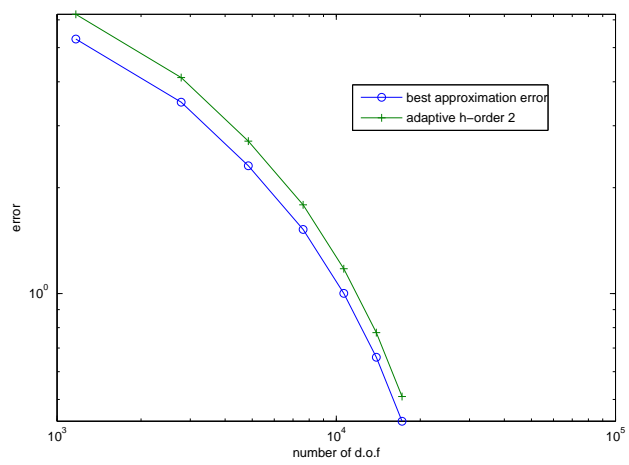

(c) Adaptive $h$-refinements, $p=2$.

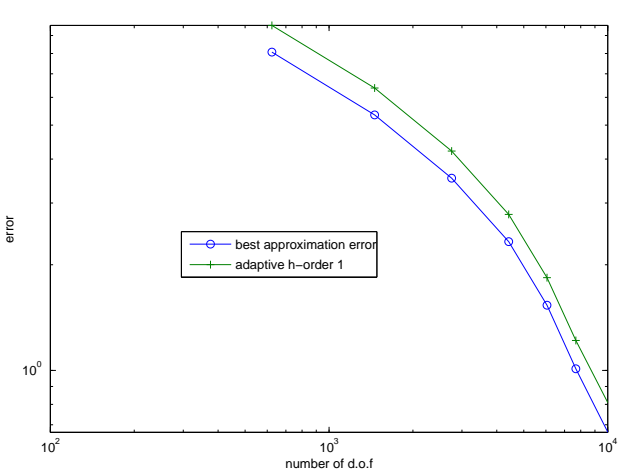

(b) Adaptive $h$-refinements, $p=1$.

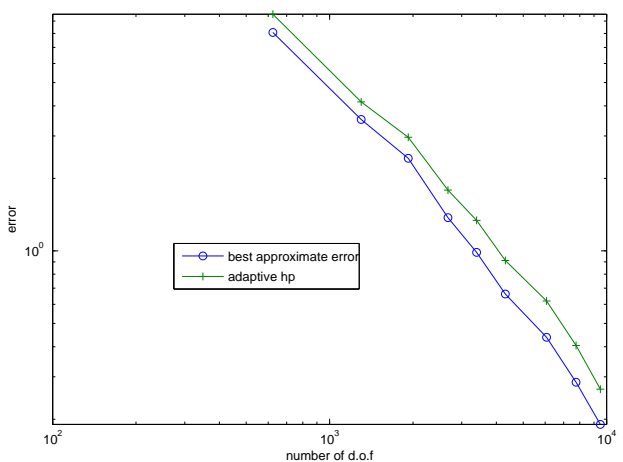

(d) Adaptive $h p$-refinements.

Figure 9. Comparison of FE error for adaptively refined curvilinear meshes with the best approximation error.

\section{REFERENCES}

[1] M. Amara And J. M. Thomas, Equilibrium finite elements for the linear elastic problem, Numer. Math., 33 (1979), pp. 367-383.

[2] D. N. Arnold, G. Awanou, and R. Winther, Finite elements for symmetric tensors in three dimensions, Mathematics of Computation, 77 (2008), pp. 1229-1251.

[3] D. N. Arnold, F. Brezzi, and J. Douglas, Peers: a new mixed finite element for plane elasticity, Japan J. Appl. Math., 1 (1984), pp. 347-367.

[4] D. N. Arnold and R. S. Falk, A new mixed formulation for elasticity, Numer. Math., 53 (1988), pp. 13-30.

[5] D. N. ARnold, R. S. Falk, And R. Winther, Finite element exterior calculus, homological techniques, and applications, Acta Numer., (2006), pp. 1-155.

[6] _ - Mixed finite element methods for linear elasticity with weakly imposed symmetry, Mathematics of Computation, 76 (2007), pp. 1699-1723.

[7] C. Bernardi, Optimal finite-element interpolation on curved domains, SIAM Journal on Numerical Analysis, 26 (1989), pp. 1212-1240.

[8] G. CARey, Computational Grids: Generations, Adaptation and Solution Strategies, CRC Press, 1997.

[9] P. Ciarlet, The Finite Element Methods for Elliptic Problems, SIAM, 2002.

[10] B. Cockburn, J. Gopalakrishnan, and J. Guzman, A new elasticity element made for enforcing weak stress symmetry, Mathematics of Computation, (2009). 


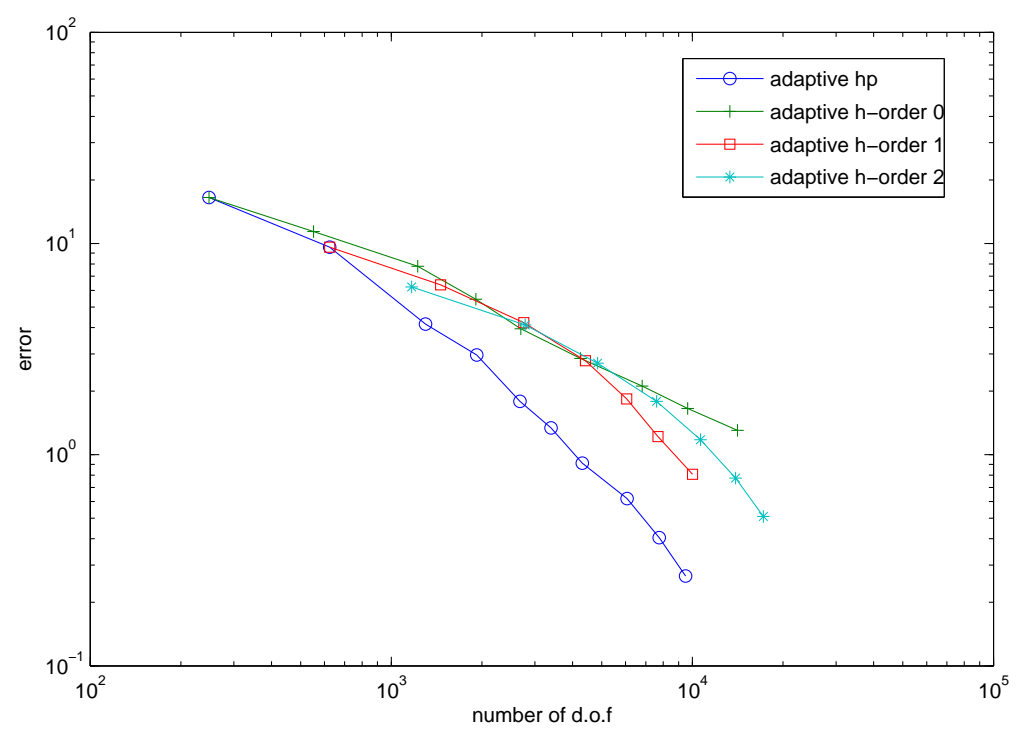

FiguRE 10. Convergence history for adaptive $h$ - and $h p$ refinements on curvilinear meshes.

[11] B. M. F. De Veubeke, Stress function approach, Proc. of the World Congress on Finite Element Methods in Structural Mechanics, 5 (1975), pp. J.1-J.51.

[12] L. Demkowicz, Computing with hp-adaptive finite elements Volume I One and Two Dimensional Elliptic and Maxwell Problems, Chapman Hall CRC, 2006.

[13] L. Demkowicz, J. Kurtz, D. Pardo, M. Paszynski, W. Rachowicz, and A. Zdunek, Computing with hp-adaptive finite elements Volume II Three-Dimensional Elliptic and Maxwell Problems with Applications, Chapman Hall CRC, 2007.

[14] R. S. FALK, Finite element methods for linear elasticity, in Lecture Notes in Mathematics, Springer-Verlag, 2008, pp. 160-194.

[15] M. FARHLOUl AND M. Fortin, Dual hybrid methods for the elasticity and the Stokes problem: a unified approach, Numer. Math., 76 (1997), pp. 419-440.

[16] V. Girault And P. A. Raviart, Finite element methods for Navier-Stokes equations: theory and algorithms, Springer-Verlag, Berlin; New york, 1986.

[17] M. E. Morley, A family of mixed finite elements for linear elasticity, Numer. Math., 55 (1989), pp. 633-666.

[18] J. T. Oden And J. N. Reddy, Variational methods in theoretical mechanics, Springer-Verlag, 1976.

[19] W. Qiu And L. Demkowicz, Mixed hp-finite element method for linear elasticity with weakly imposed symmetry, Comput. Methods Appl. Mech. Engrg., 198 (2009), pp. 3682-3701.

[20] E. Stein And R. Rolfes, Mechanical conditions for stability and optimal convergence of mixed finite elements for linear plane elasticity, Comput. Methods Appl. Mech. Engrg., 84 (1990), pp. 77-95.

[21] R. Stenberg, On the construction of optimal mixed finite element methods for the linear elasticity problem, Numer. Math., 48 (1986), pp. 447-462.

[22] — A family of mixed finite elements for the elasticity problem, Numer. Math., 53 (1988), pp. 513-538.

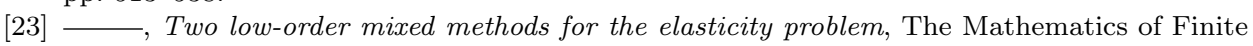
Elements and Applications, VI, (1988), pp. 271-280. 
The Institute for Computational Engineering and Sciences, the University of Texas at Austin, Austin, Texas 78712

E-mail address: qiuw@ices.utexas.edu

The Institute for Computational Engineering and Sciences, the University of Texas at Austin, Austin, Texas 78712

E-mail address: leszek@ices.utexas.edu 